\title{
HAWKES PROCESSES ON LARGE NETWORKS
}

\author{
By Sylvain Delattre, Nicolas Fournier and Marc HofFmann
Université Paris-Diderot, Université Pierre-et-Marie Curie and Université Paris-Dauphine

We generalise the construction of multivariate Hawkes processes to a possibly infinite network of counting processes on a directed graph $\mathbb{G}$. The process is constructed as the solution to a system of Poisson driven stochastic differential equations, for which we prove pathwise existence and uniqueness under some reasonable conditions.

We next investigate how to approximate a standard $N$-dimensional Hawkes process by a simple inhomogeneous Poisson process in the meanfield framework where each pair of individuals interact in the same way, in the limit $N \rightarrow \infty$. In the so-called linear case for the interaction, we further investigate the large time behaviour of the process. We study in particular the stability of the central limit theorem when exchanging the limits $N, T \rightarrow \infty$ and exhibit different possible behaviours.

We finally consider the case $\mathbb{G}=\mathbb{Z}^{d}$ with nearest neighbour interactions. In the linear case, we prove some (large time) laws of large numbers and exhibit different behaviours, reminiscent of the infinite setting. Finally, we study the propagation of a single impulsion started at a given point of $\mathbb{Z}^{d}$ at time 0 . We compute the probability of extinction of such an impulsion and, in some particular cases, we can accurately describe how it propagates to the whole space.

\section{Introduction.}

1.1. Motivation. In several apparently different applied fields, a growing interest has been observed recently for a better understanding of stochastic interactions between multiple entities evolving through time. These include: seismology for modelling earthquake replicas (Helmstetter and Sornette [21], Kagan [27], Ogata [36], Bacry and Muzy [4]), neuroscience for modelling spike trains in brain activity (Grün et al. [17], Okatan et al. [37], Pillow et al. [38], Reynaud et al. [39, 40]), genome analysis (Reynaud-Bouret and Schbath [41]), financial contagion (Ait-Sahalia et al. [1]), high-frequency finance (order arrivals, see Bauwens and Hautsch [6], Hewlett [22], market microstructure see Bacry et al. [2] and market impact see Bacry and Muzy [4, 5]), financial price modelling across scales (Bacry et al. [3], Jaisson and Rosenbaum [25]), social networks interactions (Blundell

Received March 2014; revised November 2014.

MSC2010 subject classifications. 60F05, 60G55, 60G57.

Key words and phrases. Point processes, multivariate Hawkes processes, stochastic differential equations, limit theorems, mean-field approximations, interacting particle systems. 
et al. [7], Simma and Jordan [42], Zhou et al. [46]) and epidemiology like for instance viral diffusion on a network (Hang and Zha [45]), to name but a few. In all these contexts, observations are often represented as events (like spikes or features) associated to agents or nodes on a given network, and that arrive randomly through time but that are not stochastically independent.

In practice, we observe a multivariate counting process $\left(Z_{t}^{1}, \ldots, Z_{t}^{N}\right)_{t \geq 0}$, each component $Z_{t}^{i}$ recording the number of events of the $i$ th component of the system during $[0, t]$, or equivalently the time stamps of the observed events. Under relatively weak general assumptions, a multivariate counting process $\left(Z_{t}^{1}, \ldots, Z_{t}^{N}\right)_{t \geq 0}$ is characterised by its intensity process $\left(\lambda_{t}^{1}, \ldots, \lambda_{t}^{N}\right)_{t \geq 0}$, informally defined by

$$
\operatorname{Pr}\left(Z^{i} \text { has a jump in }[t, t+d t] \mid \mathcal{F}_{t}\right)=\lambda_{t}^{i} d t, \quad i=1, \ldots, N,
$$

where $\mathcal{F}_{t}$ denotes the sigma-field generated by $\left(Z^{i}\right)_{1 \leq i \leq N}$ up to time $t$. For modelling the interactions, a particularly attractive family of multivariate point processes is given by the class of (mutually exciting) Hawkes processes (Hawkes [19], Hawkes and Oakes [20]), with intensity process given by

$$
\lambda_{t}^{i}=h_{i}\left(\sum_{j=1}^{N} \int_{0}^{t-} \varphi_{j i}(t-s) d Z_{s}^{j}\right),
$$

where the causal functions $\varphi_{j i}:[0, \infty) \rightarrow \mathbb{R}$ model how $Z^{j}$ acts on $Z^{i}$ by affecting its intensity process $\lambda^{i}$. The nonnegative functions $h_{i}$ account for some nonlinearity, but if we set $h_{i}(x)=\mu_{i}+x$ with $\mu_{i} \geq 0$, we obtain linear Hawkes processes where $\mu_{i}$ can be interpreted as a baseline Poisson intensity. In the degenerate case $\varphi_{j i}=0$, we actually retrieve standard Poisson processes.

Multivariate Hawkes processes have long been studied in probability theory (see, e.g., the comprehensive textbook of Daley and Vere-Jones [12] and the references therein, Brémaud and Massoulié [9] and Massoulié [32] or the recent results of Zhu [47, 49]). Their statistical inference is relatively well understood, too, from a classical parametric angle (Ogata [34]) together with recent significant advances in nonparametrics (Reynaud-Bouret and Schbath [41], Hansen et al. [18]). However, the frontier is progressively moving to understanding the case of large $N$, when the number of components may become increasingly large or possibly infinite; see Massoulié [32] and Galvez and Löcherbach [15] for some constructions in that direction. This context is potentially of major importance for future developments in the aforementioned applied fields. This is the topic of the present paper.

1.2. Setting. We work on a filtered probability space $\left(\Omega, \mathcal{F},\left(\mathcal{F}_{t}\right)_{t \geq 0}, \operatorname{Pr}\right)$. We say that $\left(X_{t}\right)_{t \geq 0}$ is a counting process if it is nondecreasing, càdlàg, integer-valued (and finite for all times), with all its jumps of height 1 . For $\left(X_{t}\right)_{t \geq 0}$ a $\left(\mathcal{F}_{t}\right)_{t \geq 0^{-}}$ adapted counting process, there is a unique nondecreasing predictable process $\left(\Lambda_{t}\right)_{t \geq 0}$, called compensator of $\left(X_{t}\right)_{t \geq 0}$, such that $\left(X_{t}-\Lambda_{t}\right)_{t \geq 0}$ is a $\left(\mathcal{F}_{t}\right)_{t \geq 0}$-local martingale; see Jacod and Shiryaev [24], Chapter I. 
We consider a countable directed graph $\mathbb{G}=(\mathcal{S}, \mathcal{E})$ with vertices (or nodes) $i \in \mathcal{S}$ and (directed) edges $e \in \mathcal{E}$. We write $e=(j, i) \in \mathcal{E}$ for the oriented edge. We also need to specify the following parameters: a kernel $\boldsymbol{\varphi}=\left(\varphi_{j i},(j, i) \in \mathcal{E}\right)$ with $\varphi_{j i}:[0, \infty) \mapsto \mathbb{R}$, and a nonlinear intensity component $\mathbf{h}=\left(h_{i}, i \in \mathcal{S}\right)$ with $h_{i}: \mathbb{R} \mapsto[0, \infty)$. The natural generalisation of finite-dimensional Hawkes processes is the following.

DEFINITION 1. A Hawkes process with parameters $(\mathbb{G}, \boldsymbol{\varphi}, \mathbf{h})$ is a family of $\left(\mathcal{F}_{t}\right)_{t \geq 0}$-adapted counting processes $\left(Z_{t}^{i}\right)_{i \in \mathcal{S}, t \geq 0}$ such that:

(i) almost surely, for all $i \neq j,\left(Z_{t}^{i}\right)_{t \geq 0}$ and $\left(Z_{t}^{j}\right)_{t \geq 0}$ never jump simultaneously,

(ii) for every $i \in \mathcal{S}$, the compensator $\left(\Lambda_{t}^{i}\right)_{t \geq 0}$ of $\left(Z_{t}^{i}\right)_{t \geq 0}$ has the form $\Lambda_{t}^{i}=$ $\int_{0}^{t} \lambda_{s}^{i} d s$, where the intensity process $\left(\lambda_{t}^{i}\right)_{t \geq 0}$ is given by

$$
\lambda_{t}^{i}=h_{i}\left(\sum_{j \rightarrow i} \int_{0}^{t-} \varphi_{j i}(t-s) d Z_{s}^{j}\right),
$$

with the notation $\sum_{j \rightarrow i}$ for summation over $\{j:(j, i) \in \mathcal{E}\}$.

This model can be seen as a particular case of that introduced by Massoulié [32] who considers more general sets of sites (possibly $\mathbb{R}^{d}$ ) and a larger class of intensities. We say that a Hawkes process is linear when $h_{i}(x)=\mu_{i}+x$ for every $x \in \mathbb{R}, i \in \mathcal{S}$, with $\mu_{i} \geq 0$ and when $\varphi_{j i} \geq 0$. We will give some general existence, uniqueness and approximation results for nonlinear Hawkes processes, but in all the precise large-time estimates we will prove concern for the linear case.

A Hawkes process $\left(Z_{t}^{i}\right)_{i \in \mathcal{S}, t \geq 0}$ with parameters $(\mathbb{G}, \boldsymbol{\varphi}, \mathbf{h})$ behaves as follows. For each $i \in \mathcal{S}$, the rate of jump of $Z^{i}$ is, at time $t, \lambda_{i}(t)=h_{i}\left(\sum_{j \rightarrow i} \sum_{k \geq 1} \varphi_{j i}(t-\right.$ $\left.\left.T_{k}^{j}\right) \mathbf{1}_{\left\{T_{k}^{j}<t\right\}}\right)$, where $\left(T_{k}^{j}\right)_{k \geq 1}$ are the jump times of $Z^{j}$. In other words, each time one of the $Z^{j}$ 's has a jump, it excites its neighbours in that it increases their rate of jump (in the natural situation where $h$ is increasing and $\varphi$ is positive). If $\varphi$ is positive and decreases to 0 , the case of almost all applications we have in mind, the influence of a jump decreases and tends to 0 as time evolves.

1.3. Main results. In the case where $\mathbb{G}$ is a finite graph, under some appropriate assumptions on the parameters, the construction of $\left(Z_{t}^{i}\right)_{i \in \mathcal{S}, t \geq 0}$ is standard. However, for an infinite graph, the situation is more delicate: we have to check, in some sense, that the interaction does not come from infinity.

The first part of this paper (Section 2) consists of writing a Hawkes process as the solution to a system of Poisson-driven SDEs and of finding a set of assumptions on $\mathbb{G}$ and on the parameters $(\boldsymbol{\varphi}, \mathbf{h})$ under which we can prove the pathwise existence and uniqueness for this system of SDEs. Representing counting processes as solutions to SDEs is classical; see Lewis and Shedler [31], Ogata, [35], Brémaud 
and Massoulié [9], Chevallier [11]. However, the well-posedness of such SDEs is not obvious when $\mathbb{G}$ is an infinite graph.

In a second part (Section 3), we study the mean-field situation: we assume that we have a finite (large) number $N$ of particles behaving similarly, with no geometry. In other words, $\mathcal{S}=\{1, \ldots, N\}$ is endowed with the set of all possible edges $\mathcal{E}=\{(i, j): i, j \in \mathcal{S}\}$, and there are two functions $h$ and $\varphi$ such that $h_{i}=h$ and $\varphi_{i j}=N^{-1} \varphi$ for all $i, j \in \mathcal{S}$. We show that, as $N \rightarrow \infty$, Hawkes processes can be approximated by an i.i.d. family of inhomogeneous Poisson processes. Concerning the large-time behaviour, we discuss, in the linear case, the possible law of large numbers and central limit theorems as $(t, N) \rightarrow(\infty, \infty)$ and we observe some different situations according to the position of $\int_{0}^{\infty} \varphi(t) d t$ with respect to 1 (the so-called critical case).

Finally, we consider in Section 4 the case where $\mathbb{G}$ is $\mathbb{Z}^{d}$, endowed with the set of edges $\mathcal{E}=\{(i, j):|i-j|=0$ or 1$\}$, where $|\cdot|$ denotes the Euclidean distance. We study the large time behaviour, in the linear case where $h_{i}(x)=\mu_{i}+x$ and when $\varphi_{i j}=(2 d+1)^{-1} \varphi$ does not depend on $i, j$. We first assume that $\mu_{i}$ does not depend too much on $i$ (consider, e.g., the case where the $\mu_{i}$ are random, i.i.d. and bounded) and show that (i) if $\int_{0}^{\infty} \varphi(t) d t>1$, then there is a law of large numbers and the interaction makes everything flat, in the sense that for all $i \neq j, Z_{t}^{i} \sim Z_{t}^{j}$ as $t \rightarrow \infty$; (ii) if $\int_{0}^{\infty} \varphi(t) d t<1$, then there is again a law of large numbers, but the limiting value depends on $i$. We also explain why these results are reminiscent of the infinite setting and of the interaction. Finally, we study the case where $\mu_{i}=$ 0 for all $i$ but where there is an impulsion at time 0 at $i=0$. We compute the probability of extinction of such an impulsion and, in some particular cases, we study how it propagates to the whole space (when it does not blow out).

1.4. Notation. The Laplace transform of $\varphi:[0, \infty) \mapsto \mathbb{R}$ is defined, when it exists, by

$$
\mathcal{L}_{\varphi}(\alpha)=\int_{0}^{\infty} e^{-\alpha t} \varphi(t) d t .
$$

We also introduce the convolution of $h, g:[0, \infty) \mapsto \mathbb{R}$ as (if it exists) $(g \star h)_{t}=$ $\int_{0}^{t} g_{s} h_{t-s} d s=\int_{0}^{t} g_{t-s} h_{s} d s$. As is well known, when everything makes sense, $\mathcal{L}_{g \star h}(\alpha)=\mathcal{L}_{g}(\alpha) \times \mathcal{L}_{h}(\alpha)$.

2. Well-posedness using a Poisson SDE. We will study Hawkes processes through a system of Poisson-driven stochastic differential equations. This will allow us to speak of pathwise existence and uniqueness and to prove some propagation of chaos using some simple coupling arguments.

Consider, on a filtered probability space $\left(\Omega, \mathcal{F},\left(\mathcal{F}_{t}\right)_{t \geq 0}, \operatorname{Pr}\right)$, a family $\left(\pi^{i}(d s d z), i \in \mathcal{S}\right)$ of i.i.d. $\left(\mathcal{F}_{t}\right)_{t \geq 0}$-Poisson measures with intensity measure $d s d z$ on $[0, \infty) \times[0, \infty)$. 
DEFINITION 2. A family $\left(Z_{t}^{i}\right)_{i \in \mathcal{S}, t \geq 0}$ of càdlàg $\left(\mathcal{F}_{t}\right)_{t \geq 0}$-adapted processes is called a Hawkes process with parameters $(\mathbb{G}, \boldsymbol{\varphi}, \mathbf{h})$ if a.s., for all $i \in \mathcal{S}$, all $t \geq 0$

$$
Z_{t}^{i}=\int_{0}^{t} \int_{0}^{\infty} \mathbf{1}_{\left\{z \leq h_{i}\left(\sum_{j \rightarrow i} \int_{0}^{s-} \varphi_{j i}(s-u) d Z_{u}^{j}\right)\right\}} \pi^{i}(d s d z) .
$$

This formulation is consistent with Definition 1.

Proposition 3. (a) A Hawkes process in the sense of Definition 2 is also a Hawkes process in the sense of Definition 1.

(b) Consider a Hawkes process in the sense of Definition 1 (on some filtered probability space $\left.\left(\Omega, \mathcal{F},\left(\mathcal{F}_{t}\right)_{t \geq 0}, \operatorname{Pr}\right)\right)$. Then we can build, on a possibly enlarged probability space $\left(\widetilde{\Omega}, \widetilde{\mathcal{F}},\left(\widetilde{\mathcal{F}}_{t}\right)_{t \geq 0}, \widetilde{\operatorname{Pr}}\right)$, a family $\left(\pi^{i}(d s d z), i \in \mathcal{S}\right)$ of i.i.d. $\left(\widetilde{\mathcal{F}}_{t}\right)_{t \geq 0^{-}}$ Poisson measures with intensity measure $d s d z$ on $[0, \infty) \times[0, \infty)$ such that $\left(Z_{t}^{i}\right)_{i \in \mathcal{S}, t \geq 0}$ is a Hawkes process in the sense of Definition 2.

Point (a) is very easy: for a Hawkes process $\left(Z_{t}^{i}\right)_{i \in \mathcal{S}, t \geq 0}$ in the sense of Definition 2, it is clear that for every $i \in \mathcal{S}$, the compensator of $Z^{i}$ is $\int_{0}^{t} \int_{0}^{\infty} \mathbf{1}_{\left\{z \leq h_{i}\left(\sum_{j \rightarrow i} \int_{0}^{s-} \varphi_{j i}(s-u) d Z_{u}^{j}\right)\right\}} d z d s$, which is equal to $\int_{0}^{t} h_{i}\left(\sum_{j \rightarrow i} \times\right.$ $\left.\int_{0}^{s-} \varphi_{j i}(s-u) d Z_{u}^{j}\right) d s$. Furthermore, the independence of the Poisson random measures $\left(\pi^{i}(d s d z), i \in \mathcal{S}\right)$ guarantees that for all $i \neq j,\left(Z_{t}^{i}\right)_{t \geq 0}$ and $\left(Z_{t}^{j}\right)_{t \geq 0}$ a.s. never jump simultaneously.

Point (b) is more delicate but standard and a very similar result was given in Brémaud and Massoulié [9]. Their proof is based on results found in the book of Jacod [23], of which one of the main goals is exactly this topic: prove the equivalence between martingale problems and SDEs. The many results of [23] generalise those of Grigelionis [16]. See also the pioneering work of Kerstan [28]. We also refer to Chevallier [11], Section IV, where a very complete proof is given as well as a historical survey. Let us mention that the idea to integrate an indicator function with respect to a Poisson measure in order to produce an inhomogeneous Poisson process with given intensity was first introduced by Lewis and Shedler [31], and later extended by Ogata [35] in the case of a stochastic intensity.

The following set of assumptions will guarantee the well-posedness of (1).

ASSUMPTION 4. There are some nonnegative constants $\left(c_{i}\right)_{i \in \mathcal{S}}$, some positive weights $\left(p_{i}\right)_{i \in \mathcal{S}}$ and a locally integrable function $\phi:[0, \infty) \mapsto[0, \infty)$ such that:

(a) for every $i \in \mathcal{S}$, every $x, y \in \mathbb{R},\left|h_{i}(x)-h_{i}(y)\right| \leq c_{i}|x-y|$,

(b) $\sum_{i \in \mathcal{S}} h_{i}(0) p_{i}<\infty$,

(c) for every $s \in[0, \infty)$, every $j \in \mathcal{S}, \sum_{i,(j, i) \in \mathcal{E}} c_{i} p_{i}\left|\varphi_{j i}(s)\right| \leq p_{j} \phi(s)$.

Let us give a few examples of parameters $(\mathbb{G}, \boldsymbol{\varphi}, \mathbf{h})$ satisfying Assumption 4. 
REMARK 5. (i) If $\mathcal{S}$ is finite, then Assumption 4 holds true, with the choice $p_{i}=1$, as soon as $h_{i}$ is Lipschitz continuous for all $i \in \mathcal{S}$ and $\varphi_{j i}$ is locally integrable for all $(j, i) \in \mathcal{E}$.

(ii) If $\mathcal{S}=\mathbb{Z}^{d}$ is endowed with $\mathcal{E}=\{(i, j):|i-j|=0$ or 1$\}$, then Assumption 4 holds, with the choice $p_{i}=2^{-|i|}$, if $\sum_{i \in \mathbb{Z}^{d}} 2^{-|i|}\left|h_{i}(0)\right|<\infty$ and if there are $c>0$ and $\varphi \in L_{\mathrm{loc}}^{1}([0, \infty))$ such that $\left|h_{i}(x)-h_{i}(y)\right| \leq c|x-y|$ and $\left|\varphi_{j k}(t)\right| \leq \varphi(t)$ for all $i \in \mathcal{S}, x, y \in \mathbb{R},(j, k) \in \mathcal{E}$ and $t \geq 0$.

(iii) Consider next $\mathcal{S}=\mathbb{Z}^{d}$ endowed with the set of all possible edges $\mathcal{E}=$ $\left\{(i, j): i, j \in \mathbb{Z}^{d}\right\}$ and assume that there is $c>0$ such that $\left|h_{i}(0)\right| \leq c$ and $\mid h_{i}(x)-$ $h_{i}(y)|\leq c| x-y \mid$ for all $i \in \mathcal{S}, x, y \in \mathbb{R}$. Assume that there are $\varphi \in L_{\mathrm{loc}}^{1}([0, \infty))$ and a nonincreasing $a:[0, \infty) \mapsto[0, \infty)$ such that $\left|\varphi_{j i}(t)\right| \leq a(|i-j|) \varphi(t)$ for all $(i, j) \in \mathcal{E}$ and $t \geq 0$. Then if $\sum_{i \in \mathbb{Z}^{d}} a(|i|)<\infty$, Assumption 4 holds true.

(iv) Consider the (strongly oriented) graph $\mathbb{Z}_{+}$endowed with the set of edges $\mathcal{E}=\left\{(i, i+1): i \in \mathbb{Z}_{+}\right\}$. Then Assumption 4 holds true as soon as there is $\varphi \in$ $L_{\text {loc }}^{1}([0, \infty))$ such that for every $i \in \mathbb{Z}_{+}$, there are $c_{i}>0$ and $a_{i}>0$ such that $\left|h_{i}(x)-h_{i}(y)\right| \leq c_{i}|x-y|$ and $\left|\varphi_{i(i+1)}\right| \leq a_{i} \varphi$.

Points (ii) and (iii) of course extend to other graphs. In (iv), there is no growth condition on $\left|h_{i}(0)\right|, c_{i}$ and $a_{i}$. This comes from the fact that the interaction is directed: $Z^{0}$ is actually a Poisson process with rate $h_{0}(0)$, the intensity of $Z^{1}$ is entirely determined by that of $Z^{0}$, and so on. Hence, this example is not very interesting. But we can mix (ii) and (iv): coefficients connected to the edges that are directed to the origin have to be controlled.

ProOF OF REMARK 5. Point (i) is obvious. To check (ii), simply note that for all $j \in \mathcal{S}, \sum_{i,(j, i) \in \mathcal{E}} c 2^{-|i|}\left|\varphi_{j i}\right| \leq c \varphi 2^{-|j|} \sum_{i,(j, i) \in \mathcal{E}} 2^{|j|-|i|} \leq c 2(2 d+1) \varphi 2^{-|j|}$ and define $\phi=c 2(2 d+1) \varphi$. Point (iv) holds with $\left(p_{i}\right)_{i \in \mathbb{Z}_{+}}$defined by $p_{0}=1$ and, by induction, $p_{i+1}=\min \left\{2^{-i} /\left(1+h_{i+1}(0)\right), p_{i} /\left(1+a_{i} c_{i+1}\right)\right\}$. This of course implies that $\sum_{i \in \mathbb{Z}_{+}} p_{i}\left|h_{i}(0)\right|<\infty$ and that for all $j \geq 1, \sum_{i,(j, i) \in \mathcal{E}} c_{i} p_{i}\left|\varphi_{j i}\right|=$ $c_{j+1} p_{j+1}\left|\varphi_{j(j+1)}\right| \leq c_{j+1} p_{j+1} a_{j} \varphi \leq p_{j} \varphi$ as desired.

To prove (iii), we work with the sup norm $|i|=\left|\left(i_{1}, \ldots, i_{d}\right)\right|=\max \left\{\left|i_{1}\right|, \ldots\right.$, $\left.\left|i_{d}\right|\right\}$. The delicate part consists in showing that there is $b: \mathbb{N} \mapsto[0, \infty)$ and a constant $C>0$ such that $\sum_{i \in \mathbb{Z}^{d}} b(|i|)<\infty$ and, for all $j \in \mathbb{Z}^{d}, \sum_{i \in \mathbb{Z}^{d}} b(|i|) a(\mid i-$ $j \mid) \leq C b(|j|)$. Then the result will easily follow, with the choices $p_{i}=b(|i|)$ and $\phi=C c \varphi$. We define $b$ recursively, by $b(0)=a(0)$ and $b(k+1)=\max \{a(k+$ $\left.1),[(k+1) /(k+2)]^{2 d} b(k)\right\}$. Using that $a$ is nonincreasing, we easily check that $b$ is nonincreasing. We next check that $\sum_{i \in \mathbb{Z}^{d}} b(|i|)<\infty$, that is, $\sum_{k \geq 0} k^{d-1} b(k)<$ $\infty$, knowing by assumption that $\sum_{k \geq 0} k^{d-1} a(k)<\infty$. We have, for $k \geq 0$,

$$
\begin{aligned}
b(k+1)-a(k+1) & \\
& =\left([(k+1) /(k+2)]^{2 d} b(k)-a(k+1)\right)_{+} \\
& \leq[(k+1) /(k+2)]^{2 d}(b(k)-a(k))_{+}
\end{aligned}
$$




$$
\begin{aligned}
& +\left([(k+1) /(k+2)]^{2 d} a(k)-a(k+1)\right)_{+} \\
\leq & {[(k+1) /(k+2)]^{2 d}(b(k)-a(k))+(a(k)-a(k+1)) . }
\end{aligned}
$$

Recalling that $b(0)=a(0)$, one gets $b(k)-a(k) \leq \sum_{\ell=1}^{k}(a(\ell-1)-a(\ell))[(\ell+$ $1) /(k+1)]^{2 d}$ by iteration. Hence,

$$
\begin{aligned}
\sum_{k \geq 1} k^{d-1}(b(k)-a(k)) & \leq \sum_{k \geq 1} k^{d-1} \sum_{\ell=1}^{k}(a(\ell-1)-a(\ell))[(\ell+1) /(k+1)]^{2 d} \\
& =\sum_{\ell \geq 1}(a(\ell-1)-a(\ell))(\ell+1)^{2 d} \sum_{k \geq \ell} k^{d-1}(k+1)^{-2 d} \\
& \leq C \sum_{\ell \geq 1}(a(\ell-1)-a(\ell)) \ell^{d} .
\end{aligned}
$$

This last quantity is nothing but $C \sum_{\ell \geq 1} a(\ell)\left[(\ell+1)^{d}-\ell^{d}\right] \leq C \sum_{\ell \geq 1} a(\ell) \ell^{d-1}<$ $\infty$. We have thus checked that $\sum_{k \geq 0} k^{d-1} b(k)=\sum_{k \geq 1} a(k) k^{d-1}+\sum_{k \geq 1} k^{d-1} \times$ $(b(k)-a(k))<\infty$.

We finally prove that for all $j \in \mathbb{Z}^{d}, \sum_{i \in \mathbb{Z}^{d}} b(|i|) a(|i-j|) \leq C b(|j|)$. First, we claim that there is $C$ such that $b(k) \leq C b(2 k)$ for all $k \geq 0$. This is easily checked, iterating the inequality $b(k) \leq[(k+2) /(k+1)]^{2 d} b(k+1)$. Next we write, using that $a$ and $b$ are nonincreasing,

$$
\begin{aligned}
\sum_{i \in \mathbb{Z}^{d}} b(|i|) a(|i-j|) & \leq \sum_{|i|<|j| / 2} b(|i|) a(|i-j|)+\sum_{|i| \geq|j| / 2} b(|i|) a(|i-j|) \\
& \leq a(|j| / 2) \sum_{i \in \mathbb{Z}^{d}} b(|i|)+b(|j| / 2) \sum_{i \in \mathbb{Z}^{d}} a(|i-j|) \\
& \leq C a(|j| / 2)+C b(|j| / 2) .
\end{aligned}
$$

By definition of $b$, we have $a(|j| / 2) \leq b(|j| / 2)$. And we have just seen that $b(|j| / 2) \leq C b(|j|)$. We finally have checked that $\sum_{i \in \mathbb{Z}^{d}} b(|i|) a(|i-j|) \leq C b(|j|)$ as desired.

Our well-posedness result is the following.

THEOREM 6. Under Assumption 4, there exists a pathwise unique Hawkes process $\left(Z_{t}^{i}\right)_{i \in \mathcal{S}, t \geq 0}$ such that $\sum_{i \in \mathcal{S}} p_{i} \mathbb{E}\left[Z_{t}^{i}\right]<\infty$ for all $t \geq 0$.

Observe that this result is not completely obvious in the case of an infinite graph. In some sense, we have to check that the interaction does not come from infinity. Let us insist on the fact that, even in simple situations, a graphical construction is 
not possible: consider, for example, the case of $\mathbb{Z}$ endowed with the set of edges $\mathcal{E}=\{(i, j):|i-j|=0$ or 1$\}$, assume that $h_{i}(x)=1+x$ for all $i \in \mathcal{S}$ and that $\varphi_{i j}=1$ for all $(i, j) \in \mathcal{E}$. Then one easily gets convinced that we cannot determine the values of $\left(Z_{t}^{0}\right)_{t \in[0, T]}$ by observing the Poisson measures $\pi^{i}$ in a (random) finite box.

As a second comment, let us mention that we believe it is not possible, or at least quite difficult, to obtain the full uniqueness, that is, uniqueness outside the class of processes satisfying $\sum_{i \in \mathcal{S}} p_{i} \mathbb{E}\left[Z_{t}^{i}\right]<\infty$ (or something similar). Indeed, consider again the case of $\mathbb{Z}$ endowed with $\mathcal{E}=\{(i, j):|i-j|=0$ or 1$\}$, assume that $h_{i}(x)=1+x$ for all $i \in \mathcal{S}$ and that $\varphi_{j i}=1$ for all $(i, j) \in \mathcal{E}$. One easily checks that for $\left(Z_{t}^{i}\right)_{i \in \mathcal{S}, t \geq 0}$ a Hawkes process, for $m_{t}^{i}=\mathbb{E}\left[Z_{t}^{i}\right]$, it holds that $m_{t}^{i}=t+$ $\int_{0}^{t}\left(m_{s}^{i-1}+m_{s}^{i}+m_{s}^{i+1}\right) d s$ for every $i$. This infinite system of equations is of course closely related to the heat equation $\partial_{t} u(t, x)=1+\partial_{x x} u(t, x)$ on $[0, \infty) \times \mathbb{R}$ and with initial condition $u(0, x)=0$. As is well known, uniqueness for this equation fails to hold true without imposing some growth conditions as $|x| \rightarrow \infty$. See, for example, Tychonov's counterexample of uniqueness, which can be found in John [26], Chapter 7.

To compare Theorem 6 with the results of Massoulié [32], let us consider a very simple situation. Let $\mathcal{S}=\mathbb{Z}^{d}$ be endowed with $\mathcal{E}=\{(i, j):|i-j|=0$ or $1\}$, let $h_{i}(x)=1+x$ for all $i \in \mathcal{S}$ and $\varphi_{i j}(t)=\varphi(t)>0$ for all $(i, j) \in \mathcal{E}$. Then Theorem 6 applies as soon as $\varphi \in L_{\text {loc }}^{1}([0, \infty))$; see Remark 5(ii). Theorem 1 of [32] does not apply for two reasons: $\sup _{i \in \mathcal{S}} h_{i}$ is not bounded and, more important, it does not hold true that $\sum_{(i, j) \in \mathcal{E}} \varphi_{i j} \in L_{\text {loc }}^{1}([0, \infty))$ since $\sum_{(i, j) \in \mathcal{E}} \varphi_{i j}(t)=+\infty$ for all $t \geq 0$. On the contrary, [32], Theorem 2, applies, but only in the subcritical case where $(2 d+1) \int_{0}^{\infty} \varphi(t) d t<1$.

Proof of Theorem 6. We first prove uniqueness. Let thus $\left(Z_{t}^{i}\right)_{i \in \mathcal{S}, t \geq 0}$ and $\left(\widetilde{Z}_{t}^{i}\right)_{i \in \mathcal{S}, t \geq 0}$ be two solutions to (1) satisfying the required condition. Set

$$
\Delta_{t}^{i}=\int_{0}^{t}\left|d\left(Z_{s}^{i}-\widetilde{Z}_{s}^{i}\right)\right| \quad \text { for } i \in \mathcal{S}, t \geq 0 .
$$

In other words, $\Delta_{t}^{i}$ is the total variation norm of the signed measure $d\left(Z_{s}^{i}-\widetilde{Z}_{s}^{i}\right)$ on $[0, t]$. We also put $\delta_{t}^{i}=\mathbb{E}\left[\Delta_{t}^{i}\right]$ and first prove that

$$
\delta_{t}^{i} \leq c_{i} \int_{0}^{t} \sum_{j \rightarrow i}\left|\varphi_{j i}(t-s)\right| \delta_{s}^{j} d s .
$$

We have

$$
\begin{aligned}
\Delta_{t}^{i}=\int_{0}^{t} \int_{0}^{\infty} \mid & \mathbf{1}_{\left\{z \leq h_{i}\left(\sum_{j \rightarrow i} \int_{0}^{s-} \varphi_{j i}(s-u) d Z_{u}^{j}\right)\right\}} \\
& -\mathbf{1}_{\left\{z \leq h_{i}\left(\sum_{j \rightarrow i} \int_{0}^{s-} \varphi_{j i}(s-u) d \widetilde{Z}_{u}^{j}\right)\right\}} \mid \pi^{i}(d s d z) .
\end{aligned}
$$


Taking expectations, we deduce that

$$
\begin{aligned}
\delta_{t}^{i}=\int_{0}^{t} \mathbb{E}\left[\mid h_{i}\left(\sum_{j \rightarrow i} \int_{0}^{s-} \varphi_{j i}(s-u) d Z_{u}^{j}\right)\right. \\
\left.-h_{i}\left(\sum_{j \rightarrow i} \int_{0}^{s-} \varphi_{j i}(s-u) d \widetilde{Z}_{u}^{j}\right) \mid\right] d s \\
\leq c_{i} \sum_{j \rightarrow i} \mathbb{E}\left[\int_{0}^{t} \int_{0}^{s-}\left|\varphi_{j i}(s-u)\right| d \Delta_{u}^{j} d s\right]
\end{aligned}
$$

by Assumption 4(a). Using Lemma 22, we see that

$$
\int_{0}^{t} d s \int_{0}^{s-}\left|\varphi_{j i}(s-u)\right| d \Delta_{u}^{j}=\int_{0}^{t}\left|\varphi_{j i}(t-u)\right| \Delta_{u}^{j} d u
$$

which, plugged into (3), yields (2).

Set $\delta_{t}=\sum_{i \in \mathcal{S}} p_{i} \delta_{t}^{i}$, where the weights $p_{i}$ were introduced in Assumption 4. By assumption, $\delta_{t}$ is well defined and finite. We infer by (2) that

$$
\delta_{t} \leq \int_{0}^{t} \sum_{i \in \mathcal{S}} p_{i} c_{i} \sum_{j \rightarrow i}\left|\varphi_{j i}(t-s)\right| \delta_{s}^{j} d s
$$

By Assumption 4(c),

$$
\begin{aligned}
\delta_{t} & \leq \int_{0}^{t} \sum_{j \in \mathcal{S}} \delta_{s}^{j} \sum_{i,(j, i) \in \mathcal{E}} c_{i} p_{i}\left|\varphi_{j i}(t-s)\right| d s \leq \int_{0}^{t} \sum_{j \in \mathcal{S}} p_{j} \delta_{s}^{j} \phi(t-s) d s \\
& =\int_{0}^{t} \phi(t-s) \delta_{s} d s .
\end{aligned}
$$

Lemma 23(i) thus implies that $\delta_{t}=0$ identically, from which uniqueness follows.

We now quickly prove existence by a Picard iteration. Let $Z_{t}^{i, 0}=0$ and, for $n \geq 0$,

$$
Z_{t}^{i, n+1}=\int_{0}^{t} \int_{0}^{\infty} \mathbf{1}_{\left\{z \leq h_{i}\left(\sum_{j \rightarrow i} \int_{0}^{s-} \varphi_{j i}(s-u) d Z_{u}^{j, n}\right)\right\}} \pi^{i}(d s d z) .
$$

We define $\delta_{t}^{i, n}=\mathbb{E}\left[\int_{0}^{t}\left|d Z_{s}^{i, n+1}-d Z_{s}^{i, n}\right|\right]$ and $\delta_{t}^{n}=\sum_{i \in \mathcal{S}} p_{i} \delta_{t}^{i, n}$. As in the proof of uniqueness, we obtain, for $n \geq 0$,

$$
\delta_{t}^{n+1} \leq \int_{0}^{t} \phi(t-s) \delta_{s}^{n} d s .
$$

Next, we put $m_{t}^{i, n}=\mathbb{E}\left[Z_{t}^{i, n}\right]$. By Assumption $4(\mathrm{a}), h_{i}(x) \leq h_{i}(0)+c_{i}|x|$, whence

$$
\begin{aligned}
m_{t}^{i, n+1} & \leq \mathbb{E}\left[\int_{0}^{t}\left(h_{i}(0)+c_{i} \sum_{j \rightarrow i} \int_{0}^{s-}\left|\varphi_{j i}(s-u)\right| d Z_{u}^{j, n}\right) d s\right] \\
& \leq \int_{0}^{t}\left(h_{i}(0)+c_{i} \sum_{j \rightarrow i}\left|\varphi_{j i}(t-s)\right| m_{s}^{j, n}\right) d s,
\end{aligned}
$$


where we used that, by Lemma $22, \int_{0}^{t} \int_{0}^{s-}\left|\varphi_{j i}(s-u)\right| d Z_{u}^{j, n} d s=\int_{0}^{t} \mid \varphi_{j i}(t-$ $u) \mid Z_{u}^{j, n} d u$. Setting $u_{t}^{n}=\sum_{i \in \mathcal{S}} p_{i} m_{t}^{i, n}$ and using Assumption 4(b)-(c),

$$
\begin{aligned}
u_{t}^{n+1} & \leq t \sum_{i \in \mathcal{S}} h_{i}(0) p_{i}+\int_{0}^{t} \sum_{i \in \mathcal{S}} p_{i} c_{i} \sum_{j \rightarrow i}\left|\varphi_{j i}(s-u)\right| m_{s}^{j, n} d s \\
& \leq C t+\int_{0}^{t} \phi(t-s) u_{s}^{n} d s .
\end{aligned}
$$

Since $u_{t}^{0}=0$ and $\phi$ is locally integrable, we easily check by induction that $u^{n}$ is locally bounded for all $n \geq 0$. Consequently, $\delta^{n}$ is also locally bounded for all $n \geq 0$. Lemma 23(ii) implies that for all $T \geq 0, \sum_{n \geq 1} \delta_{T}^{n}<\infty$. This classically implies that the Picard sequence is Cauchy, and thus converges: there exists a family $\left(Z_{t}^{i}\right)_{i \in \mathcal{S}, t \geq 0}$ of càdlàg nonnegative adapted processes such that for all $T \geq 0, \lim _{n} \sum_{i \in \mathcal{S}} p_{i} \mathbb{E}\left[\int_{0}^{T}\left|d Z_{s}^{i}-d Z_{s}^{i, n}\right|\right]=0$. It is then not hard to pass to the limit in (4) to deduce that $\left(Z_{t}^{i}\right)_{i \in \mathcal{S}, t \geq 0}$ solves (1). Finally, Lemma 23(iii) implies that $\sup _{n} u_{t}^{n}<\infty$ for all $t \geq 0$, from which $\sum_{i \in \mathcal{S}} p_{i} \mathbb{E}\left[Z_{t}^{i}\right]<\infty$ as desired.

3. Mean-field limit. In this section, we work in the following setting.

Assumption 7. Let $h: \mathbb{R} \mapsto[0, \infty)$ be such that $|h|_{\text {lip }}=\sup _{x \neq y} \mid x-$ $\left.y\right|^{-1}|h(x)-h(y)|<\infty$ and let $\varphi=[0, \infty) \mapsto \mathbb{R}$ be a locally square integrable function.

For each $N \geq 1$, we consider the complete graph $\mathbb{G}_{N}$ with vertices $\mathcal{S}_{N}=$ $\{1, \ldots, N\}$ and edges $\mathcal{E}_{N}=\left\{(i, j): i, j \in \mathcal{S}_{N}\right\}$, that is, all pairs of points in $\mathcal{S}_{N}$ are connected. We put $h_{i}^{N}=h$ for all $i \in \mathcal{S}_{N}$ and $\varphi_{j i}^{N}=N^{-1} \varphi$ for $(i, j) \in \mathcal{E}_{N}$.

Under Assumption 7, the triplet $\left(\mathbb{G}_{N}, \boldsymbol{\varphi}^{N}, \mathbf{h}^{N}\right)$ satisfies Assumption 4 (the graph $\mathbb{G}_{N}$ is finite) for each $N \geq 1$. Therefore, a Hawkes process $\left(Z_{t}^{N, 1}, \ldots\right.$, $\left.Z_{t}^{N, N}\right)_{t \geq 0}$ with parameters $\left(\mathbb{G}_{N}, \varphi^{N}, \mathbf{h}^{N}\right)$ is uniquely defined by Theorem 6.

Introduce the limit equation

$$
\bar{Z}_{t}=\int_{0}^{t} \int_{0}^{\infty} \mathbf{1}_{\left\{z \leq h\left(\int_{0}^{s} \varphi(s-u) d \mathbb{E}\left[\bar{Z}_{u}\right]\right)\right\}} \pi(d s d z) \quad \text { for every } t \geq 0,
$$

where $\pi(d s d z)$ is a Poisson measure on $[0, \infty) \times[0, \infty)$ with intensity measure $d s d z$. Also, $d \mathbb{E}\left[\bar{Z}_{u}\right]$ is the measure on $[0, \infty)$ associated to the (necessarily) nondecreasing function $u \mapsto \mathbb{E}\left[\bar{Z}_{u}\right]$. Note that a solution $\bar{Z}=\left(\bar{Z}_{t}\right)_{t \geq 0}$, if it exists, is an inhomogeneous Poisson process on $[0, \infty)$ with intensity $\lambda_{t}=$ $h\left(\int_{0}^{t} \varphi(t-u) d \mathbb{E}\left[\bar{Z}_{u}\right]\right)$.

3.1. Propagation of chaos. The main result of this section reads as follows. We denote by $\mathbb{D}([0, \infty), \mathbb{R})$ the set of càdlàg $\mathbb{R}$-valued functions on $[0, \infty)$ and by $\mathcal{P}(\mathbb{D}([0, \infty), \mathbb{R}))$ the set of probability measures on $\mathbb{D}([0, \infty), \mathbb{R})$. 
THEOREM 8. Work under Assumption 7.

(i) There is a pathwise unique solution $\left(\bar{Z}_{t}\right)_{t \geq 0}$ to $(7)$ such that $\left(\mathbb{E}\left[\bar{Z}_{t}\right]\right)_{t \geq 0}$ is locally bounded.

(ii) It is possible to build simultaneously the Hawkes process $\left(Z_{t}^{N, 1}, \ldots\right.$, $\left.Z_{t}^{N, N}\right)_{t \geq 0}$ with parameters $\left(\mathbb{G}_{N}, \varphi^{N}, \mathbf{h}^{N}\right)$ and an i.i.d. family $\left(\bar{Z}_{t}^{i}\right)_{t \geq 0, i=1, \ldots, N}$ of solutions to (7) in such a way that for all $T>0$, all $i=1, \ldots, N$,

$$
\mathbb{E}\left[\sup _{[0, T]}\left|Z_{t}^{N, i}-\bar{Z}_{t}^{i}\right|\right] \leq C_{T} N^{-1 / 2}
$$

the constant $C_{T}$ depending only on $h, \varphi$ and $T$ (see Remark 9 below for some bounds of $C_{T}$ in a few situations).

(iii) Consequently, we have the mean-field approximation

$$
\frac{1}{N} \sum_{i=1}^{N} \delta_{\left(Z_{t}^{N, i}\right)_{t \geq 0}} \longrightarrow \mathcal{L}\left(\left(\bar{Z}_{t}\right)_{t \geq 0}\right) \quad \text { in probability, as } N \rightarrow \infty,
$$

where $\mathcal{P}(\mathbb{D}([0, \infty), \mathbb{R}))$ is endowed with the weak convergence topology associated with the topology $($ on $\mathbb{D}([0, \infty), \mathbb{R}))$ of the uniform convergence on compact time intervals.

PROOF. For $\left(\bar{Z}_{t}\right)_{t \geq 0}$ a solution to (7), the equation satisfied by $m_{t}=\mathbb{E}\left[\bar{Z}_{t}\right]$ writes

$$
m_{t}=\int_{0}^{t} h\left(\int_{0}^{s} \varphi(s-u) d m_{u}\right) d s \quad \text { for every } t \geq 0 .
$$

By Lemma 24, we know that this equation has a unique nondecreasing locally bounded solution, which furthermore is of class $C^{1}$ on $[0, \infty)$. We now split the proof in several steps.

Step 1. Here we prove the well-posedness of (7). For $\left(\bar{Z}_{t}\right)_{t \geq 0}$ a solution to (7), its expectation $m_{t}=\mathbb{E}\left[\bar{Z}_{t}\right]$ solves (8) and is thus uniquely defined. Thus, the right-hand side of (7) is uniquely determined, which proves uniqueness. For the existence, consider $m$ the unique solution to (8) and put $\bar{Z}_{t}=$ $\int_{0}^{t} \int_{0}^{\infty} \mathbf{1}_{\left\{z \leq h\left(\int_{0}^{s} \varphi(s-u) d m_{u}\right)\right\}} \pi(d s d z)$. We thus only have to prove that $\mathbb{E}\left[\bar{Z}_{t}\right]=m_{t}$. But $\mathbb{E}\left[\bar{Z}_{t}\right]=\int_{0}^{t} h\left(\int_{0}^{s} \varphi(s-u) d m_{u}\right) d s$, which is nothing but $m_{t}$ since $m$ solves (8).

Step 2. We next introduce a suitable coupling. Let $\left(\pi^{i}(d s d z)\right)_{i \geq 1}$ be an i.i.d. family of Poisson measures with common intensity measure $d s d z$ on $[0, \infty) \times$ $[0, \infty)$. For each $N \geq 1$, we consider the Hawkes process $\left(Z_{t}^{N, 1}, \ldots, Z_{t}^{N, N}\right)_{t \geq 0}$

$$
Z_{t}^{N, i}=\int_{0}^{t} \int_{0}^{\infty} \mathbf{1}_{\left\{z \leq h\left(N^{-1} \sum_{j=1}^{N} \int_{0}^{s-} \varphi(s-u) d Z_{u}^{N, j}\right)\right\}} \pi^{i}(d s d z) .
$$

Next, still denoting by $m$ the unique solution to (8), we put, for every $i \geq 1$,

$$
\bar{Z}_{t}^{i}=\int_{0}^{t} \int_{0}^{\infty} \mathbf{1}_{\left\{z \leq h\left(\int_{0}^{s-} \varphi(s-u) d m_{u}\right)\right\}} \pi^{i}(d s d z) .
$$

Clearly, $\left(\left(\bar{Z}_{t}^{i}\right)_{t \geq 0}\right)_{i \geq 1}$ is an i.i.d. family of solutions to (7). 
Step 3. Here we introduce $\Delta_{N}^{i}(t)=\int_{0}^{t}\left|d\left(\bar{Z}_{u}^{i}-Z_{u}^{N, i}\right)\right|$ and $\delta_{N}(t)=\mathbb{E}\left[\Delta_{N}^{i}(t)\right]$, which obviously does not depend on $i$ (by exchangeability). Observe that

(9) $\sup _{[0, t]}\left|\bar{Z}_{u}^{i}-Z_{u}^{N, i}\right| \leq \Delta_{N}^{i}(t) \quad$ whence $\mathbb{E}\left[\sup _{[0, t]}\left|\bar{Z}_{u}^{i}-Z_{u}^{N, i}\right|\right] \leq \delta_{N}(t)$.

The first inequality follows from the fact that $\left|\bar{Z}_{u}^{i}-Z_{u}^{N, i}\right| \leq\left|\int_{0}^{u} d\left(\bar{Z}_{r}^{i}-Z_{r}^{N, i}\right)\right| \leq$ $\int_{0}^{u}\left|d\left(\bar{Z}_{r}^{i}-Z_{r}^{N, i}\right)\right|$. We show in this step that for all $t>0$,

$$
\begin{aligned}
\delta_{N}(t) \leq & |h|_{\operatorname{lip}} N^{-1 / 2} \int_{0}^{t}\left(\int_{0}^{s} \varphi^{2}(s-u) d m_{u}\right)^{1 / 2} d s \\
& +|h|_{\text {lip }} \int_{0}^{t}|\varphi(t-s)| \delta_{N}(s) d s .
\end{aligned}
$$

First, $\Delta_{N}^{1}(t)$ equals

$$
\int_{0}^{t} \int_{0}^{\infty}\left|\mathbf{1}_{\left\{z \leq h\left(N^{-1} \sum_{j=1}^{N} \int_{0}^{s-} \varphi(s-u) d Z_{u}^{N, j}\right)\right\}}-\mathbf{1}_{\left\{z \leq h\left(\int_{0}^{s-} \varphi(s-u) d m_{u}\right)\right\}}\right| \pi^{i}(d s d z) .
$$

Taking expectations, we find

$$
\delta_{N}(t)=\int_{0}^{t} \mathbb{E}\left[\left|h\left(\int_{0}^{s} \varphi(s-u) d m_{u}\right)-h\left(N^{-1} \sum_{j=1}^{N} \int_{0}^{s} \varphi(s-u) d Z_{u}^{N, j}\right)\right|\right] d s,
$$

whence

$$
\begin{aligned}
\delta_{N}(t) \leq & |h|_{\text {lip }} \int_{0}^{t} \mathbb{E}\left[\left|\int_{0}^{s} \varphi(s-u) d m_{u}-N^{-1} \sum_{j=1}^{N} \int_{0}^{s} \varphi(s-u) d \bar{Z}_{u}^{j}\right|\right] d s \\
& +|h|_{\text {lip }} \int_{0}^{t} \mathbb{E}\left[\left|N^{-1} \sum_{j=1}^{N} \int_{0}^{s} \varphi(s-u) d\left[\bar{Z}_{u}^{j}-Z_{u}^{N, j}\right]\right|\right] d s \\
= & |h|_{\text {lip }}(A+B) .
\end{aligned}
$$

Using exchangeability and Lemma 22,

$$
B \leq \int_{0}^{t} \mathbb{E}\left[\int_{0}^{s}|\varphi(s-u)| d \Delta_{N}^{1}(u)\right] d s=\int_{0}^{t}|\varphi(t-u)| \delta_{N}(u) d u .
$$

Next, we use that $X_{s}^{j}=\int_{0}^{s} \varphi(s-u) d \bar{Z}_{u}^{j}$ are i.i.d. with mean $\int_{0}^{s} \varphi(s-u) d m_{u}$, whence

$$
A \leq N^{-1 / 2} \int_{0}^{t}\left(\operatorname{Var} X_{s}^{1}\right)^{1 / 2} d s
$$

But it holds that

$$
X_{s}^{1}=\int_{0}^{s} \int_{0}^{\infty} \mathbf{1}_{\left\{z \leq h\left(\int_{0}^{u} \varphi(u-r) d m_{r}\right)\right\}} \varphi(s-u) \pi^{1}(d u d z) .
$$


Since the integrand is deterministic, denoting by $\tilde{\pi}^{1}$ the compensated Poisson measure,

$$
X_{s}^{1}-\mathbb{E}\left[X_{s}^{1}\right]=\int_{0}^{s} \int_{0}^{\infty} \mathbf{1}_{\left\{z \leq h\left(\int_{0}^{u} \varphi(u-r) d m_{r}\right)\right\}} \varphi(s-u) \tilde{\pi}^{1}(d u d z) .
$$

Recalling Assumption 7, we find

$$
\operatorname{Var} X_{s}^{1}=\int_{0}^{s} \varphi^{2}(s-u) h\left(\int_{0}^{u} \varphi(u-r) d m_{r}\right) d u=\int_{0}^{s} \varphi^{2}(s-u) d m_{u} .
$$

We used (8) for the last equality. Gathering (11), (12), (13) and (14) completes the step.

Step 4. Here, we conclude that for all $T \geq 0, \sup _{[0, T]} \delta_{N}(t) \leq C_{T} N^{-1 / 2}$. This will complete the proof of (ii) by (9). This is not hard: it suffices to start from (10), to apply Lemma 23(i) and to observe that $\int_{0}^{t}\left(\int_{0}^{s} \varphi^{2}(s-u) d m_{u}\right)^{1 / 2} d s$ is locally bounded (which follows from the assumption that $\varphi$ is locally square integrable and the fact that $m$ is $C^{1}$ on $[0, \infty)$ ).

Step 5. Finally, (iii) follows from (ii): by Sznitman [43], Proposition 2.2, it suffices to check that for each fixed $\ell \geq 1,\left(\left(Z_{t}^{N, 1}\right)_{t \geq 0}, \ldots,\left(Z_{t}^{N, \ell}\right)_{t \geq 0}\right)$ goes in law, as $N \rightarrow \infty$, to $\ell$ independent copies of $\left(\bar{Z}_{t}\right)_{t \geq 0}$ (for the uniform topology on compact time intervals). This clearly follows from (ii).

We now want to show that the constant $C_{T}$ we get can be quite satisfactory.

REMARK 9. Work under Assumption 7.

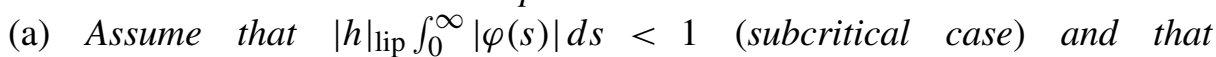
$\int_{0}^{\infty} \varphi^{2}(s) d s<\infty$. Then (ii) of Theorem 8 holds with $C_{T}=C T$, for some constant $C>0$. This is a satisfactory slow growth.

(b) Assume that $h(x)=\mu+x$ for some $\mu>0$ and that $\varphi(t)=a e^{-b t}$ for some $a>b>0$ [if $a<b$, then point (a) applies]. Then $m_{t}=\mathbb{E}\left[\bar{Z}_{t}\right] \sim \mu a(a-$ $b)^{-2} e^{(a-b) t}$ as $t \rightarrow \infty$ and (ii) of Theorem 8 holds with $C_{T}=C e^{(a-b) T}$, for some constant $C>0$. This is again quite satisfactory: the error is of order $N^{-1 / 2} m_{T}$.

PROOF. We start with (a). Using the notation of the previous proof, it suffices [see (9)] to show that $\delta_{N}(T) \leq C T N^{-1 / 2}$. Setting $\Lambda=|h|_{\text {lip }} \int_{0}^{\infty}|\varphi(s)| d s<1$, starting from (10) and observing that $\delta_{N}$ is nondecreasing, we find $\delta_{N}(t) \leq$ $|h|_{\operatorname{lip}} N^{-1 / 2} \int_{0}^{t}\left(\int_{0}^{s} \varphi^{2}(s-u) d m_{u}\right)^{1 / 2} d s+\Lambda \delta_{N}(t)$, whence $\delta_{N}(t) \leq C N^{-1 / 2} \times$ $\int_{0}^{t}\left(\int_{0}^{s} \varphi^{2}(s-u) d m_{u}\right)^{1 / 2} d s$. We thus only have to check that $\int_{0}^{s} \varphi^{2}(s-u) d m_{u}$ is bounded on $[0, \infty)$. Since $\int_{0}^{\infty} \varphi^{2}(s) d s<\infty$, it suffices to prove that $m^{\prime}$ is bounded on $[0, \infty)$. But $m_{t}^{\prime}=h\left(\int_{0}^{t} \varphi(t-u) m_{u}^{\prime} d u\right) \leq h(0)+|h|_{\operatorname{lip}} \int_{0}^{t}|\varphi(t-u)| m_{u}^{\prime} d u$, whence $\sup _{[0, T]} m_{t}^{\prime} \leq h(0)+\Lambda \sup _{[0, T]} m_{t}^{\prime}$, and thus $\sup _{[0, T]} m_{t}^{\prime} \leq h(0) /(1-\Lambda)$ for any $T>0$. 
We next check (b). First, (8) rewrites $m_{t}=\mu t+a \int_{0}^{t} \int_{0}^{s} e^{-b(s-u)} d m_{u} d s$, with unique solution

$$
m_{t}=\frac{-\mu b t}{a-b}+\frac{\mu a\left(e^{(a-b) t}-1\right)}{(a-b)^{2}} \sim \frac{\mu a}{(a-b)^{2}} e^{(a-b) t} .
$$

Next, using (10) and the explicit expressions of $h, \varphi$ and $m$, we find

$$
\begin{aligned}
\delta_{N}(t) & \leq N^{-1 / 2} \int_{0}^{t}\left(\int_{0}^{s} \varphi^{2}(s-u) d m_{u}\right)^{1 / 2} d s+\int_{0}^{t} \varphi(t-s) \delta_{N}(s) d s \\
& \leq C N^{-1 / 2} e^{(a-b) t / 2}+a \int_{0}^{t} e^{-b(t-s)} \delta_{N}(s) d s .
\end{aligned}
$$

Setting $u_{N}(t)=\delta_{N}(t) e^{b t}$, we get $u_{N}(t) \leq C N^{-1 / 2} e^{(a+b) t / 2}+a \int_{0}^{t} u_{N}(s) d s$. By Grönwall's lemma, $u_{N}(t) \leq C N^{-1 / 2} e^{(a+b) t / 2}+a \int_{0}^{t} C N^{-1 / 2} e^{(a+b) s / 2} e^{a(t-s)} d s$. On easily deduces, since $a>b$, that $u_{N}(t) \leq C N^{-1 / 2} e^{a t}$ so that $\delta_{N}(t) \leq$ $C N^{-1 / 2} e^{(a-b) t}$. The use of (9) completes the proof.

3.2. Large time behaviour. We now address the important problem of the large time behaviour. Since the solution $\left(\bar{Z}_{t}\right)_{t \geq 0}$ to $(7)$ is nothing but an inhomogeneous Poisson process, its large-time behaviour is easily and precisely described, provided we have sufficiently information on the solution to (8). The question is thus: can we use the large time estimates of the mean-field limit to describe the largetime behaviour of the true Hawkes process with a large number of particles? To fix the ideas, we consider the linear case. For $A$ a symmetric nonnegative matrix, we denote by $\mathcal{N}(0, A)$ the centered Gaussian distribution with covariance matrix $A$.

We treat separately the subcritical and supercritical cases.

THEOREM 10. Work under Assumption 7 with $\varphi$ nonnegative and $h(x)=\mu+$ $x$ for some $\mu>0$. Assume also that $\Lambda=\int_{0}^{\infty} \varphi(s) d s<1$. For each $N \geq 1$, consider the Hawkes process $\left(Z_{t}^{N, 1}, \ldots, Z_{t}^{N, N}\right)_{t \geq 0}$ with parameters $\left(\mathbb{G}_{N}, \varphi^{N}, \mathbf{h}^{N}\right)$. Consider also the unique solution $\left(m_{t}\right)_{t \geq 0}$ to $(8)$ :

1. We have $m_{t} \sim a_{0} t$ as $t \rightarrow \infty$, where $a_{0}=\mu /(1-\Lambda)$.

2. For any fixed $i \geq 1, Z_{t}^{N, i} / m_{t}$ tends to 1 in probability as $t \rightarrow \infty$, uniformly in $N$. More precisely, $\mathbb{E}\left[\left|Z_{t}^{N, i} / m_{t}-1\right|\right] \leq C m_{t}^{-1 / 2}$ for some constant $C$.

3. For any fixed $\ell \geq 1\left(m_{t}^{1 / 2}\left(Z_{t}^{N, i} / m_{t}-1\right)\right)_{i=1, \ldots, \ell}$ goes in law to $\mathcal{N}\left(0, I_{\ell}\right)$ as $(t, N) \rightarrow(\infty, \infty)$ (without condition on the regime).

Point 2 is of course related to the classical law of large numbers for multivariate Hawkes processes; see, for example, Brémaud and Massoulié [9] or [3]. What we prove here is that this law of large numbers is uniform in $N$. From this result, we deduce that $\operatorname{Pr}\left(\left|Z_{t}^{N, i} / m_{t}-1\right|>\varepsilon\right) \leq C \varepsilon^{-1} m_{t}^{-1 / 2}$, uniformly in $N$. In view of the papers by Bordenave and Torrisi [8] and Zhu [48, 49], which concern 
one-dimensional processes, one might expect that a much more fast decay (large deviation bound) could be proved, under a Cramér condition on $\varphi$. It would be interesting to decide if such a bound is uniform in $N$.

THEOREM 11. Work under Assumption 7 with $\varphi$ nonnegative and $h(x)=\mu+$ $x$ for some $\mu>0$. Assume also that $\Lambda=\int_{0}^{\infty} \varphi(s) d s \in(1, \infty]$. Assume finally that $t \mapsto \int_{0}^{t}|d \varphi(s)|$ has at most polynomial growth. For each $N \geq 1$, consider the Hawkes process $\left(Z_{t}^{N, 1}, \ldots, Z_{t}^{N, N}\right)_{t \geq 0}$ with parameters $\left(\mathbb{G}_{N}, \varphi^{N}, \mathbf{h}^{N}\right)$. Consider also the unique solution $\left(m_{t}\right)_{t \geq 0}$ to $(8)$ :

1. We have $m_{t} \sim a_{0} e^{\alpha_{0} t}$ as $t \rightarrow \infty$, where $\alpha_{0}>0$ is determined by $\mathcal{L}_{\varphi}\left(\alpha_{0}\right)=1$ and where $a_{0}=\mu \alpha_{0}^{-2}\left(\int_{0}^{\infty} t \varphi(t) e^{-\alpha_{0} t} d t\right)^{-1}$.

2. For any fixed $i \geq 1, Z_{t}^{N, i} / m_{t}$ tends to 1 in probability as $(t, N) \rightarrow(\infty, \infty)$. More precisely, there is a constant $C$ such that $\mathbb{E}\left[\left|Z_{t}^{N, i} / m_{t}-1\right|\right] \leq m_{t}^{-1 / 2}+$ $C N^{-1 / 2}\left(1+m_{t}^{-1}\right)$.

3. For any fixed $\ell \geq 1$ :

(i) $\left(m_{t}^{1 / 2}\left(Z_{t}^{N, i} / m_{t}-1\right)\right)_{i=1, \ldots, \ell}$ goes in law to $\mathcal{N}\left(0, I_{\ell}\right)$ if $t \rightarrow \infty$ and $N \rightarrow$ $\infty$ with $m_{t} / N \rightarrow 0$;

(ii) $\left(N^{1 / 2}\left(Z_{t}^{N, i} / m_{t}-1\right)\right)_{i=1, \ldots, \ell}$ goes in law to $(X, \ldots, X)$, if $t \rightarrow \infty$ and $N \rightarrow \infty$ with $m_{t} / N \rightarrow \infty$. Here $X$ is a $\mathcal{N}\left(0, \sigma^{2}\right)$-distributed random variable, where $\sigma^{2}=\alpha_{0}^{2} \mu^{-2} \int_{0}^{\infty} e^{-2 \alpha_{0} s} m_{s}^{\prime} d s$.

Let us summarise. At first order (law of large numbers), the mean-field approximation is always good for large times. At second order (central limit theorem), the mean field approximation is always good for large times in the subcritical case, but fails to be relevant for too large times (depending on $N$ ) in the supercritical case: the independence property breaks down.

In the supercritical case, we have the technical condition that $t \mapsto \int_{0}^{t}|d \varphi(s)|$ has at most polynomial growth. This is useful to have some precise estimates of the solution $m$ to (8). This is, for example, always satisfied when $\varphi$ is bounded and nonincreasing, as is often the case in applications. It is slightly restrictive, however, since it forces $\varphi(0)$ to be finite.

It should be possible to study also the critical case, but then the situation is more intricate: many regimes might arise. With a little more work, we could also study, in the supercritical case, the regime where $m_{t} / N \rightarrow x \in(0, \infty)$.

In order to prove Theorems 10 and 11, we will use the following central limit theorem for martingales. For two càdlàg martingales $M$ and $N$, we denote by $[M, N]$ the quadratic covariation defined by $[M, N]_{t}=M_{t} N_{t}-\int_{0}^{t} M_{s-} d N_{s}-$ $\int_{0}^{t} N_{s-} d M_{s}$. When $M$ and $N$ are purely discontinuous, it holds that $[M, N]_{t}=$ $\sum_{s \leq t} \Delta M_{s} \Delta N_{s}$; see Jacod and Shiryaev [24], Chapter I, Section 4e. 
Lemma 12. Let $\ell \geq 1$ be fixed. For $N \geq 1$, consider a family $\left(M_{t}^{N, 1}, \ldots\right.$, $\left.M_{t}^{N, \ell}\right)_{t \geq 0}$ of $\ell$-dimensional local martingales satisfying $M_{0}^{N, i}=0$. Assume that all their jumps are uniformly bounded and that $\left[M^{N, i}, M^{N, j}\right]_{t}=0$ for every $N \geq 1, i \neq j$ and $t \geq 0$. Assume also that there is a continuous increasing function $\left(v_{t}\right)_{t \geq 0}:[0, \infty) \mapsto[0, \infty)$ such that for all $i=1, \ldots, \ell, \lim _{(t, N) \rightarrow(\infty, \infty)} v_{t}^{-2}\left[M^{N, i}\right.$, $\left.M^{N, i}\right]_{t}=1$ in probability. In the case where $v_{\infty}=\lim _{t \rightarrow \infty} v_{t}<\infty$, assume moreover that for all $i=1, \ldots, \ell$, all $t_{0}>0$, uniformly in $t \geq t_{0}, \lim _{N \rightarrow \infty}\left[M^{N, i}\right.$, $\left.M^{N, i}\right]_{t}=v_{t}^{2}$ in probability.

Then $v_{t}^{-1}\left(M_{t}^{N, 1}, \ldots, M_{t}^{N, \ell}\right)$ converges in law to the Gaussian distribution $\mathcal{N}\left(0, I_{\ell}\right)$ as $(t, N) \rightarrow(\infty, \infty)$, where $I_{\ell}$ is the $\ell \times \ell$ identity matrix.

ProOF. Let $\left(t_{N}\right)_{N \geq 1}$ be a sequence of positive numbers such that $t_{N} \rightarrow \infty$. We want to prove that $v_{t_{N}}^{-1}\left(M_{t_{N}}^{N, 1}, \ldots, M_{t_{N}}^{N, \ell}\right)$ converges in law to $\mathcal{N}\left(0, I_{\ell}\right)$. For all $u \in[0,1]$, set

$$
\tau_{u}^{N}=\inf \left\{t \geq 0: v_{t}^{2} \geq u v_{t_{N}}^{2}\right\}
$$

Since $v$ is increasing and continuous, $\tau^{N}$ is also continuous and increasing for each $N$. We also clearly have $v_{\tau_{u}^{N}}^{2}=u v_{t_{N}}^{2}$ for all $u \in[0,1]$ and $\tau_{1}^{N}=t_{N}$. Finally, for each $u>0$ fixed, the sequence $\tau_{u}^{N}$ is increasing.

For all $u \in(0,1], \lim _{N} v_{\tau_{u}^{N}}^{-2}\left[M^{N, i}, M^{N, i}\right]_{\tau_{u}^{N}}=1$ in probability. Indeed, in the case $v_{\infty}=\infty$, this follows from the facts that $\lim _{N} \tau_{u}^{N}=\infty$ and $\lim _{(t, N) \rightarrow(\infty, \infty)} v_{t}^{-2}\left[M^{N, i}, M^{N, i}\right]_{t}=1$. When $v_{\infty}<\infty$, the additional assumption (uniformity in $t \geq t_{0}$ of the convergence as $N \rightarrow \infty$ ) clearly suffices, since the sequence $\tau_{u}^{N}$ is increasing, and thus bounded from below.

We define the martingales $\left(L_{u}^{N, i}\right)_{u \in[0,1]}$ by $L_{u}^{N, i}=v_{t_{N}}^{-1} M_{\tau_{u}^{N}}^{N, i}$. All their jumps are uniformly bounded (because those of $M^{N, i}$ are assumed to be uniformly bounded and because $\sup _{N} v_{t_{N}}^{-1}<\infty$ since $v$ is increasing). We also have $\left[L^{N, i}, L^{N, j}\right]_{u}=0$ for all $i \neq j$, all $u \in[0,1]$. Furthermore, using that $v_{\tau_{u}^{N}}^{2}=u v_{t_{N}}^{2}$,

$$
\left[L^{N, i}, L^{N, i}\right]_{u}=\frac{\left[M^{N, i}, M^{N, i}\right]_{\tau_{u}^{N}}}{v_{t_{N}}^{2}}=\frac{\left[M^{N, i}, M^{N, i}\right]_{\tau_{u}^{N}}}{v_{\tau_{u}^{N}}^{2}} u \rightarrow u
$$

in probability. Therefore, according to Jacod and Shiryaev [24], Theorem VIII3.11 , the process $\left(L_{u}^{N, 1}, \ldots, L_{u}^{N, \ell}\right)_{u \in[0,1]}$ converges in law to $\left(B_{u}^{1}, \ldots, B_{u}^{\ell}\right)_{u \in[0,1]}$ where the $B^{i}$ are independent standard Brownian motions. In particular, $\left(L_{1}^{N, 1}, \ldots, L_{1}^{N, \ell}\right)$ goes in law to $\mathcal{N}\left(0, I_{\ell}\right)$. To complete the proof, it thus suffices to observe that $L_{1}^{N, i}=v_{t_{N}}^{-1} M_{\tau_{1}^{N}}^{N, i}=v_{t_{N}}^{-1} M_{t_{N}}^{N, i}$.

We can now give: 
ProOf OF THEOREM 10. In the present (linear) case, we can rewrite (8) as $m_{t}=\int_{0}^{t}\left(\mu+\int_{0}^{s} \varphi(s-u) d m_{u}\right) d s=\mu t+\int_{0}^{t} \varphi(t-s) m_{s} d s$ by Lemma 22. This equation is studied in details in Lemma 25: recalling that $\Lambda=\int_{0}^{\infty} \varphi(s) d s<1$, we have $m_{t}^{\prime} \sim a_{0}$ and $m_{t} \sim a_{0} t$ as $t \rightarrow \infty$, where $a_{0}=\mu /(1-\Lambda)$, which proves point (i). The proof is now divided in several steps. Step 1 will also be used in the supercritical case.

Step 1. Recall that, for some i.i.d. family $\left(\pi^{i}(d s d z)\right)_{i \geq 1}$ of Poisson measures on $[0, \infty) \times[0, \infty)$ with intensity measure $d s d z$,

$$
Z_{t}^{N, i}=\int_{0}^{t} \int_{0}^{\infty} \mathbf{1}_{\left\{z \leq \mu+N^{-1} \sum_{j=1}^{N} \int_{0}^{s-} \varphi(s-u) d Z_{u}^{N, j}\right\}} \pi^{i}(d s d z) .
$$

We have $\mathbb{E}\left[Z_{t}^{N, i}\right]=m_{t}$. Indeed, by exchangeability, we see that $\mathbb{E}\left[Z_{t}^{N, i}\right]=$ $\mathbb{E}\left[Z_{t}^{N, 1}\right]$ and that

$$
\begin{aligned}
\mathbb{E}\left[Z_{t}^{N, 1}\right] & =\int_{0}^{t}\left(\mu+N^{-1} \sum_{j=1}^{N} \int_{0}^{s} \varphi(s-u) d \mathbb{E}\left[Z_{u}^{N, j}\right]\right) d s \\
& =\int_{0}^{t}\left(\mu+\int_{0}^{s} \varphi(s-u) d \mathbb{E}\left[Z_{u}^{N, 1}\right]\right) d s,
\end{aligned}
$$

whence $\left(\mathbb{E}\left[Z_{t}^{N, 1}\right]\right)_{t \geq 0}$ solves (8), of which the unique solution is $\left(m_{t}\right)_{t \geq 0}$ by Lemma 24.

We next introduce $U_{t}^{N, i}=Z_{t}^{N, i}-m_{t}$ and the martingales [here $\tilde{\pi}^{i}(d s d z)=$ $\left.\pi^{i}(d s d z)-d s d z\right]$

$$
M_{t}^{N, i}=\int_{0}^{t} \int_{0}^{\infty} \mathbf{1}_{\left\{z \leq \mu+N^{-1} \sum_{j=1}^{N} \int_{0}^{s-} \varphi(s-u) d Z_{u}^{N, j}\right\}} \tilde{\pi}^{i}(d s d z) .
$$

We consider the mean processes $\bar{Z}_{t}^{N}=N^{-1} \sum_{1}^{N} Z_{t}^{N, i}, \bar{U}_{t}^{N}=N^{-1} \sum_{1}^{N} U_{t}^{N, i}$ and finally $\bar{M}_{t}^{N}=N^{-1} \sum_{1}^{N} M_{t}^{N, i}$. An easy computation using (8) and Lemma 22 shows that

$$
\begin{aligned}
U_{t}^{N, i} & =M_{t}^{N, i}+\int_{0}^{t} N^{-1} \sum_{j=1}^{N} \int_{0}^{s} \varphi(s-u) d Z_{u}^{N, j} d s-m_{t} \\
& =M_{t}^{N, i}+\int_{0}^{t} \int_{0}^{s} \varphi(s-u)\left(N^{-1} \sum_{j=1}^{N} d Z_{u}^{N, j}-d m_{u}\right) d s \\
& =M_{t}^{N, i}+\int_{0}^{t} \varphi(t-s)\left(N^{-1} \sum_{j=1}^{N} Z_{s}^{N, j}-m_{s}\right) d s,
\end{aligned}
$$

so that

$$
U_{t}^{N, i}=M_{t}^{N, i}+\int_{0}^{t} \varphi(t-s) \bar{U}_{s}^{N} d s
$$


This directly implies that

$$
\bar{U}_{t}^{N}=\bar{M}_{t}^{N}+\int_{0}^{t} \varphi(t-s) \bar{U}_{s}^{N} d s .
$$

Next, we observe that $\left[M^{N, i}, M^{N, j}\right]_{t}=0$ for all $i \neq j$ (because these martingales a.s. never jump simultaneously) and that $\left[M^{N, i}, M^{N, i}\right]_{t}=Z_{t}^{N, i}$. Hence, $\left[\bar{M}^{N}, \bar{M}^{N}\right]_{t}=N^{-1} \bar{Z}_{t}^{N}$. We thus have $\mathbb{E}\left[\left(M_{t}^{N, i}\right)^{2}\right]=\mathbb{E}\left[Z_{t}^{N, i}\right]=m_{t}$ and $\mathbb{E}\left[\left(\bar{M}_{t}^{N}\right)^{2}\right]=N^{-1} \mathbb{E}\left[\bar{Z}_{t}^{N}\right]=N^{-1} m_{t}$.

Step 2. Recalling (16) and using that $\Lambda=\int_{0}^{\infty} \varphi(s) d s<1$, we observe that $\sup _{[0, t]}\left|\bar{U}_{s}^{N}\right| \leq \sup _{[0, t]}\left|\bar{M}_{s}^{N}\right|+\Lambda \sup _{[0, t]}\left|\bar{U}_{s}^{N}\right|$. Consequently,

$$
\mathbb{E}\left[\sup _{[0, t]}\left|\bar{U}_{s}^{N}\right|\right] \leq(1-\Lambda)^{-1} \mathbb{E}\left[\sup _{[0, t]}\left|\bar{M}_{s}^{N}\right|\right] \leq C N^{-1 / 2} m_{t}^{1 / 2}
$$

by the Doob and Cauchy-Schwarz inequalities. We easily deduce that

$$
\mathbb{E}\left[\int_{0}^{t} \varphi(t-s)\left|\bar{U}_{s}^{N}\right| d s\right] \leq \Lambda \mathbb{E}\left[\sup _{[0, t]}\left|\bar{U}_{s}^{N}\right|\right] \leq C N^{-1 / 2} m_{t}^{1 / 2},
$$

whence finally, recalling (15),

$$
m_{t}^{-1} \mathbb{E}\left[\left|U_{t}^{N, i}\right|\right] \leq m_{t}^{-1} \mathbb{E}\left[\left|M_{t}^{N, i}\right|\right]+C m_{t}^{-1} N^{-1 / 2} m_{t}^{1 / 2} \leq C m_{t}^{-1 / 2} .
$$

This says that $\mathbb{E}\left[\left|Z_{t}^{N, i} / m_{t}-1\right|\right] \leq C m_{t}^{-1 / 2}$, and thus proves point 2 .

Step 3. We then fix $\ell \geq 1$ and use (15) to write, for $i=1, \ldots, \ell$,

$$
m_{t}^{1 / 2}\left(Z_{t}^{N, i} / m_{t}-1\right)=m_{t}^{-1 / 2} U_{t}^{N, i}=m_{t}^{-1 / 2} M_{t}^{N, i}+m_{t}^{-1 / 2} \int_{0}^{t} \varphi(t-s) \bar{U}_{s}^{N} d s .
$$

First, $\mathbb{E}\left[m_{t}^{-1 / 2} \int_{0}^{t} \varphi(t-s)\left|\bar{U}_{s}^{N}\right| d s\right] \leq C N^{-1 / 2}$, which tends to 0 as $(t, N) \rightarrow$ $(\infty, \infty)$, by the estimate proved in step 2 . To conclude the proof of point 3 , we thus only have to prove that $\left(m_{t}^{-1 / 2} M_{t}^{N, i}\right)_{i=1, \ldots, \ell}$ goes in law to $\mathcal{N}\left(0, I_{\ell}\right)$ as $(t, N) \rightarrow(\infty, \infty)$. To this end, we apply Lemma 12. The jumps of the martingales $M^{N, i}$ are uniformly bounded (by 1 ) and we have seen that $\left[M^{N, i}, M^{N, j}\right]_{t}=0$ for all $i \neq j$. The function $\left(m_{t}\right)_{t>0}$ is continuous and increases to infinity. It thus suffices to check that, as $(t, N) \rightarrow(\infty, \infty), m_{t}^{-1}\left[M^{N, i}, M^{N, i}\right]_{t} \rightarrow 1$ in probability. Since $\left[M^{N, i}, M^{N, i}\right]_{t}=Z_{t}^{N, i}$, this is an immediate consequence of point 2 .

We now turn to the supercritical case.

Proof OF TheOrem 11. We rewrite (8) as $m_{t}=\int_{0}^{t}\left(\mu+\int_{0}^{s} \varphi(s-\right.$ u) $\left.d m_{u}\right) d s=\mu t+\int_{0}^{t} \varphi(t-s) m_{s} d s$ by Lemma 22. This equation is studied in details in Lemma 26: there is a unique $\alpha_{0}>0$ such that $\mathcal{L}_{\varphi}\left(\alpha_{0}\right)=1$ and, defining $a_{0} \in(0, \infty)$ as in the statement, we have $m_{t} \sim a_{0} e^{\alpha_{0} t}$ and $m_{t}^{\prime} \sim a_{0} \alpha_{0} e^{\alpha_{0} t}$ as $t \rightarrow$ $\infty$, which proves point 1 . We also know that $\Gamma(t)=\sum_{n \geq 1} \varphi^{\star n}(t) \sim\left(a_{0} \alpha_{0}^{2} / \mu\right) e^{\alpha_{0} t}$, that $\Upsilon(t)=\int_{0}^{t} \Gamma(s) d s \sim\left(a_{0} \alpha_{0} / \mu\right) e^{\alpha_{0} t}$ and further properties of $m, m^{\prime}, \Gamma, \Upsilon$ are proved in Lemma 26. 
Step 1. We adopt the same notation as in the proof of Theorem 10, step 1, of which all the results remain valid in the present case. Point 1 follows from Lemma 26(a).

Step 2. First, (16) says exactly that $\bar{U}^{N}=\bar{M}^{N}+\varphi \star \bar{U}^{N}$. Using Lemma 26(e), we deduce that $\bar{U}^{N}=\bar{M}^{N}+\Gamma \star \bar{M}^{N}$ (the processes $\bar{U}^{N}$ and $\bar{M}^{N}$ are clearly a.s. càdlàg, and thus locally bounded). Since $\mathbb{E}\left[\left(\bar{M}_{t}^{N}\right)^{2}\right]=N^{-1} m_{t}$ by step 1 ,

$$
\begin{aligned}
\mathbb{E}\left[\left|\bar{U}_{t}^{N}\right|\right] & \leq \mathbb{E}\left[\left|\bar{M}_{t}^{N}\right|\right]+\int_{0}^{t} \Gamma(t-s) \mathbb{E}\left[\left|\bar{M}_{s}^{N}\right|\right] d s \\
& \leq N^{-1 / 2} m_{t}^{1 / 2}+\int_{0}^{t} \Gamma(t-s) N^{-1 / 2} m_{s}^{1 / 2} d s \\
& \leq C N^{-1 / 2}\left(1+m_{t}\right) .
\end{aligned}
$$

The last inequality easily follows from Lemma 26(b).

Using (16) again, we see that $\int_{0}^{t} \varphi(t-s) \bar{U}_{s}^{N} d s=\bar{U}_{t}^{N}-\bar{M}_{t}^{N}$, whence

$$
\begin{aligned}
\mathbb{E}\left[\left|\int_{0}^{t} \varphi(t-s) \bar{U}_{s}^{N}\right|\right] & \leq \mathbb{E}\left[\left|\bar{M}_{t}^{N}\right|\right]+\mathbb{E}\left[\left|\bar{U}_{t}^{N}\right|\right] \\
& \leq N^{-1 / 2} m_{t}^{1 / 2}+C N^{-1 / 2}\left(1+m_{t}\right) \leq C N^{-1 / 2}\left(1+m_{t}\right) .
\end{aligned}
$$

On the other hand, we know from step 1 that $\mathbb{E}\left[\left(M_{t}^{N, i}\right)^{2}\right]=m_{t}$. Using (15), we conclude that

$$
\mathbb{E}\left[\left|Z_{t}^{N, i} / m_{t}-1\right|\right]=m_{t}^{-1} \mathbb{E}\left[\left|U_{t}^{N, i}\right|\right] \leq m_{t}^{-1 / 2}+C N^{-1 / 2}\left(1+m_{t}^{-1}\right)
$$

which completes the proof of 2 .

Step 3. We then fix $\ell \geq 1$ and write, for $i=1, \ldots, \ell$, by (15),

$$
\left(Z_{t}^{N, i} / m_{t}-1\right)=m_{t}^{-1} U_{t}^{N, i}=m_{t}^{-1} M_{t}^{N, i}+m_{t}^{-1} \int_{0}^{t} \varphi(t-s) \bar{U}_{s}^{N} d s .
$$

Step 3.1. We first consider the regime $(t, N) \rightarrow(\infty, \infty)$ with $m_{t} / N \rightarrow 0$ and study

$$
m_{t}^{1 / 2}\left(Z_{t}^{N, i} / m_{t}-1\right)=m_{t}^{-1 / 2} M_{t}^{N, i}+m_{t}^{-1 / 2} \int_{0}^{t} \varphi(t-s) \bar{U}_{s}^{N} d s .
$$

The second term tends to 0 in probability, because we can bound, using step 2 , its $L^{1}$-norm by $C m_{t}^{-1 / 2} N^{-1 / 2}\left(1+m_{t}\right)$, which tends to 0 in the present regime. We thus just have to prove that $\left(m_{t}^{-1 / 2} M_{t}^{N, i}\right)_{i=1, \ldots, \ell}$ goes in law to $\mathcal{N}\left(0, I_{\ell}\right)$. We use Lemma 12: the martingales $M^{N, i}$ have uniformly bounded (by 1) jumps and we have seen that $\left[M^{N, i}, M^{N, j}\right]_{t}=0$ for $i \neq j$. The function $\left(m_{t}\right)_{t \geq 0}$ is continuous and increases to infinity. It only remains to check that $m_{t}^{-1}\left[M^{N, i}, M^{N, i}\right]_{t}$ tends to 1 in probability. But $\left[M^{N, i}, M^{N, i}\right]_{t}=Z_{t}^{N, i}$, so that the conclusion follows from point 2. 
Step 3.2. We finally consider the regime $(t, N) \rightarrow(\infty, \infty)$ with $m_{t} / N \rightarrow \infty$ and study

$$
N^{1 / 2}\left(Z_{t}^{N, i} / m_{t}-1\right)=N^{1 / 2} m_{t}^{-1} M_{t}^{N, i}+N^{1 / 2} m_{t}^{-1} \int_{0}^{t} \varphi(t-s) \bar{U}_{s}^{N} d s .
$$

First, $N^{1 / 2} m_{t}^{-1} M_{t}^{N, i} \rightarrow 0$ in probability, because its $L^{1}$-norm is bounded by $N^{1 / 2} m_{t}^{-1 / 2}$ (recall that $\mathbb{E}\left[\left(M_{t}^{N, i}\right)^{2}\right]=m_{t}$ ), which tends to 0 in the present regime. Since $V_{t}^{N}:=N^{1 / 2} m_{t}^{-1} \int_{0}^{t} \varphi(t-s) \bar{U}_{s}^{N} d s$ does not depend on $i$, it only remains to prove that $V_{t}^{N}$ goes in law to $\mathcal{N}\left(0, \sigma^{2}\right)$. We write, using (16), recalling that $\bar{U}^{N}=$ $\bar{M}^{N}+\Gamma \star \bar{M}^{N}$ (see step 2) and integrating by parts [recall that $\Upsilon(t)=\int_{0}^{t} \Gamma(s) d s$ ]

$$
\begin{aligned}
V_{t}^{N} & =N^{1 / 2} m_{t}^{-1}\left(\bar{U}_{t}^{N}-\bar{M}_{t}^{N}\right)=N^{1 / 2} m_{t}^{-1} \int_{0}^{t} \Gamma(t-s) \bar{M}_{s}^{N} d s \\
& =N^{1 / 2} m_{t}^{-1} \int_{0}^{t} \Upsilon(t-s) d \bar{M}_{s}^{N} .
\end{aligned}
$$

Introduce $W_{t}^{N}=\left(\alpha_{0} / \mu\right) N^{1 / 2} \int_{0}^{t} e^{-\alpha_{0} s} d \bar{M}_{s}^{N}$ and observe that, since $\mathbb{E}\left[\left[\bar{M}^{N}\right.\right.$, $\left.\left.\bar{M}^{N}\right]_{t}\right]=N^{-1} m_{t}$,

$$
\begin{aligned}
\mathbb{E}\left[\left(V_{t}^{N}-W_{t}^{N}\right)^{2}\right] & =\mathbb{E}\left[N \int_{0}^{t}\left(m_{t}^{-1} \Upsilon(t-s)-\left(\alpha_{0} / \mu\right) e^{-\alpha_{0} s}\right)^{2} d\left[\bar{M}^{N}, \bar{M}^{N}\right]_{s}\right] \\
& =\int_{0}^{t}\left(m_{t}^{-1} \Upsilon(t-s)-\left(\alpha_{0} / \mu\right) e^{-\alpha_{0} s}\right)^{2} m_{s}^{\prime} d s
\end{aligned}
$$

Lemma 26(c) tells us that this tends to 0 as $t \rightarrow \infty$. We thus only have to prove that $W_{t}^{N}$ goes in law to $\mathcal{N}\left(0, \sigma^{2}\right)$ as $(t, N) \rightarrow(\infty, \infty)$.

This follows again from Lemma 12 (with $\ell=1$ ): the jumps of the martingale $\left(W_{t}^{N}\right)_{t \geq 0}$ are bounded by $\left(\alpha_{0} / \mu\right) N^{-1 / 2}$ (because those of $\bar{M}^{N}$ are bounded by $\left.N^{-1}\right)$. The function $v_{t}=\left(\alpha_{0} / \mu\right)\left(\int_{0}^{t} e^{-2 \alpha_{0} s} m_{s}^{\prime} d s\right)^{1 / 2}$ is continuous and increasing to the finite limit $v_{\infty}=\sigma$ (which was defined in the statement). We thus only have to prove that (a) $v_{t}^{-2}\left[W^{N}, W^{N}\right]_{t} \rightarrow 1$ in probability as $(t, N) \rightarrow(\infty, \infty)$, (b) for all $t_{0}>0$, uniformly in $t \geq t_{0}, v_{t}^{-2}\left[W^{N}, W^{N}\right]_{t} \rightarrow 1$ in probability as $N \rightarrow \infty$. By Lemma 12, we will deduce that $v_{t}^{-1} W_{t}^{N}$ goes in law to $\mathcal{N}(0,1)$ as $(t, N) \rightarrow$ $(\infty, \infty)$, which of course implies that $W_{t}^{N}$ goes in law to $\mathcal{N}\left(0, \sigma^{2}\right)$ as desired.

We have, since $\left[\bar{M}^{N}, \bar{M}^{N}\right]_{t}=N^{-1} \bar{Z}_{t}^{N}$,

$$
\left[W^{N}, W^{N}\right]_{t}=\left(\alpha_{0} / \mu\right)^{2} N \int_{0}^{t} e^{-2 \alpha_{0} s} d\left[\bar{M}^{N}, \bar{M}^{N}\right]_{s}=\left(\alpha_{0} / \mu\right)^{2} \int_{0}^{t} e^{-2 \alpha_{0} s} d \bar{Z}_{s}^{N} .
$$

Using that $\bar{Z}_{t}^{N}=\bar{U}_{t}^{N}+m_{t}$ and performing an integration by parts, we see that

$$
\begin{aligned}
{\left[W^{N}, W^{N}\right]_{t} } & =v_{t}^{2}+\left(\alpha_{0} / \mu\right)^{2} \int_{0}^{t} e^{-2 \alpha_{0} s} d \bar{U}_{s}^{N} \\
& =v_{t}^{2}+\left(\alpha_{0} / \mu\right)^{2} e^{-2 \alpha_{0} t} \bar{U}_{t}^{N}+2\left(\alpha_{0}^{3} / \mu^{2}\right) \int_{0}^{t} e^{-2 \alpha_{0} s} \bar{U}_{s}^{N} d s
\end{aligned}
$$


Recalling that $\mathbb{E}\left[\left|\bar{U}_{t}^{N}\right|\right] \leq C N^{-1 / 2}\left(1+m_{t}\right)$, we infer

$$
\begin{aligned}
& \mathbb{E}\left[\left|\left(\alpha_{0} / \mu\right)^{2} e^{-2 \alpha_{0} t} \bar{U}_{t}^{N}+2\left(\alpha_{0}^{3} / \mu^{2}\right) \int_{0}^{t} e^{-2 \alpha_{0} s} \bar{U}_{s}^{N} d s\right|\right] \\
& \leq \frac{C}{N^{1 / 2}}\left(e^{-2 \alpha_{0} t}\left(1+m_{t}\right)+\int_{0}^{t} e^{-2 \alpha_{0} s}\left(1+m_{s}\right) d s\right),
\end{aligned}
$$

which is bounded by $C N^{-1 / 2}$ by Lemma 26 . We have proved that $\sup _{t \geq 0} \mathbb{E}\left[\mid\left[W^{N}\right.\right.$, $\left.\left.W^{N}\right]_{t}-v_{t}^{2} \mid\right] \leq C N^{-1 / 2}$, from which points (a) and (b) above immediately follow. The proof is complete.

4. Nearest neighbour model. We consider here the case where $\mathbb{G}$ is a regular grid, on which particles interact (directly) only if they are neighbours. We will work on $\mathbb{Z}^{d}$, endowed with the set of edges

$$
\mathcal{E}=\left\{(i, j) \in\left(\mathbb{Z}^{d}\right)^{2}:|i-j|=0 \text { or } 1\right\}
$$

where $\left|\left(i_{1}, \ldots, i_{d}\right)\right|=\left(\sum_{r=1}^{d} i_{r}^{2}\right)^{1 / 2}$. Thus, each point has $2 d+1$ neighbours (including itself). We hesitated to include self-interaction, but this avoids some needless complications due to the periodicity of the underlying random walk on $\mathbb{Z}^{d}$.

Assumption 13. (i) The graph $\mathbb{G}=(\mathcal{S}, \mathcal{E})$ is $\mathcal{S}=\mathbb{Z}^{d}$ (for some $d \geq 1$ ) endowed with the above set of edges $\mathcal{E}$.

(ii) There is a nonnegative locally integrable function $\varphi:[0, \infty) \mapsto[0, \infty)$ such that for all $(j, i) \in \mathcal{E}, \varphi_{j i}=(2 d+1)^{-1} \varphi$.

(iii) For all $i \in \mathbb{Z}^{d}$, there is $\mu_{i} \geq 0$ such that $h_{i}(x)=\mu_{i}+x$. The family $\left(\mu_{i}\right)_{i \in \mathbb{Z}^{d}}$ is bounded.

We next introduce some notation. In the whole section, we call vector (and write in bold) a family of numbers indexed by $\mathbb{Z}^{d}$. We call matrix a family indexed by $\mathbb{Z}^{d} \times \mathbb{Z}^{d}$. The identity matrix $I$ is of course defined as $I(i, j)=\mathbf{1}_{\{i=j\}}$. We will often use the product of a matrix and a vector. The matrix $A=(A(i, j))_{i, j \in \mathbb{Z}^{d}}$ defined by

$$
A(i, j)=(2 d+1)^{-1} \mathbf{1}_{\{(i, j) \in \mathcal{E}\}}
$$

will play an important role. Since $A$ is a stochastic matrix, we can define, for any $\Lambda \in(0,1)$,

$$
Q_{\Lambda}(i, j)=\sum_{n \geq 0} \Lambda^{n} A^{n}(i, j)
$$


4.1. Large-time behaviour. Under Assumption 13, we can use Theorem 6 [with $p_{i}=2^{-|i|}$; see Remark 5(ii)]: there is a unique Hawkes process $\left(Z_{t}^{i}\right)_{i \in \mathbb{Z}^{d}, t \geq 0}$ with parameters $(\mathbb{G}, \boldsymbol{\varphi}, \mathbf{h})$ such that $\sum_{i \in \mathbb{Z}^{d}} 2^{-|i|} \mathbb{E}\left[Z_{t}^{i}\right]<\infty$. Let us state the first results of this section. As usual, we treat separately the subcritical and supercritical cases.

THEOREM 14. Work under Assumption 13 and assume further that $\Lambda=$ $\int_{0}^{\infty} \varphi(t) d t<1$. Consider the unique Hawkes process $\left(Z_{t}^{i}\right)_{i \in \mathbb{Z}^{d}, t \geq 0}$ with parameters $(\mathbb{G}, \varphi, \mathbf{h})$. For all $i \in \mathbb{Z}^{d}, t^{-1} Z_{t}^{i}$ goes in probability, as $t \rightarrow \infty$, to $\sum_{j \in \mathbb{Z}^{d}} Q_{\Lambda}(i, j) \mu_{j}$.

THEOREM 15. Work under Assumption 13 and assume further that $\Lambda=$ $\int_{0}^{\infty} \varphi(t) d t \in(1, \infty]$ and that $t \mapsto \int_{0}^{t}|d \varphi(s)|$ has at most polynomial growth. Consider $\alpha_{0}>0$ uniquely defined by $\mathcal{L}_{\varphi}\left(\alpha_{0}\right)=1$. Assume finally that the "mean value"

$$
\mu=\lim _{r \rightarrow \infty} \frac{1}{\#\left\{i \in \mathbb{Z}^{d}:|i| \leq r\right\}} \sum_{|i| \leq r} \mu_{i} \quad \text { exists and is positive. }
$$

Consider the unique Hawkes process $\left(Z_{t}^{i}\right)_{i \in \mathbb{Z}^{d}, t \geq 0}$ with parameters $(\mathbb{G}, \boldsymbol{\varphi}, \mathbf{h})$. Then for all $i \in \mathbb{Z}^{d}, e^{-\alpha_{0} t} Z_{t}^{i}$ goes in probability, as $t \rightarrow \infty$, to $a_{0}=\mu \alpha_{0}^{-2}\left(\int_{0}^{\infty} t \varphi(t) \times\right.$ $\left.e^{-\alpha_{0} t} d t\right)^{-1}$.

Let us comment on these results. In the subcritical case, the parameter $\boldsymbol{\mu}=$ $\left(\mu_{i}\right)_{i \in \mathbb{Z}^{d}}$ is strongly present in the limiting behaviour: the limit of $t^{-1} Z_{t}^{i}$ depends on a certain mean of $\boldsymbol{\mu}$ around the site $i$ and thus depends on $i$. In the supercritical case, the behaviour is very different: the limit value of $e^{-\alpha_{0} t} Z_{t}^{i}$ does not depend on $i$, and depends on $\boldsymbol{\mu}=\left(\mu_{i}\right)_{i \in \mathbb{Z}^{d}}$ only through a global mean value. Observe also that for a finite-dimensional (e.g., scalar) Hawkes process, there is no law of large numbers: one can get a limit of something like $e^{-\alpha_{0} t} Z_{t}^{i}$, but the limit is random; see Zhu [48], Section 5.4 (in particular Theorem 23 and Corollary 1). In that sense, we can say that in the supercritical case, the law of large number is reminiscent of the infinite dimension and of the interaction.

We will need a precise approximation for $A^{n}(i, j)$ where $A$ is defined by (17). It is given by the local central limit theorem, since $A$ is the transition matrix of an aperiodic symmetric random walk on $\mathbb{Z}^{d}$ with bounded jumps. Precisely, we infer from Lawler and Limic ([29], Theorem 2.1.1) and (2.5), that there is a constant $C$ such that for all $n \geq 1$, all $i \in \mathbb{Z}^{d}$,

$$
\left|A^{n}(0, i)-p_{n}(i)\right| \leq \frac{C}{n^{(d+2) / 2}},
$$

where, for $t>0$ and $x \in \mathbb{R}^{d}$,

$$
p_{t}(x)=\left(\frac{2 d+1}{4 \pi t}\right)^{d / 2} \exp \left(-\frac{(2 d+1)|x|^{2}}{4 t}\right) .
$$


To apply [29], Theorem 2.1.1, we needed to compute the covariance matrix $\Gamma$ corresponding to our random walk, we found $\Gamma=2(2 d+1)^{-1} I_{d}, I_{d}$ being the $d \times d$ identity matrix.

LEMma 16. Consider the matrix $(A(i, j))_{i, j \in \mathbb{Z}^{d}}$ defined by (17).

(i) It holds that $\varepsilon_{n}=\sum_{j \in \mathbb{Z}^{d}}\left(A^{n}(i, j)\right)^{2}$ does not depend on $i \in \mathbb{Z}^{d}$ and tends to 0 as $n \rightarrow \infty$.

(ii) Let $\boldsymbol{\mu}=\left(\mu_{i}\right)_{i \in \mathbb{Z}^{d}}$ be bounded and satisfy (19). Then for all $i \in \mathbb{Z}^{d}$, $\lim _{n \rightarrow \infty}\left(A^{n} \boldsymbol{\mu}\right)_{i}=\mu$.

ProOf. In the following, we denote by $C$ a constant depending only on $d$.

Point (i) is easy: since $A^{n}(i, j)=A^{n}(0, j-i)$ and since $A$ is stochastic, one has $\varepsilon_{n}=\sum_{j \in \mathbb{Z}^{d}}\left(A^{n}(0, j)\right)^{2} \leq \sup _{j \in \mathbb{Z}^{d}} A^{n}(0, j)$. Moreover, by $(20), A^{n}(0, j) \leq$ $p_{n}(j)+C n^{-(d+2) / 2} \leq C n^{-d / 2}$. We conclude that $\varepsilon_{n} \leq C n^{-d / 2} \rightarrow 0$ as desired.

Now we turn to the proof of (ii). Let $i \in \mathbb{Z}^{d}$. First, we show that $\lim _{n}\left[\left(A^{n} \boldsymbol{\mu}\right)_{i}-\right.$ $\left.\sum_{j \in \mathbb{Z}^{d}} p_{n}(j) \mu_{j}\right]=0$. Since $\left(A^{n} \boldsymbol{\mu}\right)_{i}=\sum_{j \in \mathbb{Z}^{d}} A^{n}(i, j) \mu_{j}=\sum_{j \in \mathbb{Z}^{d}} A^{n}(0, j-$ $i) \mu_{j}$ and since the family $\left(\mu_{j}\right)_{j \in \mathbb{Z}^{d}}$ is bounded, it suffices to prove that $v_{n}=$ $\sum_{j \in \mathbb{Z}^{d}}\left|A^{n}(0, j-i)-p_{n}(j)\right| \rightarrow 0$. We write

$$
\begin{aligned}
v_{n} & \leq \sum_{|j| \leq n^{1 / 2+1 / 4 d}}\left|A^{n}(0, j-i)-p_{n}(j)\right|+\sum_{|j|>n^{1 / 2+1 / 4 d}}\left(A^{n}(0, j-i)+p_{n}(j)\right) \\
& =v_{n}^{1}+v_{n}^{2}
\end{aligned}
$$

On the one hand, using that $\sum_{j \in \mathbb{Z}^{d}}|j|^{2} A^{n}(0, j) \leq C n$ (the variance of the random walk at time $n$ is of order $n)$, so that $\sum_{j \in \mathbb{Z}^{d}}|j|^{2} A^{n}(0, j-i)=\sum_{k \in \mathbb{Z}^{d}} \mid i+$ $\left.k\right|^{2} A^{n}(0, k) \leq C\left(|i|^{2}+n\right)$, and thus

$$
\begin{aligned}
& \sum_{|j|>n^{1 / 2+1 / 4 d}} A^{n}(0, j-i) \\
& \quad \leq C n^{-1-1 / 2 d} \sum_{j \in \mathbb{Z}^{d}}|j|^{2} A^{n}(0, j-i) \leq C n^{-1-1 / 2 d}\left(|i|^{2}+n\right) .
\end{aligned}
$$

Similarly, we have $\sum_{j \in \mathbb{Z}^{d}}|j|^{2} p_{n}(j) \leq C n$, and thus

$$
\sum_{|j|>n^{1 / 2+1 / 4 d}} p_{n}(j) \leq n^{-1-1 / 2 d} \sum_{j \in \mathbb{Z}^{d}}|j|^{2} p_{n}(j) \leq C n^{-1 / 2 d} .
$$

Consequently, $\lim _{n} v_{n}^{2}=0$. On the other hand,

$$
v_{n}^{1} \leq \sum_{|j| \leq n^{1 / 2+1 / 4 d}}\left|A^{n}(0, j-i)-p_{n}(j-i)\right|+\sum_{|j| \leq n^{1 / 2+1 / 4 d}}\left|p_{n}(j-i)-p_{n}(j)\right| .
$$


From (20), the first sum is bounded by $C n^{-(d+2) / 2} \#\left\{j \in \mathbb{Z}^{d}:|j| \leq n^{1 / 2+1 / 4 d}\right\} \leq$ $C n^{-3 / 4} \rightarrow 0$. For the second sum, we use that, with $c_{d}=(2 d+1) / 4$,

$$
\left|p_{n}(j-i)-p_{n}(j)\right|=p_{n}(j)\left|1-\exp \left(-\frac{c_{d}}{n}|i|^{2}+\frac{2 c_{d}}{n} i . j\right)\right| \text {. }
$$

Hence, for $|j| \leq n^{1 / 2+1 / 4 d}$ and for $n$ large enough (e.g., so that $|i| n^{-1 / 2+1 / 4 d} \leq 1$ ), $\left|p_{n}(j-i)-p_{n}(j)\right| \leq C p_{n}(j)\left(|i|^{2} n^{-1}+|i| n^{-1 / 2+1 / 4 d}\right) \leq C p_{n}(j)\left(1+|i|^{2}\right) n^{-1 / 4}$. Thus, $\sum_{|j| \leq n^{1 / 2+1 / 2 d}}\left|p_{n}(j-i)-p_{n}(j)\right| \leq C\left(1+|i|^{2}\right) n^{-1 / 4}$ and we deduce that $\lim _{n} v_{n}^{1}=0$.

We have shown that $\lim v_{n}=0$. It only remains to check that $\lim _{n} \sum_{j \in \mathbb{Z}^{d}} \mu_{j} \times$ $p_{n}(j)=\mu$. Let $\left(r_{k}\right)_{k \geq 0}$ be the increasing sequence of nonnegative numbers such that $\left\{r_{k}\right\}_{k \geq 0}=\left\{|j|: j \in \mathbb{Z}^{d}\right\}$ and observe that

$$
\sum_{j \in \mathbb{Z}^{d}} \mu_{j} p_{n}(j)=\sum_{k \geq 0} p_{n}\left(r_{k}\right) \sum_{|j|=r_{k}} \mu_{j}
$$

A discrete integration by parts shows that

$$
\begin{aligned}
\sum_{j \in \mathbb{Z}^{d}} \mu_{j} p_{n}(j) & =\sum_{k \geq 0}\left(p_{n}\left(r_{k}\right)-p_{n}\left(r_{k+1}\right)\right) \sum_{|j| \leq r_{k}} \mu_{j} \\
& =\sum_{k \geq 0} v\left(r_{k}\right)\left(p_{n}\left(r_{k}\right)-p_{n}\left(r_{k+1}\right)\right) \frac{1}{v\left(r_{k}\right)} \sum_{|j| \leq r_{k}} \mu_{j},
\end{aligned}
$$

where $v(r)=\#\left\{j \in \mathbb{Z}^{d}:|j| \leq r\right\}$. We easily conclude that $\lim _{n} \sum_{j \in \mathbb{Z}^{d}} \mu_{j} p_{n}(j)=$ $\mu$ as desired, because:

(a) $\lim _{k \rightarrow \infty} \frac{1}{v\left(r_{k}\right)} \sum_{|j| \leq r_{k}} \mu_{j}=\mu$;

(b) for all $k \geq 0$ fixed, $\lim _{n} v\left(r_{k}\right)\left(p_{n}\left(r_{k}\right)-p_{n}\left(r_{k+1}\right)\right)=0$;

(c) $\lim _{n \rightarrow \infty} \sum_{k=0}^{\infty} v\left(r_{k}\right)\left(p_{n}\left(r_{k}\right)-p_{n}\left(r_{k+1}\right)\right)=1$.

Point (a) follows from our condition (19) on $\boldsymbol{\mu}$, point (b) is obvious (because $\left.\left|v\left(r_{k}\right)\left(p_{n}\left(r_{k}\right)-p_{n}\left(r_{k+1}\right)\right)\right| \leq v\left(r_{k}\right) \sup _{i \in \mathbb{Z}^{d}} p_{n}(i) \leq C v\left(r_{k}\right) n^{-d / 2} \rightarrow 0\right)$. To check (c), we write $\sum_{k=0}^{\infty} v\left(r_{k}\right)\left(p_{n}\left(r_{k}\right)-p_{n}\left(r_{k+1}\right)\right)=\sum_{j \in \mathbb{Z}^{d}} p_{n}(j)=\sum_{j \in \mathbb{Z}^{d}} A^{n}(0, j)+$ $\sum_{j \in \mathbb{Z}^{d}}\left[p_{n}(j)-A^{n}(0, j)\right]=1+\sum_{j \in \mathbb{Z}^{d}}\left[p_{n}(j)-A^{n}(0, j)\right]$. This tends to 1 , because $\lim _{n} \sum_{j \in \mathbb{Z}^{d}}\left|p_{n}(j)-A^{n}(0, j)\right|=0$, as seen in the first part of the proof (this is $v_{n}$ in the special case where $i=0$ ).

Let us now give the following.

Proof of TheOREM 14. We split the proof into several steps. We assume that there is at least one $i \in \mathbb{Z}^{d}$ such that $\mu_{i}>0$, because else the result is obvious (because then $Z_{t}^{i}=0$ for all $i \in \mathbb{Z}^{d}$, all $t \geq 0$ ). The first step will also be used in the supercritical case. 
Step 1. We write as usual, for some i.i.d. family $\left(\pi^{i}(d s d z)\right)_{i \geq 1}$ of Poisson measures on $[0, \infty) \times[0, \infty)$ with intensity measure $d s d z$,

$$
Z_{t}^{i}=\int_{0}^{t} \int_{0}^{\infty} \mathbf{1}_{\left\{z \leq \mu_{i}+(2 d+1)^{-1} \sum_{j \rightarrow i} \int_{0}^{s-} \varphi(s-u) d Z_{u}^{j}\right\}} \pi^{i}(d s d z) .
$$

Let us put $m_{t}^{i}=\mathbb{E}\left[Z_{t}^{i}\right]$ and $\mathbf{m}_{t}=\left(m_{t}^{i}\right)_{i \in \mathbb{Z}^{d}}$. A simple computation (using one more time Lemma 22) gives us, for all $i \in \mathbb{Z}^{d}$,

$$
m_{t}^{i}=\mu_{i} t+\int_{0}^{t}(2 d+1)^{-1} \sum_{j \rightarrow i} \varphi(t-s) m_{s}^{j} d s .
$$

Using the vector formalism, this rewrites $\mathbf{m}_{t}=\boldsymbol{\mu} t+\int_{0}^{t} \varphi(t-s)\left(A \mathbf{m}_{s}\right) d s$. We furthermore know (from Theorem 6) that for all $t \geq 0, \sum_{i \in \mathbb{Z}^{d}} 2^{-|i|} m_{t}^{i}<\infty$. Applying Lemma 27, we see that $m^{i}$ is of class $C^{1}$ on $[0, \infty)$ for each $i \in \mathbb{Z}^{d}$, that

$$
\mathbf{m}_{t}^{\prime}=\boldsymbol{\mu}+\int_{0}^{t} \varphi(t-s) A \mathbf{m}_{s}^{\prime} d s
$$

and that

$$
\mathbf{m}_{t}^{\prime}=\left(I+\sum_{n \geq 1} A^{n} \int_{0}^{t} \varphi^{\star n}(s) d s\right) \boldsymbol{\mu} .
$$

Lemma 27 also tells us that $u_{t}=\sup _{i \in \mathbb{Z}^{d}} \sup _{[0, t]}\left(m_{s}^{i}\right)^{\prime}$ is locally bounded, which of course implies that $\sup _{i \in \mathbb{Z}^{d}} \sup _{[0, t]} m_{s}^{i}$ is also locally bounded (because $m_{0}^{i}=0$ for all $i \in \mathbb{Z}^{d}$ ), and that

$$
u_{t} \leq C+\int_{0}^{t} \varphi(t-s) u_{s} d s
$$

We introduce the martingales, for $i \in \mathbb{Z}^{d}$ (we use a tilde for compensation),

$$
M_{t}^{i}=\int_{0}^{t} \int_{0}^{\infty} \mathbf{1}_{\left\{z \leq \mu_{i}+(2 d+1)^{-1} \sum_{j \rightarrow i} \int_{0}^{s-} \varphi(s-u) d Z_{u}^{j}\right\}} \tilde{\pi}^{i}(d s d z)
$$

and observe as usual that $\left[M^{i}, M^{j}\right]_{t}=0$ when $i \neq j$ (because these martingales a.s. never jump at the same time) while $\left[M^{i}, M^{i}\right]_{t}=Z_{t}^{i}$. We finally introduce $U_{t}^{i}=$ $Z_{t}^{i}-m_{t}^{i}$, the vectors $\mathbf{U}_{t}=\left(U_{t}^{i}\right)_{i \in \mathbb{Z}^{d}}$ and $\mathbf{M}_{t}=\left(M_{t}^{i}\right)_{i \in \mathbb{Z}^{d}}$ and observe that

$$
\mathbf{U}_{t}=\mathbf{M}_{t}+\int_{0}^{t} \varphi(t-s) A \mathbf{U}_{s} d s .
$$

Indeed, for every $i \in \mathbb{Z}^{d}$, using Lemma 22 and the equation satisfied by $m_{t}^{i}$, we find

$$
U_{t}^{i}=M_{t}^{i}+\int_{0}^{t}(2 d+1)^{-1} \sum_{j \rightarrow i} \varphi(t-s)\left(Z_{s}^{j}-m_{s}^{j}\right) d s=M_{t}^{i}+\int_{0}^{t} \varphi(t-s)\left(A \mathbf{U}_{s}\right)_{i} d s .
$$


Equation (25) can be solved as usual as

$$
\mathbf{U}_{t}=\left(\mathbf{M}_{t}+\sum_{n \geq 1} \int_{0}^{t} \varphi^{\star n}(t-s) A^{n} \mathbf{M}_{s} d s\right) .
$$

Finally, we easily check that $v_{t}=\sup _{i \in \mathbb{Z}^{d}} \sup _{[0, t]} \mathbb{E}\left[\left|U_{S}^{i}\right|\right]$ is locally bounded (because $\mathbb{E}\left[\left|U_{t}^{i}\right|\right] \leq \mathbb{E}\left[\left|Z_{t}^{i}\right|\right]+m_{t}^{i} \leq 2 m_{t}^{i}$ ) and satisfies (start from (25), use that $\left.\mathbb{E}\left[\left|M_{t}^{i}\right|\right] \leq \mathbb{E}\left[\left[M^{i}, M^{i}\right]_{t}\right]^{1 / 2}=\mathbb{E}\left[Z_{t}^{i}\right]^{1 / 2}=\left(m_{t}^{i}\right)^{1 / 2} \leq\left(\int_{0}^{t} u_{s} d s\right)^{1 / 2}\right)$ and that $A$ is stochastic

$$
v_{t} \leq\left(\int_{0}^{t} u_{s} d s\right)^{1 / 2}+\int_{0}^{t} \varphi(t-s) v_{s} d s .
$$

Step 2. Here, we prove that there is a constant $C$ such that for all $i \in \mathbb{Z}^{d},\left(m_{t}^{i}\right)^{\prime} \leq$ $C$ (and thus also $m_{t}^{i} \leq C t$ ). This follows from (24), which implies that $u_{t} \leq C+$ $\Lambda u_{t}$, whence $u_{t} \leq C /(1-\Lambda)$.

Step 3. For all $i \in \mathbb{Z}^{d},\left(m_{t}^{i}\right)^{\prime} \sim\left(Q_{\Lambda} \boldsymbol{\mu}\right)_{i}$, whence also $m_{t}^{i} \sim\left(Q_{\Lambda} \boldsymbol{\mu}\right)_{i} t$, as $t \rightarrow$ $\infty$. Indeed, starting from (23), using the monotone convergence theorem and that $\int_{0}^{\infty} \varphi^{\star n}(s) d s=\left(\int_{0}^{\infty} \varphi(s) d s\right)^{n}=\Lambda^{n}$,

$$
\lim _{t \rightarrow \infty}\left(m_{t}^{i}\right)^{\prime}=\left(\left(I+\sum_{n \geq 1} \Lambda^{n} A^{n}\right) \boldsymbol{\mu}\right)_{i}=\left(Q_{\Lambda} \boldsymbol{\mu}\right)_{i} .
$$

Step 4. There is a constant $C$ such that for all $i \in \mathbb{Z}^{d}$, all $t \geq 0, \mathbb{E}\left[\left|U_{t}^{i}\right|\right] \leq C t^{1 / 2}$. Indeed, this follows from (27) and step 2, which imply that $v_{t} \leq C t^{1 / 2}+\Lambda v_{t}$, whence $v_{t} \leq C t^{1 / 2} /(1-\Lambda)$.

Step 5. The conclusion follows immediately, writing

$$
\mathbb{E}\left[\left|\frac{Z_{t}^{i}}{t}-\left(Q_{\Lambda} \boldsymbol{\mu}\right)_{i}\right|\right] \leq \mathbb{E}\left[\left|\frac{U_{t}^{i}}{t}\right|\right]+\left|\frac{m_{t}^{i}}{t}-\left(Q_{\Lambda} \boldsymbol{\mu}\right)_{i}\right|,
$$

which tends to 0 as $t \rightarrow \infty$ by steps 3 and 4 .

We now turn to the supercritical case.

Proof OF THEOREM 15. We consider $m$ (not to be confused with m) the unique solution to $m_{t}=\mu t+\int_{0}^{t} \varphi(t-s) m_{s} d s$, where $\mu$ is the mean value defined by (19). This equation is studied in details in Lemma 26: with $\alpha_{0}$ and $a_{0}$ defined in the statement, we have $m_{t} \sim a_{0} e^{\alpha_{0} t}$ and $m_{t}^{\prime} \sim a_{0} \alpha_{0} e^{\alpha_{0} t}$ as $t \rightarrow \infty$, as well as $\Gamma(t)=\sum_{n \geq 1} \varphi^{\star n}(t) \sim\left(a_{0} \alpha_{0}^{2} / \mu\right) e^{\alpha_{0} t}$ and $\Upsilon(t)=\int_{0}^{t} \Gamma(s) d s \sim\left(a_{0} \alpha_{0} / \mu\right) e^{\alpha_{0} t}$.

Step 1. We adopt the notation introduced in step 1 of the proof of Theorem 14.

Step 2. Here, we check that there is $C$ such that for all $i \in \mathbb{Z}^{d},\left(m_{t}^{i}\right)^{\prime} \leq$ $C e^{\alpha_{0} t}$ (and thus $m_{t}^{i} \leq C e^{\alpha_{0} t}$ ). This follows from (24), which tells us that $u_{t}=$ $\sup _{i \in \mathbb{Z}^{d}}\left(m_{t}^{i}\right)^{\prime}$ is locally bounded and satisfies $u_{t} \leq C+\int_{0}^{t} \varphi(t-s) u_{s} d s$. Setting $h_{t}=u_{t}-\int_{0}^{t} \varphi(t-s) u_{s} d s$, we see that $h_{t}$ is locally bounded (from above and from 
below), because $u$ is locally bounded and $\varphi$ is locally integrable. We furthermore have $u=h+u \star \varphi$. Applying Lemma 26(e), we deduce that $u=h+h \star \Gamma$. But $h$ is bounded from above by $C$. Consequently, $u \leq C+C \star \Gamma=C(1+\Upsilon)$, where $\Upsilon$ was defined in Lemma 26. The conclusion follows from Lemma 26(b).

Step 3. We now show that for all $i \in \mathbb{Z}^{d},\left(m_{t}^{i}\right)^{\prime} \sim\left(m_{t}\right)^{\prime}$ (whence $m_{t}^{i} \sim m_{t}$ ) as $t \rightarrow \infty$. Let us fix $i \in \mathbb{Z}^{d}$ and set $r_{t}^{i}=\left(m_{t}^{i}\right)^{\prime}-\left(m_{t}\right)^{\prime}$, which satisfies $r_{t}^{i}=$ $\mu_{i}-\mu+\sum_{n \geq 1}\left(\left(A^{n} \boldsymbol{\mu}\right)_{i}-\mu\right) \int_{0}^{t} \varphi^{\star n}(s) d s$. We know from Lemma 16(ii) that $\eta_{n}=\left(A^{n} \boldsymbol{\mu}\right)_{i}-\mu$ tends to 0 as $n \rightarrow \infty$. Consequently, Lemma 26(d) tells us that $e^{-\alpha_{0} t} \sum_{n \geq 1}\left(\left(A^{n} \boldsymbol{\mu}\right)_{i}-\mu\right) \int_{0}^{t} \varphi^{\star n}(s) d s$ tends to 0 as $t \rightarrow \infty$. Hence, $e^{-\alpha_{0} t} r_{t}^{i} \rightarrow 0$ as $t \rightarrow \infty$. Using finally that $m_{t}^{\prime} \sim a_{0} \alpha_{0} e^{\alpha_{0} t}$ as $t \rightarrow \infty$, we conclude that $\left(m_{t}^{i}\right)^{\prime} / m_{t}^{\prime}=$ $1+r_{t}^{i} / m_{t}^{\prime} \sim 1+\left(a_{0} \alpha_{0}\right)^{-1} e^{-\alpha_{0} t} r_{t}^{i} \rightarrow 1$ as desired.

Step 4 . Here, we check that for every $i \in \mathbb{Z}^{d}, e^{-\alpha_{0} t} \mathbb{E}\left[\left|U_{t}^{i}\right|\right]$ tends to 0 as $t \rightarrow \infty$. We start from (26) to write

$$
\left|U_{t}^{i}\right| \leq\left|M_{t}^{i}\right|+\sum_{n \geq 1} \int_{0}^{t} \varphi^{\star n}(t-s)\left|\left(A^{n} \mathbf{M}_{s}\right)_{i}\right| d s .
$$

But $\mathbb{E}\left[\left|M_{t}^{i}\right|\right] \leq \mathbb{E}\left[Z_{t}^{i}\right]^{1 / 2}=\left(m_{t}^{i}\right)^{1 / 2} \leq C e^{\alpha_{0} t / 2}$ by step 2 and $\mathbb{E}\left[\left(A^{n} \mathbf{M}_{t}\right)_{i}^{2}\right]=$ $\sum_{j}\left(A^{n}(i, j)\right)^{2} m_{s}^{j} \leq C e^{\alpha_{0} t} \sum_{j}\left(A^{n}(i, j)\right)^{2} \leq C e^{\alpha_{0} t} \varepsilon_{n}$ by Lemma 16(i), with $\varepsilon_{n} \rightarrow$ 0 as $n \rightarrow \infty$. Consequently,

$$
e^{-\alpha_{0} t} \mathbb{E}\left[\left|U_{t}^{i}\right|\right] \leq C e^{-\alpha_{0} t / 2}+C e^{-\alpha_{0} t} \sum_{n \geq 1} \varepsilon_{n}^{1 / 2} \int_{0}^{t} \varphi^{\star n}(t-s) e^{\alpha_{0} s / 2} d s .
$$

Lemma 26(d) allows us to conclude.

Step 5. The conclusion follows, writing

$$
\mathbb{E}\left[\left|\frac{Z_{t}^{i}}{a_{0} e^{\alpha_{0} t}}-1\right|\right] \leq \mathbb{E}\left[\left|\frac{U_{t}^{i}}{a_{0} e^{\alpha_{0} t}}\right|\right]+\left|\frac{m_{t}^{i}}{a_{0} e^{\alpha_{0} t}}-1\right|,
$$

and using steps 3, 4 and that $m_{t} \sim a_{0} e^{\alpha_{0} t}$ by Lemma 26(a).

4.2. Study of an impulsion. Here, we want to study how an impulsion at time 0 at $i=0$ propagates. To this end, we work under Assumption 13 with $\mu_{i}=0$ for all $i \in \mathbb{Z}^{d}$, but we assume that $Z^{0}$ has a jump at time 0 . Such a study is of course important: it allows us to measure, in some sense, the range of the interaction.

We first define precisely the process under study.

DEFINITION 17. We work under Assumption 13(i)-(ii) and consider a family $\left(\pi^{i}(d s d z), i \in \mathbb{Z}^{d}\right)$ of i.i.d. $\left(\mathcal{F}_{t}\right)_{t \geq 0}$-Poisson measures on $[0, \infty) \times[0, \infty)$ with intensity measure $d s d z$. We say that a family $\left(Z_{t}^{i}\right)_{i \in \mathbb{Z}^{d}, t \geq 0}$ of $\left(\mathcal{F}_{t}\right)_{t \geq 0}$-adapted counting processes is an impulsion Hawkes process if

$$
\forall i \in \mathbb{Z}^{d}, \quad Z_{t}^{i}=\int_{0}^{t} \int_{0}^{\infty} \mathbf{1}_{\left\{z \leq \sum_{j \rightarrow i}(2 d+1)^{-1}\left[\int_{0}^{s-} \varphi(s-u) d Z_{u}^{j}+\varphi(s) \mathbf{1}_{\{j=0\}}\right]\right\}} \pi^{i}(d s d z) .
$$


As said previously, the term $\sum_{j \rightarrow i} \varphi(s) \mathbf{1}_{\{j=0\}}$ is interpreted as an excitation due to a forced jump of $Z^{0}$ at time 0: simply rewrite it as $\mathbf{1}_{\{0 \rightarrow i\}} \int_{0}^{s-} \varphi(s-u) \delta_{u}(d s)$.

The following proposition is easy.

Proposition 18. Adopt the assumptions and notation of Definition 17. There exists a pathwise unique impulsion Hawkes process $\left(Z_{t}^{i}\right)_{i \in \mathbb{Z}^{d}, t \geq 0}$ such that $\sum_{i \in \mathbb{Z}^{d}} \mathbb{E}\left[Z_{t}^{i}\right]<\infty$ for all $t \geq 0$.

Exactly as Theorem 6, this result can be deduced from Massoulié [32], Theorem 2 , in the subcritical case where $\int_{0}^{\infty} \varphi(s) d s<1$.

Proof OF Proposition 18. The proof resembles much that of Theorem 6, so we only sketch it. We start with uniqueness and thus consider two impulsion Hawkes processes $\left(Z_{t}^{i}\right)_{i \in \mathbb{Z}^{d}, t \geq 0}$ and $\left(\widetilde{Z}_{t}^{i}\right)_{i \in \mathbb{Z}^{d}, t \geq 0}$ such that $\sum_{i \in \mathbb{Z}^{d}} \mathbb{E}\left[Z_{t}^{i}+\widetilde{Z}_{t}^{i}\right]<$ $\infty$. We set $\Delta_{t}^{i}=\int_{0}^{t}\left|d\left(Z_{s}^{i}-\widetilde{Z}_{s}^{i}\right)\right|, \delta_{t}^{i}=\mathbb{E}\left[\Delta_{t}^{i}\right]$ and $\delta_{t}=\sum_{i \in \mathbb{Z}^{d}} \delta_{t}^{i}$ (which is locally bounded by assumption). We may check that $\delta_{t}^{i} \leq(2 d+1)^{-1} \int_{0}^{t} \sum_{j \rightarrow i} \varphi(t-$ $s) \delta_{s}^{j} d s$ exactly as in the proof of Theorem 6. Summing in $i$ and recalling that each site has $2 d+1$ neighbours, we find $\delta_{t} \leq \int_{0}^{t} \varphi(t-s) \delta_{s} d s$. Lemma 23(i) tells us that $\delta_{t}=0$ for all $t$, whence pathwise uniqueness.

Existence follows from a Picard iteration. Let us only check an $a$ priori estimate implying that $\sum_{i \in \mathbb{Z}^{d}} \mathbb{E}\left[Z_{t}^{i}\right]<\infty$ for all $t \geq 0$. Set $m_{t}^{i}=\mathbb{E}\left[Z_{t}^{i}\right]$ and $m_{t}=\sum_{i \in \mathbb{Z}^{d}} m_{t}^{i}$. A direct computation using Lemma 22 shows that $m_{t}^{i}=(2 d+1)^{-1} \sum_{j \rightarrow i}\left[\int_{0}^{t} \varphi(t-\right.$ $\left.s) m_{s}^{j} d s+\mathbf{1}_{\{j=0\}} \int_{0}^{t} \varphi(s) d s\right]$. Summing in $i$, we find $m_{t}=\int_{0}^{t} \varphi(t-s) m_{s} d s+$ $\int_{0}^{t} \varphi(s) d s$. Using Lemma 23(i), that $\varphi$ is locally integrable [and that $\int_{0}^{t} \varphi(s) d s$ is locally bounded], we deduce that $\sup _{[0, T]} m_{t} \leq C(T, \varphi)$ as desired.

We next compute the probability of the extinction event. Point 1 is a noticeable property that makes the result very easy and precise.

THEOREM 19. Adopt the assumptions and notation of Definition 17 and consider the impulsion Hawkes process $\left(Z_{t}^{i}\right)_{i, \in \mathbb{Z}^{d}, t \geq 0}$.

1. The process $Z_{t}=\sum_{i \in \mathbb{Z}} Z_{t}^{i}$ is a scalar impulsion Hawkes process with excitation function $\varphi$. In other words, $\left(Z_{t}\right)_{t \geq 0}$ is a counting process with compensator $A_{t}=\int_{0}^{t} \lambda_{s} d s$, where

$$
\lambda_{t}=\varphi(t)+\int_{0}^{t-} \varphi(t-s) d Z_{s}
$$

2. We introduce the extinction event defined by $\Omega_{e}=\left\{\lim _{t \rightarrow \infty} \sum_{i \in \mathbb{Z}^{d}} Z_{t}^{i}<\right.$ $\infty$ \}. Setting $\Lambda=\int_{0}^{\infty} \varphi(s) d s$, we have (i) $\operatorname{Pr}\left(\Omega_{e}\right)=0$ if $\Lambda=\infty$; (ii) $\operatorname{Pr}\left(\Omega_{e}\right)=$ $\exp \left(-\gamma_{\Lambda} \Lambda\right)$ if $\Lambda \in(1, \infty)$, where $\gamma_{\Lambda} \in(0,1)$ is characterised by $\gamma_{\Lambda} \Lambda+\log (1-$ $\left.\gamma_{\Lambda}\right)=0$; (iii) $\operatorname{Pr}\left(\Omega_{e}\right)=1$ if $\Lambda \in(0,1]$. 
Of course, we can sometimes use this theorem, by a simple comparison argument, if $\varphi_{j i}$ depends on $i, j$. For example, to guarantee nonextinction with probability one, it suffices that all the $\varphi_{j i}$ are bounded below by some $(2 d+1)^{-1} \varphi$ such that $\int_{0}^{\infty} \varphi(s) d s=\infty$.

When $\Lambda \in(0,1)$, a detailed study of the tail distribution of the extinction time is handled by Brémaud, Nappo and Torrisi [10], for a more general scalar model with marks.

ProOf OF THEOREM 19. Point 1 is immediate: the compensator of the counting process $\left(Z_{t}\right)_{t \geq 0}$ is

$$
\begin{aligned}
A_{t} & =\int_{0}^{t} \sum_{i \in \mathbb{Z}^{d}} \sum_{j \rightarrow i}(2 d+1)^{-1}\left[\int_{0}^{s-} \varphi(s-u) d Z_{u}^{j}+\varphi(s) \mathbf{1}_{\{j=0\}}\right] d s \\
& =\int_{0}^{t} \sum_{j \in \mathbb{Z}^{d}}\left[\int_{0}^{s-} \varphi(s-u) d Z_{u}^{j}+\varphi(s) \mathbf{1}_{\{j=0\}}\right] d s \\
& =\int_{0}^{t}\left[\varphi(s)+\int_{0}^{s-} \varphi(s-u) d Z_{u}\right] d s .
\end{aligned}
$$

We next prove point 2. It is well-known folklore that a scalar impulsion Hawkes process can be related to a Poisson Galton-Watson process with Poisson $(\Lambda)$ reproduction law, but we give a direct proof for the sake of completeness. If $\Lambda=\infty$, it suffices to note that $\operatorname{Pr}\left(\Omega_{e}\right)=\operatorname{Pr}\left(Z_{\infty}<\infty\right)=\operatorname{Pr}\left(A_{\infty}<\infty\right) \leq \operatorname{Pr}\left(\int_{0}^{\infty} \varphi(s) d s<\right.$ $\infty)=0$. When $\Lambda<\infty$, we introduce the martingale, for $\gamma \in(0,1), N_{t}^{\gamma}=$ $-\gamma\left(Z_{t}-A_{t}\right)=-\gamma Z_{t}+\gamma \int_{0}^{t} \varphi(s) d s+\gamma \int_{0}^{t} \varphi(t-s) Z_{s} d s$ by Lemma 22. We denote by $M_{t}^{\gamma}=\mathcal{E}\left(N^{\gamma}\right)_{t}=e^{\gamma A_{t}} \prod_{s \leq t}\left(1-\gamma \Delta Z_{s}\right)$ its Doléans-Dade exponential; see Jacod and Shiryaev [24], Chapter 1 , Section 4 f. Since $Z$ is a counting process, we see that

$$
M_{t}^{\gamma}=\exp \left(\gamma \int_{0}^{t} \varphi(s) d s+\gamma \int_{0}^{t} \varphi(t-s) Z_{s} d s+\log (1-\gamma) Z_{t}\right) .
$$

If $\gamma \Lambda+\log (1-\gamma) \leq 0$ then $M^{\gamma}$ is bounded [because $\gamma \int_{0}^{t} \varphi(s) d s+\gamma \int_{0}^{t} \varphi(t-$ s) $\left.Z_{s} d s+\log (1-\gamma) Z_{t} \leq \gamma \Lambda+(\gamma \Lambda+\log (1-\gamma)) Z_{t} \leq \gamma \Lambda\right]$, and thus converges in $L^{1}$. Consequently, $\mathbb{E}\left[M_{\infty}^{\gamma}\right]=1$ and [one easily verifies, using that $Z$ is nondecreasing, that $\left.\lim _{t \rightarrow \infty} \int_{0}^{t} \varphi(t-s) Z_{s} d s=\Lambda Z_{\infty}\right]$

$$
\mathbb{E}\left[\exp \left(\gamma \Lambda+(\log (1-\gamma)+\gamma \Lambda) Z_{\infty}\right)\right]=1 \text {. }
$$

But for all $x>0$, there is a unique $\gamma(x) \in(0,1)$ such that $\gamma(x) \Lambda+\log (1-$ $\gamma(x))=-x$, whence

$$
\mathbb{E}\left[\exp \left(-x Z_{\infty}\right)\right]=\exp (-\Lambda \gamma(x))
$$

Consequently,

$$
\operatorname{Pr}\left(\Omega_{e}\right)=\operatorname{Pr}\left(Z_{\infty}<\infty\right)=\lim _{x \rightarrow 0+} \mathbb{E}\left[\exp \left(-x Z_{\infty}\right)\right]=\exp (-\Lambda \gamma(0+))
$$


If $\Lambda \in(0,1]$, we see that $\gamma(0+)=0$, so that $\operatorname{Pr}\left(\Omega_{e}\right)=1$. If now $\Lambda>1, \gamma(0+)$ is the unique solution in $(0,1)$ to $\gamma(0+) \Lambda+\log (1-\gamma(0+))=0$.

We next study more deeply, in the super-critical case, how the impulsion propagates. Unfortunately, the computations are really tedious: we decided to restrict ourselves to a particular case ( $\varphi$ is an exponential function) where some computations are explicit. We believe that the result below can be extended to a general class of functions $\varphi$, but a difficult technical lemma is required.

THEOREM 20. Work under Assumption 13(i)-(ii), with $\varphi(t)=a e^{-b t}$, for some $a>b>0$. Consider the impulsion Hawkes process $\left(Z_{t}^{i}\right)_{i \in \mathbb{Z}^{d}, t>0}$. Since $\int_{0}^{\infty} \varphi(s) d s=a / b>1$, we know from Theorem 19 that $\operatorname{Pr}\left(\Omega_{e}\right) \in(0,1)$. We set $\alpha_{0}=a-b\left[\right.$ for which $\left.\mathcal{L}_{\varphi}\left(\alpha_{0}\right)=1\right]$ and we recall that the Gaussian density $p_{t}(x)$ is defined by (21).

(i) There are some constants $C>0$ and $t_{0}>0$ and a random variable $H \geq 0$ such that for all $i \in \mathbb{Z}^{d}$, all $t \geq t_{0}$,

$$
\mathbb{E}\left[\left|Z_{t}^{i}-H p_{a t}(i) e^{\alpha_{0} t}\right|\right] \leq \frac{C e^{\alpha_{0} t}}{t^{d / 2+1 / 3}} .
$$

(ii) For all $x \in \mathbb{R}^{d}, t^{d / 2} e^{-\alpha_{0} t} Z_{t}^{\left\lfloor x t^{1 / 2}\right\rfloor} \rightarrow H p_{a}(x)$ in probability as $t \rightarrow \infty$. Here, we define the "integer part" $\lfloor y\rfloor$ of $y=\left(y_{1}, \ldots, y_{d}\right) \in \mathbb{R}^{d}$, by $\lfloor y\rfloor=$ $\left(\left\lfloor y_{1}\right\rfloor, \ldots,\left\lfloor y_{d}\right\rfloor\right) \in \mathbb{Z}^{d}$.

(iii) The random variable $H$ is positive on the event $\Omega_{e}^{c}$.

(iv) Actually, $H=\lim _{t \rightarrow \infty} e^{-\alpha_{0} t} \sum_{i \in \mathbb{Z}^{d}} Z_{t}^{i}$ and $H=\lim _{t \rightarrow \infty}(4 \pi t /(2 d+$ 1)) ${ }^{d / 2} e^{-\alpha_{0} t} Z_{t}^{0}$ in $L^{1}$.

This result describes quite precisely how an impulsion propagates. Conditional on nonextinction, the process $\left(Z_{t}^{i}\right)_{i \in \mathbb{Z}^{d}}$ resembles a Gaussian profile, with height $t^{-d / 2} H e^{\alpha_{0} t}$ and radius $\sqrt{t}$, for some positive random variable $H$.

Compared to the previous result (Theorem 15), the growth is only very slightly slower: a single impulsion at the site 0 produces a growth in $t^{-d / 2} e^{\alpha_{0} t}$, while we have $e^{\alpha_{0} t}$ when all the sites are regularly excited (as is, e.g., the case when $\mu_{i}=1$ for all $i$ ).

It is important to note that, even if the growth "near 0" of the process is very fast (exponential), the spatial propagation is quite slow (of order $\sqrt{t}$ ).

Since $H=\lim _{t \rightarrow \infty} e^{-\alpha_{0} t} Z_{t}$, where $Z_{t}:=\sum_{i \in \mathbb{Z}^{d}} Z_{t}^{i}$ is nothing but a scalar impulsion Hawkes process by Theorem 19, much more information on the distribution of $H$ can be found in the papers by Hawkes [19] and Lewis [30]. See also the work of Torrisi and Leonardi [44] for related modelling purposes.

It is likely that these results could be proved for more general functions $\varphi$. Since we assume that $\varphi$ is an exponential function, the couple (Hawkes process, intensity process) is actually a Markov process. See Oakes [33] in the scalar case. But we do 
absolutely not use the Markov property. What we use is that in Lemma 21 below, many explicit computations are possible, that considerably simplifies the study.

We start with some preliminary computations.

LEMMA 21. Adopt the notation and assumptions of Theorem 20. Introduce also, for $i \in \mathbb{Z}^{d}$ and $t \geq 0, \Gamma(i, t)=\sum_{n \geq 1} A^{n}(0, i) \varphi^{\star n}(t)$.

(i) For all $n \geq 1$, all $t \geq 0, \varphi^{\star n}(t)=(\text { at })^{n-1} e^{-b t} /(n-1)$ !.

(ii) For all $t \geq 0, \sum_{i \in \mathbb{Z}^{d}} \Gamma(i, t)=e^{\alpha_{0} t}$.

(iii) There is $\bar{C}$ such that for all $t \geq 0, \sum_{i \in \mathbb{Z}^{d}}|i|^{2} \Gamma(i, t) \leq C(1+t) e^{\alpha_{0} t}$.

(iv) There are some constants $C$ and $t_{0}>0$ such that for all $t \geq t_{0}$, all $i \in \mathbb{Z}^{d}$,

$$
\begin{aligned}
\left|\Gamma(i, t)-p_{a t}(i) e^{\alpha_{0} t}\right| & \leq \frac{C e^{\alpha_{0} t}}{t^{d / 2+1 / 3}}, \\
\left|\int_{0}^{t} \Gamma(i, s) d s-\frac{1}{\alpha_{0}} p_{a t}(i) e^{\alpha_{0} t}\right| & \leq \frac{C e^{\alpha_{0} t}}{t^{d / 2+1 / 3}} .
\end{aligned}
$$

PROOF. Point (i) is well known and can be checked recursively. Using that $A^{n}$ is a stochastic matrix, we see that $\sum_{i \in \mathbb{Z}^{d}} \Gamma(i, t)=\sum_{n \geq 1} \varphi^{\star n}(t)$. Hence, (ii) follows from (i).

Next, we recall that $A$ is the transition matrix of a symmetric random walk on $\mathbb{Z}^{d}$ (with bounded jumps), so that there is a constant $C$ such that for all $n \geq 0$, $\sum_{i \in \mathbb{Z}^{d}}|i|^{2} A^{n}(0, i) \leq C n$ (its variance at time $n$ is of order $n$ ). Consequently,

$$
\begin{aligned}
\sum_{i \in \mathbb{Z}^{d}}|i|^{2} \Gamma(i, t) & \leq C \sum_{n \geq 1} n \varphi^{\star n}(t)=C \sum_{n \geq 1} \frac{n(a t)^{n-1} e^{-b t}}{(n-1) !} \\
& =C\left[\sum_{n \geq 1} \frac{(a t)^{n-1} e^{-b t}}{(n-1) !}+\sum_{n \geq 2} \frac{(a t)^{n-1} e^{-b t}}{(n-2) !}\right] .
\end{aligned}
$$

This is easily computed: it gives $C e^{\alpha_{0} t}[1+a t]$.

Point (iv) is more complicated. First, we need the Gaussian approximation of $A^{n}(0, i)$ given by (20). We will also need the following result, which can be found, for example, in [14], Lemma 9(d) [plus the fact that for $x \in(0,1),(x+1) \log (x+$ $\left.1)-x \geq x^{2} / 4\right]$ : for any $\lambda>0$, for $X$ a Poisson $(\lambda)$-distributed random variable, for any $x \in(0,1)$,

$$
\operatorname{Pr}(|X-\lambda| \geq \lambda x) \leq 2 \exp \left(-\lambda x^{2} / 4\right) .
$$

We now turn to our problem. Observing that $\sum_{n \geq 1} \varphi^{\star n}(t)=e^{\alpha_{0} t}$, we write

$$
\Delta(i, t)=\left|\Gamma(i, t)-p_{a t}(i) e^{\alpha_{0} t}\right|=\left|\sum_{n \geq 1} \varphi^{\star n}(t)\left(A^{n}(0, i)-p_{a t}(i)\right)\right| .
$$


We now assume that $t$ is large enough so that $p_{t / a}(i) \leq 1$ for all $i$ and we write

$$
\begin{aligned}
\Delta(i, t) \leq & \sum_{n \geq 1} \varphi^{\star n}(t) \mathbf{1}_{\left\{|(n-1)-a t|>(a t)^{2 / 3}\right\}} \\
& +\sum_{n \geq 1} \varphi^{\star n}(t) \mathbf{1}_{\left\{|(n-1)-a t| \leq(a t)^{2 / 3}\right\}}\left|A^{n}(0, i)-p_{n}(i)\right| \\
& +\sum_{n \geq 1} \varphi^{\star n}(t) \mathbf{1}_{\left\{|(n-1)-a t| \leq(a t)^{2 / 3}\right\}}\left|p_{n}(i)-p_{a t}(i)\right| \\
= & \Delta_{1}(i, t)+\Delta_{2}(i, t)+\Delta_{3}(i, t) .
\end{aligned}
$$

First, using point (i) and (30) [with $\lambda=a t$ and $x=(a t)^{-1 / 3}$ ], we see that (if $t$ is large enough so that $a t>1$ )

$$
\Delta_{1}(i, t) \leq e^{\alpha_{0} t} \sum_{n \geq 1} e^{-a t} \frac{(a t)^{n-1}}{(n-1) !} \mathbf{1}_{\left\{|(n-1)-a t|>(a t)^{2 / 3}\right\}} \leq 2 e^{\alpha_{0} t} e^{-(a t)^{1 / 3} / 4} .
$$

We next use (20) and assume that $t$ is large enough so that $|(n-1)-a t| \leq(a t)^{2 / 3}$ implies $n \geq a t / 2$ :

$$
\begin{aligned}
\Delta_{2}(i, t) & \leq C \sum_{n \geq 1} n^{-(d+2) / 2} \varphi^{\star n}(t) \mathbf{1}_{\left\{|(n-1)-a t| \leq(a t)^{2 / 3}\right\}} \\
& \leq C(a t)^{-(d+2) / 2} \sum_{n \geq 1} \varphi^{\star n}(t) \leq C t^{-(d+2) / 2} e^{\alpha_{0} t} .
\end{aligned}
$$

Finally, we observe that $\left|\partial_{t} p_{t}(x)\right| \leq C t^{-d / 2-1}$, so that, if $t$ is sufficiently large, $|(n-1)-a t| \leq(a t)^{2 / 3}$ implies $\left|p_{n}(i)-p_{a t}(i)\right| \leq C t^{-d / 2-1 / 3}$. Consequently,

$$
\Delta_{3}(i, t) \leq C t^{-d / 2-1 / 3} \sum_{n \geq 1} \varphi^{\star n}(t) \leq C t^{-d / 2-1 / 3} e^{\alpha_{0} t} .
$$

We have proved that there are $C$ and $t_{0}$ such that for all $t \geq t_{0}$, all $i \in \mathbb{Z}^{d}$,

$$
\begin{aligned}
\left|\Gamma(i, t)-p_{a t}(i) e^{\alpha_{0} t}\right| & \leq 2 e^{\alpha_{0} t} e^{-(a t)^{1 / 3} / 4}+C t^{-(d+2) / 2} e^{\alpha_{0} t}+C t^{-d / 2-1 / 3} e^{\alpha_{0} t} \\
& \leq C t^{-d / 2-1 / 3} e^{\alpha_{0} t}
\end{aligned}
$$

which is (28).

It remains to deduce (29) from (28). We write

$$
\begin{aligned}
\delta(t, i)= & \left|\int_{0}^{t} \Gamma(i, s) d s-p_{a t}(i) e^{\alpha_{0} t} / \alpha_{0}\right| \\
\leq & \int_{0}^{t-t^{1 / 2}} \Gamma(i, s) d s+\int_{t-t^{1 / 2}}^{t}\left|\Gamma(i, s)-p_{a s}(i) e^{\alpha_{0} s}\right| d s \\
& +\int_{t-t^{1 / 2}}^{t}\left|p_{a s}(i) e^{\alpha_{0} s}-p_{a t}(i) e^{\alpha_{0} s}\right| d s+p_{a t}(i)\left|\int_{t-t^{1 / 2}}^{t} e^{\alpha_{0} s} d s-e^{\alpha_{0} t} / \alpha_{0}\right| \\
= & \delta_{1}(t, i)+\delta_{2}(t, i)+\delta_{3}(t, i)+\delta_{4}(t, i) .
\end{aligned}
$$


First, point (ii) implies that

$$
\delta_{1}(t, i) \leq \int_{0}^{t-t^{1 / 2}} e^{\alpha_{0} s} d s \leq C e^{\alpha_{0}\left(t-t^{1 / 2}\right)} .
$$

Next, (28) tells us, if $t$ is sufficiently large (so that $t-t^{1 / 2} \geq t_{0}$ and $t-t^{1 / 2} \geq t / 2$ ), that

$$
\delta_{2}(t, i) \leq C \int_{t-t^{1 / 2}}^{t} s^{-d / 2-1 / 3} e^{\alpha_{0} s} d s \leq C t^{-d / 2-1 / 3} e^{\alpha_{0} t}
$$

Recalling that $\left|\partial_{t} p_{t}(x)\right| \leq C t^{-d / 2-1}$, we get (still for $t$ large enough so that $t-$ $\left.t^{1 / 2} \geq t / 2\right)$

$$
\delta_{3}(t, i) \leq C \int_{t-t^{1 / 2}}^{t} s^{-d / 2-1}(t-s) e^{\alpha_{0} s} d s \leq C t^{-d / 2-1 / 2} e^{\alpha_{0} t} .
$$

Finally, if $t$ is sufficiently large, we can bound $p_{a t}(i)$ by 1 (for all $i$ ), whence

$$
\delta_{4}(t, i) \leq \alpha_{0}^{-1}\left|e^{\alpha_{0} t}-e^{\alpha_{0}\left(t-t^{1 / 2}\right)}-e^{\alpha_{0} t}\right| \leq C e^{\alpha_{0}\left(t-t^{1 / 2}\right)} .
$$

All in all, we have proved that for all $t$ large enough, all $i \in \mathbb{Z}^{d}$,

$$
\begin{aligned}
& \left|\int_{0}^{t} \Gamma(i, s) d s-p_{a t}(i) e^{\alpha_{0} t} / \alpha_{0}\right| \\
& \quad \leq C e^{\alpha_{0}\left(t-t^{1 / 2}\right)}+C t^{-d / 2-1 / 3} e^{\alpha_{0} t}+C t^{-d / 2-1 / 2} e^{\alpha_{0} t} \leq C t^{-d / 2-1 / 3} e^{\alpha_{0} t}
\end{aligned}
$$

as desired.

We finally can give:

Proof of TheOrem 20. We divide the proof in several steps.

Step 1. As usual, we write

$$
Z_{t}^{i}=\int_{0}^{t} \int_{0}^{\infty} \mathbf{1}_{\left\{z \leq(2 d+1)^{-1} \sum_{j \rightarrow i}\left[\int_{0}^{s-} \varphi(s-u) d Z_{u}^{j}+\varphi(s) \mathbf{1}_{\{j=0\}}\right]\right\}} \pi^{i}(d s d z),
$$

we set $m_{t}^{i}=\mathbb{E}\left[Z_{t}^{i}\right]$ and $\mathbf{m}_{t}=\left(m_{t}^{i}\right)_{i \in \mathbb{Z}^{d}}$. A simple computation, using Lemma 22, shows that

$$
m_{t}^{i}=\int_{0}^{t}(2 d+1)^{-1} \sum_{j \rightarrow i} \varphi(t-s) m_{s}^{j} d s+\mathbf{1}_{\{0 \rightarrow i\}}(2 d+1)^{-1} \int_{0}^{t} \varphi(s) d s .
$$

Using the vector formalism and introducing $\delta=\left(\delta_{i}\right)_{i \in \mathbb{Z}^{d}}$ defined by $\delta_{i}=\mathbf{1}_{\{i=0\}}$, this rewrites $\mathbf{m}_{t}=(A \boldsymbol{\delta}) \int_{0}^{t} \varphi(s) d s+\int_{0}^{t} \varphi(t-s)\left(A \mathbf{m}_{s}\right) d s$. Differentiating this formula (see Lemma 27 for the justification of a very similar differentiation), we find 
$\mathbf{m}_{t}^{\prime}=(A \boldsymbol{\delta}) \varphi(t)+\int_{0}^{t} \varphi(t-s) A \mathbf{m}_{s}^{\prime} d s$, which can be solved as (see Lemma 27 again) $\mathbf{m}_{t}^{\prime}=\sum_{n \geq 1} \varphi^{\star n}(t) A^{n} \boldsymbol{\delta}$. Hence, for all $i \in \mathbb{Z}^{d}$,

$$
\left(m_{t}^{i}\right)^{\prime}=\sum_{n \geq 1} A^{n}(0, i) \varphi^{\star n}(t) .
$$

We introduce the martingales, for $i \in \mathbb{Z}^{d}$ (we use a tilde for compensation),

$$
M_{t}^{i}=\int_{0}^{t} \int_{0}^{\infty} \mathbf{1}_{\left\{z \leq(2 d+1)^{-1} \sum_{j \rightarrow i}\left[\int_{0}^{s-} \varphi(s-u) d Z_{u}^{j}+\varphi(s) \mathbf{1}_{\{j=0\}}\right]\right\}} \tilde{\pi}^{i}(d s d z)
$$

and observe as usual that $\left[M^{i}, M^{j}\right]_{t}=0$ when $i \neq j$ (because these martingales a.s. never jump at the same time) while $\left[M^{i}, M^{i}\right]_{t}=Z_{t}^{i}$. We will use several times that for any family $\left(\alpha_{i}\right)_{i \in \mathbb{Z}^{d}}$,

$$
\mathbb{E}\left[\left(\sum_{i \in \mathbb{Z}^{d}} \alpha_{i} M_{t}^{i}\right)^{2}\right]=\sum_{i \in \mathbb{Z}^{d}} \alpha_{i}^{2} m_{t}^{i}
$$

We finally introduce $U_{t}^{i}=Z_{t}^{i}-m_{t}^{i}$, the vectors $\mathbf{U}_{t}=\left(U_{t}^{i}\right)_{i \in \mathbb{Z}^{d}}$ and $\mathbf{M}_{t}=\left(M_{t}^{i}\right)_{i \in \mathbb{Z}^{d}}$ and observe, exactly as in the proof of Theorem 14, step 1, that $\mathbf{U}_{t}=\mathbf{M}_{t}+$ $A \int_{0}^{t} \varphi(t-s) \mathbf{U}_{s} d s$, whence $\mathbf{U}_{t}=\left(\mathbf{M}_{t}+\sum_{n \geq 1} A^{n} \int_{0}^{t} \varphi^{\star n}(t-s) \mathbf{M}_{s} d s\right)$, and thus, for all $i \in \mathbb{Z}^{d}$,

$$
Z_{t}^{i}=m_{t}^{i}+M_{t}^{i}+\sum_{j \in \mathbb{Z}^{d}} \sum_{n \geq 1} \int_{0}^{t} \varphi^{\star n}(t-s) A^{n}(i, j) M_{s}^{j} d s=m_{t}^{i}+M_{t}^{i}+W_{t}^{i}
$$

the last equality defining $W_{t}^{i}$.

Step 2. Here, we treat the terms $m_{t}^{i}, M_{t}^{i}$, and collect a few more information. Recall that $\Gamma(t, i)=\sum_{n \geq 1} A^{n}(0, i) \varphi^{\star n}(t)$. Starting from (31), we see that $m_{t}^{i}=$ $\int_{0}^{t} \Gamma(i, s) d s$ and deduce from Lemma 21(iv) that there are $C$ and $t_{0} \geq 0$ such that for all $i \in \mathbb{Z}^{d}$, all $t \geq t_{0}$,

$$
\left|m_{t}^{i}-\frac{1}{\alpha_{0}} p_{a t}(i) e^{\alpha_{0} t}\right| \leq \frac{C e^{\alpha_{0} t}}{t^{d / 2+1 / 3}} .
$$

Next, Lemma 21(ii)-(iii) imply that $\sum_{i \in \mathbb{Z}^{d}} m_{t}^{i}=\int_{0}^{t} e^{\alpha_{0} s} d s \leq C e^{\alpha_{0} t}$ and $\sum_{i \in \mathbb{Z}^{d}}|i|^{2} m_{t}^{i} \leq C \int_{0}^{t}(1+s) e^{\alpha_{0} s} d s \leq C(1+t) e^{\alpha_{0} t}$. Finally, we observe that

$$
\mathbb{E}\left[\left|M_{t}^{i}\right|\right] \leq\left(m_{t}^{i}\right)^{1 / 2} \leq\left(\sum_{j \in \mathbb{Z}^{d}} m_{t}^{j}\right)^{1 / 2} \leq C e^{\alpha_{0} t / 2}
$$

Step 3. We introduce

$$
X=\sum_{j \in \mathbb{Z}^{d}} \int_{0}^{\infty} e^{-\alpha_{0} s} M_{s}^{j} d s
$$


and show that there are $C>0$ and $t_{0}>0$ such that for all $i \in \mathbb{Z}^{d}$, all $t \geq t_{0}$,

$$
\mathbb{E}\left[\left|W_{t}^{i}-p_{a t}(i) e^{\alpha_{0} t} X\right|\right] \leq \frac{C e^{\alpha_{0} t}}{t^{d / 2+1 / 3}} .
$$

We observe that since $A^{n}(i, j)=A^{n}(0, i-j)$, it holds that $W_{t}^{i}=\sum_{j \in \mathbb{Z}^{d}} \int_{0}^{t} \Gamma(i-$ $j, t-s) M_{S}^{j} d s$. We also introduce the auxiliary processes

$$
\begin{aligned}
& \bar{W}_{t}^{i}=\sum_{j \in \mathbb{Z}^{d}} \int_{0}^{t^{1 / 2}} \Gamma(i-j, t-s) M_{s}^{j} d s, \\
& \widetilde{W}_{t}^{i}=\sum_{j \in \mathbb{Z}^{d}} \int_{0}^{t^{1 / 2}} p_{a(t-s)}(i-j) e^{\alpha_{0}(t-s)} M_{s}^{j} d s, \\
& \widehat{W}_{t}^{i}=\sum_{j \in \mathbb{Z}^{d}} \int_{0}^{t^{1 / 2}} p_{a t}(i) e^{\alpha_{0}(t-s)} M_{s}^{j} d s .
\end{aligned}
$$

Step 3.1. Here, we show that $\mathbb{E}\left[\left|W_{t}^{i}-\bar{W}_{t}^{i}\right|\right] \leq C \exp \left(\alpha_{0} t-\left(\alpha_{0} / 2\right) t^{1 / 2}\right)$. By definition of $\Gamma$ and using (32),

$$
\begin{aligned}
\mathbb{E}\left[\left|W_{t}^{i}-\bar{W}_{t}^{i}\right|\right] & \leq \int_{t^{1 / 2}}^{t} \sum_{n \geq 1} \varphi^{\star n}(t-s) \mathbb{E}\left[\left|\sum_{j \in \mathbb{Z}^{d}} A^{n}(i, j) M_{s}^{j}\right|\right] d s \\
& \leq \int_{t^{1 / 2}}^{t} \sum_{n \geq 1} \varphi^{\star n}(t-s)\left(\sum_{j \in \mathbb{Z}^{d}}\left(A^{n}(i, j)\right)^{2} m_{s}^{j}\right)^{1 / 2} d s .
\end{aligned}
$$

Using that $A^{n}(i, j)$ is bounded by 1 and that $\sum_{i \in \mathbb{Z}^{d}} m_{t}^{i} \leq C e^{\alpha_{0} t}$ (see step 2), we see that $\sum_{j \in \mathbb{Z}^{d}}\left(A^{n}(i, j)\right)^{2} m_{s}^{j} \leq C e^{\alpha_{0} s / 2}$. Next, the explicit expression of $\varphi^{\star n}$ [see Lemma 21(i)] gives $\sum_{n \geq 1} \varphi^{\star n}(t-s)=e^{\alpha_{0}(t-s)}$. We finally find

$$
\mathbb{E}\left[\left|W_{t}^{i}-\bar{W}_{t}^{i}\right|\right] \leq C \int_{t^{1 / 2}}^{t} e^{\alpha_{0}(t-s)} e^{\alpha_{0} s / 2} d s \leq C e^{\alpha_{0} t} e^{-\alpha_{0} t^{1 / 2} / 2} .
$$

Step 3.2. We next check that $\mathbb{E}\left[\left|\bar{W}_{t}^{i}-\widetilde{W}_{t}^{i}\right|\right] \leq C t^{-d / 2-1 / 3} e^{\alpha_{0} t}$. Using (32), we get

$\mathbb{E}\left[\left|\bar{W}_{t}^{i}-\widetilde{W}_{t}^{i}\right|\right] \leq \int_{0}^{t^{1 / 2}}\left[\sum_{j \in \mathbb{Z}^{d}} m_{s}^{j}\left(\Gamma(i-j, t-s)-p_{a(t-s)}(i-j) e^{\alpha_{0}(t-s)}\right)^{2}\right]^{1 / 2} d s$.

Using Lemma 21(iv), $\left|\Gamma(i-j, t-s)-p_{a(t-s)}(i-j) e^{\alpha_{0}(t-s)}\right| \leq C(t-$ $s)^{-d / 2-1 / 3} e^{\alpha_{0}(t-s)}$ if $t-s$ is large enough (which is the case for all $s \in\left[0, t^{1 / 2}\right]$ if $t$ is large enough). Since furthermore $\sum_{i \in \mathbb{Z}^{d}} m_{t}^{i} \leq C e^{\alpha_{0} t}$ (see step 2), we find

$$
\mathbb{E}\left[\left|\bar{W}_{t}^{i}-\widetilde{W}_{t}^{i}\right|\right] \leq C e^{\alpha_{0} t} \int_{0}^{t^{1 / 2}}(t-s)^{-d / 2-1 / 3} e^{-\alpha_{0} s / 2} d s
$$


For $t$ large enough, we clearly have $(t-s)^{-d / 2-1 / 3} \leq 2 t^{-d / 2-1 / 3}$ for all $s \in$ $\left[0, t^{1 / 2}\right]$, whence

$$
\mathbb{E}\left[\left|\bar{W}_{t}^{i}-\widetilde{W}_{t}^{i}\right|\right] \leq C t^{-d / 2-1 / 3} e^{\alpha_{0} t} \int_{0}^{t^{1 / 2}} e^{-\alpha_{0} s / 2} d s \leq C t^{-d / 2-1 / 3} e^{\alpha_{0} t} .
$$

Step 3.3. We now prove that $\mathbb{E}\left[\left|\widetilde{W}_{t}^{i}-\widehat{W}_{t}^{i}\right|\right] \leq C t^{-d / 2-1 / 2} e^{\alpha_{0} t}$. As usual, we start with

$$
\mathbb{E}\left[\left|\widetilde{W}_{t}^{i}-\widehat{W}_{t}^{i}\right|\right] \leq \int_{0}^{t^{1 / 2}}\left(\sum_{j \in \mathbb{Z}^{d}} m_{s}^{j}\left(p_{a(t-s)}(i-j)-p_{a t}(i)\right)^{2}\right)^{1 / 2} e^{\alpha_{0}(t-s)} d s .
$$

But an easy computation (using that $\left|\partial_{t} p_{t}(x)\right| \leq C t^{-d / 2-1}$ and $\left|\nabla_{x} p_{t}(x)\right| \leq$ $\left.C t^{-d / 2-1 / 2}\right)$ shows that for all $t>0$, all $h \in(0, t / 2)$, all $x, y \in \mathbb{R}^{d}, \mid p_{t-h}(x-$ $y)-p_{t}(x)\left|\leq C h t^{-d / 2-1}+\right| y \mid t^{-d / 2-1 / 2}$. Hence, if $t$ is large enough so that $t-t^{1 / 2} \geq t / 2$, we can write

$$
\begin{aligned}
\mathbb{E}\left[\left|\widetilde{W}_{t}^{i}-\widehat{W}_{t}^{i}\right|\right] \leq & C \int_{0}^{t^{1 / 2}}\left(\sum_{j \in \mathbb{Z}^{d}} m_{s}^{j}\left(s t^{-d / 2-1}+|j| t^{-d / 2-1 / 2}\right)^{2}\right)^{1 / 2} e^{\alpha_{0}(t-s)} d s \\
\leq & C t^{-d / 2-1 / 2} \int_{0}^{t^{1 / 2}}\left(\sum_{j \in \mathbb{Z}^{d}} m_{s}^{j}\right)^{1 / 2} e^{\alpha_{0}(t-s)} d s \\
& +C t^{-d / 2-1 / 2} \int_{0}^{t^{1 / 2}}\left(\sum_{j \in \mathbb{Z}^{d}}|j|^{2} m_{s}^{j}\right)^{1 / 2} e^{\alpha_{0}(t-s)} d s .
\end{aligned}
$$

Finally, we know from step 2 that $\sum_{j \in \mathbb{Z}^{d}} m_{s}^{j}+\sum_{j \in \mathbb{Z}^{d}}|j|^{2} m_{s}^{j} \leq C(1+s) e^{\alpha_{0} s}$, whence

$$
\mathbb{E}\left[\left|\widetilde{W}_{t}^{i}-\widehat{W}_{t}^{i}\right|\right] \leq C t^{-d / 2-1 / 2} \int_{0}^{t^{1 / 2}}(1+s)^{1 / 2} e^{\alpha_{0} s / 2} e^{\alpha_{0}(t-s)} d s \leq C t^{-d / 2-1 / 2} e^{\alpha_{0} t}
$$

Step 3.4. We finally verify that $\mathbb{E}\left[\left|\widehat{W}_{t}^{i}-p_{a t}(i) e^{\alpha_{0} t} X\right|\right] \leq C e^{\alpha_{0} t-\left(\alpha_{0} / 2\right) t^{1 / 2}}$. We note that

$$
\widehat{W}_{t}^{i}-p_{a t}(i) e^{\alpha_{0} t} X=p_{a t}(i) e^{\alpha_{0} t} \sum_{j \in \mathbb{Z}^{d}} \int_{t^{1 / 2}}^{t} e^{-\alpha_{0} s} M_{s}^{j} d s .
$$

For $t$ large enough (not depending on $i$ ), we can bound $p_{a t}(i)$ by 1 . Hence, we infer from (32) and the fact that $\sum_{j \in \mathbb{Z}^{d}} m_{s}^{j} \leq C e^{\alpha_{0} s}$ that

$$
\begin{aligned}
\mathbb{E}\left[\left|\widehat{W}_{t}^{i}-p_{a t}(i) e^{\alpha_{0} t} X\right|\right] & \leq C e^{\alpha_{0} t} \int_{t^{1 / 2}}^{t} e^{-\alpha_{0} s}\left(\sum_{j \in \mathbb{Z}^{d}} m_{s}^{j}\right)^{1 / 2} d s \\
& \leq C e^{\alpha_{0} t} \int_{t^{1 / 2}}^{\infty} e^{-\alpha_{0} s / 2} d s,
\end{aligned}
$$

from which the conclusion follows. 
Step 3.5. Gathering steps 3.1 to 3.4 , we conclude that indeed, there are $C>$ 0 and $t_{0}>0$ such that for all $t \geq t_{0}$, all $i \in \mathbb{Z}^{d}, \mathbb{E}\left[\left|W_{t}^{i}-p_{a t}(i) e^{\alpha_{0} t} X\right|\right] \leq$ $C e^{\alpha_{0} t} t^{-d / 2-1 / 3}$.

Step 4. Define the random variable $H=\alpha_{0}^{-1}+X$. Recall (33) and write $Z_{t}^{i}-$ $p_{a t}(i) e^{\alpha_{0} t} H=\left[m_{t}^{i}-\alpha_{0}^{-1} p_{a t}(i) e^{\alpha_{0} t}\right]+M_{t}^{i}+\left[W_{t}^{i}-p_{a t}(i) e^{\alpha_{0} t} X\right]$. Gathering steps 2 and 3 , we see that there are $C>0$ and $t_{0}>0$ such that for all $t \geq t_{0}$, all $i \in \mathbb{Z}^{d}$,

$$
\mathbb{E}\left[\left|Z_{t}^{i}-p_{a t}(i) e^{\alpha_{0} t} H\right|\right] \leq \frac{C e^{\alpha_{0} t}}{t^{d / 2+1 / 3}}+C e^{\alpha_{0} t / 2} \leq \frac{C e^{\alpha_{0} t}}{t^{d / 2+1 / 3}} .
$$

To prove that $H$ is nonnegative, it suffices to use the above inequality with $i=0$, divided by $p_{a t}(0) e^{\alpha_{0} t}$. Recalling that $p_{a t}(0)=c t^{-d / 2}$ for some constant $c$, we deduce that $\mathbb{E}\left[\left|H-Z_{t}^{0} e^{-\alpha_{0} t} / p_{a t}(0)\right|\right] \leq C t^{-1 / 3}$. Consequently, $H$ is the limit (in $\left.L^{1}\right)$ of $Z_{t}^{0} e^{-\alpha_{0} t} / p_{a t}(0)$, and is thus nonnegative. This completes the proof of (i).

Step 5. We now check (ii), which follows from (i): for $x \in \mathbb{R}^{d}$, and $t \geq t_{0}$,

$$
\begin{gathered}
\mathbb{E}\left[\left|t^{d / 2} e^{-\alpha_{0} t} Z_{t}^{\left\lfloor x t^{1 / 2}\right\rfloor}-H p_{a}(x)\right|\right] \leq \\
t^{d / 2} e^{-\alpha_{0} t} \mathbb{E}\left[\left|Z_{t}^{\left\lfloor x t^{1 / 2}\right\rfloor}-H e^{\alpha_{0} t} p_{a t}\left(\left\lfloor x t^{1 / 2}\right\rfloor\right)\right|\right] \\
+\mathbb{E}[H]\left|t^{d / 2} p_{a t}\left(\left\lfloor x t^{1 / 2}\right\rfloor\right)-p_{a}(x)\right| .
\end{gathered}
$$

The first term on the RHS is bounded, by (i), by $C t^{-1 / 3}$, which tends to 0 as $t \rightarrow \infty$. The second term also tends to 0 , simply because

$$
t^{d / 2} p_{a t}\left(\left\lfloor x t^{1 / 2}\right\rfloor\right)=p_{a}\left(t^{-1 / 2}\left\lfloor x t^{1 / 2}\right\rfloor\right) \rightarrow p_{a}(x) \quad \text { as } t \rightarrow \infty .
$$

Step 6. It remains to prove (iii) and (iv). The fact that $H=\lim _{t \rightarrow \infty}(4 \pi t /$ $(2 d+1))^{d / 2} e^{-\alpha_{0} t} Z_{t}^{0}$ in $L^{1}$ follows from point (i) with $i=0$. Next, note that $H=\lim _{t \rightarrow \infty} e^{-\alpha_{0} t} Z_{t}$ in $L^{1}$, where $Z_{t}=\sum_{i \in \mathbb{Z}^{d}} Z_{t}^{i}$. Indeed, recalling (33)

$$
Z_{t}=\sum_{i \in \mathbb{Z}^{d}} m_{t}^{i}+\sum_{i \in \mathbb{Z}^{d}} M_{t}^{i}+\sum_{i \in \mathbb{Z}^{d}} W_{t}^{i}
$$

We have $\sum_{i \in \mathbb{Z}^{d}} m_{t}^{i}=\int_{0}^{t} e^{\alpha_{0} s} d s=\alpha_{0}^{-1}\left[e^{\alpha_{0} t}-1\right]$ by step 2, (32) implies that $\mathbb{E}\left[\left(\sum_{i \in \mathbb{Z}^{d}} M_{t}^{i}\right)^{2}\right]=\sum_{i \in \mathbb{Z}^{d}} m_{t}^{i}$ and finally $\sum_{i \in \mathbb{Z}^{d}} W_{t}^{i}=e^{\alpha_{0} t} \int_{0}^{t} e^{-\alpha_{0} s} \sum_{i \in \mathbb{Z}^{d}} M_{s}^{i} d s$, therefore, $e^{-\alpha_{0} t} Z_{t}$ converges to $\alpha_{0}^{-1}+0+X=H$ as $t \rightarrow \infty$ in $L^{1}$. We also note that $\mathbb{E}[H]=\alpha_{0}^{-1}>0$ since $\mathbb{E}[X]=0$.

Next, we recall that by Theorem 19(i), $\left(Z_{t}\right)_{t \geq 0}$ is a scalar impulsion Hawkes process: its compensator is given by $A_{t}=\int_{0}^{t}\left[\varphi(s)+\int_{0}^{s-} \varphi(s-u) d Z_{u}\right] d s$. We claim that $\left(Z_{t}\right)_{t \geq 0}$ has the same law as $\left(\widetilde{Z}_{t}\right)_{t \geq 0}$ built as follows:

- consider a Poisson process $\left(N_{t}\right)_{t \geq 0}$ with intensity $\varphi(t) d t$, observe that $N_{\infty}$ is Poisson $(\Lambda)$-distributed, denote by $0<T_{1}<\cdots<T_{N_{\infty}}$ its times of jump (we adopt the convention that $T_{i}=\infty$ for $i>N_{\infty}$ ),

- consider an i.i.d. family $\left(\widetilde{Z}_{t}^{k}\right)_{t \geq 0}$ of scalar impulsion Hawkes process with same law as $\left(Z_{t}\right)_{t \geq 0}$,

- put $\widetilde{Z}_{t}=N_{t}+\sum_{i=1}^{N_{\infty}} \widetilde{Z}_{t-T_{k}}^{k} \mathbf{1}_{\left\{t \geq T_{k}\right\}}$. 
Indeed, $\left(\widetilde{Z}_{t}\right)_{t \geq 0}$ is a counting process with compensator

$$
\begin{gathered}
\int_{0}^{t}\left[\varphi(s)+\sum_{i \geq 1} 1_{\left\{s>T_{k}\right\}}\left(\varphi\left(s-T_{k}\right)+\int_{0}^{\left(s-T_{k}\right)-} \varphi\left(s-T_{k}-u\right) d \widetilde{Z}_{u}^{k}\right)\right] d s \\
=\int_{0}^{t}\left[\varphi(s)+\int_{0}^{s-} \varphi(s-u) d \widetilde{Z}_{u}\right] d s .
\end{gathered}
$$

We define $\tilde{H}=\lim _{t \rightarrow \infty} e^{-\alpha_{0} t} \widetilde{Z}_{t}$ and, for each $k \geq 1, \widetilde{H}_{k}=\lim _{t \rightarrow \infty} e^{-\alpha_{0} t} \widetilde{Z}_{t}^{k}$. We obviously have $\widetilde{H}=\sum_{k=1}^{N_{\infty}} e^{-\alpha_{0} T_{k}} \widetilde{H}_{k}$. Denoting by $p=\operatorname{Pr}(H=0)$ [which also equals $\operatorname{Pr}(\tilde{H}=0)$ and $\operatorname{Pr}\left(\widetilde{H}_{k}=0\right)$ for all $\left.k \geq 1\right]$, we deduce by independence of the family $\left(\widetilde{H}_{k}\right)_{k \geq 1}$, that $p=\sum_{n \geq 1} \operatorname{Pr}\left(N_{\infty}=n\right) p^{n}$. Hence $p=\sum_{n \geq 1} e^{-\Lambda} \Lambda^{n} p^{n} / n !=$ $e^{-\Lambda(1-p)}$. Since $\mathbb{E}[H]>0$, we cannot have $p=1$. Hence $p$ is the unique solution in $(0,1)$ to $p=e^{-\Lambda(1-p)}$. Recalling that $\operatorname{Pr}\left(\Omega_{e}\right)=\exp \left(-\gamma_{\Lambda} \Lambda\right)$ where $\gamma_{\Lambda} \in(0,1)$ is characterised by $\gamma_{\Lambda} \Lambda+\log \left(1-\gamma_{\Lambda}\right)=0$ by Theorem 19 , 2(ii), we easily check that $p=\operatorname{Pr}\left(\Omega_{e}\right)$.

By definition of $H$, we have $\Omega_{e} \subset\{H=0\}$. Since $\operatorname{Pr}\left(\Omega_{e}\right)=\operatorname{Pr}(H=0)$ we conclude that a.s., $H>0$ on $\Omega_{e}^{c}$.

\section{APPENDIX: CONVOLUTION EQUATIONS}

We collect here some technical results about convolution equations. We start with an identity of constant use in the paper.

LEMMA 22. Let $\phi:[0, \infty) \mapsto \mathbb{R}$ be locally integrable and let $\alpha:[0, \infty) \mapsto \mathbb{R}$ have finite variations on compact intervals and satisfy $\alpha(0)=0$. Then for all $t \geq 0$,

$$
\int_{0}^{t} \int_{0}^{s-} \phi(s-u) d \alpha(u) d s=\int_{0}^{t} \int_{0}^{s} \phi(s-u) d \alpha(u) d s=\int_{0}^{t} \phi(t-s) \alpha(s) d s .
$$

Proof. First, we clearly have that $\int_{0}^{s-} \phi(s-u) d \alpha(u)=\int_{0}^{s} \phi(s-u) d \alpha(u)$ for almost every $s \geq 0$, whence $\int_{0}^{t} \int_{0}^{s-} \phi(s-u) d \alpha(u) d s=\int_{0}^{t} \int_{0}^{s} \phi(s-u) d \alpha(u) d s$. Using twice the Fubini theorem,

$$
\begin{aligned}
\int_{0}^{t}\left(\int_{0}^{s} \phi(s-u) d \alpha(u)\right) d s & =\int_{0}^{t}\left(\int_{u}^{t} \phi(s-u) d s\right) d \alpha(u) \\
& =\int_{0}^{t}\left(\int_{0}^{t-u} \phi(v) d v\right) d \alpha(u) \\
& =\int_{0}^{t}\left(\int_{0}^{t-v} d \alpha(u)\right) \phi(v) d v \\
& =\int_{0}^{t} \alpha(t-v) \phi(v) d v
\end{aligned}
$$

from which the conclusion follows, using the substitution $s=t-v$. 
We carry on with a generalised Grönwall-Picard lemma, which is more or less standard.

LEMMA 23. Let $\phi:[0, \infty) \mapsto[0, \infty)$ be locally integrable and $g:[0, \infty) \mapsto$ $[0, \infty)$ be locally bounded.

(i) Consider a locally bounded nonnegative function $u$ such that for all $t \geq 0$, $u_{t} \leq g_{t}+\int_{0}^{t} \phi(t-s) u_{s} d s$ for all $t \geq 0$. Then $\sup _{[0, T]} u_{t} \leq C_{T} \sup _{[0, T]} g_{t}$, for some constant $C_{T}$ depending only on $T>0$ and $\phi$.

(ii) Consider a sequence of locally bounded nonnegative functions $u^{n}$ such that for all $t \geq 0$, all $n \geq 0, u_{t}^{n+1} \leq \int_{0}^{t} \phi(t-s) u_{s}^{n} d s$. Then $\sup _{[0, T]} \sum_{n \geq 0} u_{t}^{n} \leq C_{T}$, for some constant $C_{T}$ depending only on $T>0, u^{0}$ and $\phi$.

(iii) Consider a sequence of locally bounded nonnegative functions $u^{n}$ such that for all $t \geq 0$, all $n \geq 0, u_{t}^{n+1} \leq g_{t}+\int_{0}^{t} \phi(t-s) u_{s}^{n} d s$. Then for all $T \geq 0$, $\sup _{[0, T]} \sup _{n \geq 0} u_{t}^{n} \leq C_{T}$, for some constant $C_{T}$ depending only on $T>0, u^{0}, g$ and $\phi$.

ProOF. We start with point (i). Fix $T>0$ and consider $A>0$ such that $\int_{0}^{T} \phi(s) \mathbf{1}_{\{\phi(s) \geq A\}} d s \leq 1 / 2$. Then for all $t \in[0, T]$,

$$
\begin{aligned}
u_{t} & \leq g_{t}+\int_{0}^{t} \mathbf{1}_{\{\phi(t-s) \leq A\}} \phi(t-s) u_{s} d s+\int_{0}^{t} \mathbf{1}_{\{\phi(t-s)>A\}} \phi(t-s) u_{s} d s \\
& \leq g_{t}+A \int_{0}^{t} u_{s} d s+\sup _{[0, t]} u_{s} / 2,
\end{aligned}
$$

from which we deduce that $\sup _{[0, t]} u_{s} \leq 2 \sup _{[0, t]} g_{s}+2 A \int_{0}^{t} u_{s} d s$. We then can apply the standard Grönwall lemma to get $\sup _{[0, T]} u_{s} \leq 2\left(\sup _{[0, T]} g_{S}\right) e^{2 A T}$.

To check point (iii), put $v_{t}^{n}=\sup _{k=0, \ldots, n} u_{t}^{k}$. One easily checks that for all $n \geq 0$, $v_{t}^{n} \leq u_{t}^{0}+g_{t}+\int_{0}^{t} \phi(t-s) v_{s}^{n} d s$. By point (i), $\sup _{[0, T]} v_{t}^{n} \leq C_{T} \sup _{[0, T]}\left(g_{t}+u_{t}^{0}\right)$. Letting $n$ increase to infinity completes the proof.

Point (ii) follows from point (iii), since $v_{t}^{n}=\sum_{k=0}^{n} u_{t}^{k}$ satisfies $v_{t}^{n+1} \leq u_{t}^{0}+$ $\int_{0}^{t} \phi(t-s) v_{s}^{n} d s$.

We next prove an easy well-posedness result for a general convolution equation.

LEMMA 24. Let $h: \mathbb{R} \mapsto[0, \infty)$ be Lipschitz-continuous and $\varphi:[0, \infty) \mapsto \mathbb{R}$ be locally integrable. The equation

$$
m_{t}=\int_{0}^{t} h\left(\int_{0}^{s} \varphi(s-u) d m_{u}\right) d s
$$

has a unique nondecreasing locally bounded solution. Furthermore, $m$ is of class $C^{1}$ on $[0, \infty)$. 
If $h(x)=\mu+x$ for some $\mu>0$ and if $\varphi$ is nonnegative, equation (34) rewrites as

$$
m_{t}=\mu t+\int_{0}^{t} \varphi(t-s) m_{s} d s .
$$

PROOF. Let $m$ and $\tilde{m}$ be two such solutions. Since $h$ is Lipschitz-continuous,

$$
\begin{aligned}
v_{t} & =\int_{0}^{t}\left|d\left(m_{u}-\tilde{m}_{u}\right)\right| \leq C \int_{0}^{t} d s \int_{0}^{s}|\varphi(s-u)|\left|d\left(m_{u}-\tilde{m}_{u}\right)\right| \\
& =C \int_{0}^{t}|\varphi(t-u)| v_{u} d u .
\end{aligned}
$$

The last inequality follows from Lemma 22. Lemma 23(i) allows us to conclude that $v_{t}=0$ for all $t \geq 0$ (because $v_{t} \leq m_{t}+\tilde{m}_{t}$ is locally bounded), whence $m_{t}=\tilde{m}_{t}$ for all $t \geq 0$. For the existence, we consider the sequence of nondecreasing functions $m_{t}^{0}=0$ and $m_{t}^{n+1}=\int_{0}^{t} h\left(\int_{0}^{s} \varphi(s-u) d m_{u}^{n}\right) d s$ for every $n \geq 0$. We easily check that $m_{t}^{n+1} \leq h(0) t+|h|_{\operatorname{lip}} \int_{0}^{t}|\varphi(t-u)| m_{u}^{n} d u$, so that $\sup _{n \geq 0} m_{t}^{n}$ is locally bounded by Lemma 23(iii). Setting, for $n \geq 0, \delta_{t}^{n}=\int_{0}^{t}\left|d\left(m_{u}^{n+1}-m_{u}^{n}\right)\right|$, one readily gets $\delta_{t}^{n+1} \leq|h|_{\operatorname{lip}} \int_{0}^{t}|\varphi(s-u)| \delta_{u}^{n} d u$ for all $n \geq 0$. Lemma 23(ii) thus implies that $\sum_{n \geq 0} \delta_{t}^{n}<\infty$. All this classically implies the existence of a locally bounded nondecreasing $m$ such that for all $t \geq 0, \lim _{n} \int_{0}^{t}\left|d\left(m_{u}-m_{u}^{n}\right)\right|=0$. Checking that $m$ solves (34) is routine.

To prove that $m$ is $C^{1}$, we use the previous Picard iteration. One easily sees, by induction, that $m^{n}$ is $C^{1}$ for all $n \geq 0$ and that $\left(m_{t}^{n+1}\right)^{\prime}=h\left(\int_{0}^{t} \varphi(t-u)\left(m_{u}^{n}\right)^{\prime} d u\right)$ [indeed, $t \mapsto \int_{0}^{t} \varphi(t-u)\left(m_{u}^{n}\right)^{\prime} d u=\int_{0}^{t} \varphi(u)\left(m_{t-u}^{n}\right)^{\prime} d u$ is continuous because $\varphi$ is locally integrable and because $\left(m_{t}^{n}\right)^{\prime}$ is continuous by the inductive assumption]. Next, a direct computation shows that $\left|\left(m_{t}^{n+1}\right)^{\prime}-\left(m_{t}^{n}\right)^{\prime}\right| \leq C \int_{0}^{t}|\varphi(t-u)| \mid\left(m_{u}^{n}\right)^{\prime}-$ $\left(m_{u}^{n-1}\right)^{\prime} \mid d u$. Using Lemma 23(ii), we deduce that the sequence $\left(m^{n}\right)^{\prime}$ is Cauchy (for the uniform convergence on compact time intervals). The conclusion classically follows.

The equivalence between (34) and (35) in the linear case directly follows from Lemma 22.

We now investigate the large-time behaviour of $m_{t}$ in the linear case. We start with the subcritical case.

LEMMA 25. Consider $\mu>0$ and a function $\varphi:(0, \infty) \mapsto[0, \infty)$ such that $\Lambda=\int_{0}^{\infty} \varphi(s) d s<1$. By Lemma 24, (35) has a unique nondecreasing locally bounded solution $\left(m_{t}\right)_{t \geq 0}$, which is furthermore of class $C^{1}$. It holds that $m_{t}^{\prime} \sim a_{0}$ and $m_{t} \sim a_{0} t$ as $t \rightarrow \infty$, where $a_{0}=\mu /(1-\Lambda)>0$.

ProOF. We rather use (34), which writes $m_{t}=\mu t+\int_{0}^{t} \int_{0}^{s} \varphi(s-u) m_{u}^{\prime} d u d s$. Differentiating this expression, we find $m_{t}^{\prime}=\mu+\int_{0}^{t} \varphi(t-s) m_{s}^{\prime} d s$. We first 
introduce $u_{t}=\sup _{[0, t]} m_{s}^{\prime}$. We have $u_{t} \leq \mu+\Lambda u_{t}$, whence $u_{t} \leq \mu /(1-\Lambda)$ for all $t \geq 0$ and thus $\limsup _{t \rightarrow \infty} m_{t}^{\prime} \leq \mu /(1-\Lambda)$. We next introduce $v_{t}=$ $\inf _{s \geq t} m_{s}^{\prime}$, which is nondecreasing, and thus has a limit $\ell \in(0, \infty]$. We have $v_{t} \geq \mu+v_{t / 2} \int_{0}^{t / 2} \varphi(s) d s \rightarrow \mu+\Lambda \ell$ as $t \rightarrow \infty$. Consequently $\ell \geq \mu+\Lambda \ell$, whence $\ell \geq \mu /(1-\Lambda)$ and finally $\liminf _{t \rightarrow \infty} m_{t}^{\prime} \geq \mu /(1-\Lambda)$. All this proves that $m_{t}^{\prime} \sim a_{0}$ and this implies that $m_{t} \sim a_{0} t$.

We now turn to the supercritical case.

LEMMA 26. Consider $\mu>0$ and a function $\varphi:[0, \infty) \mapsto[0, \infty)$ such that $\Lambda=\int_{0}^{\infty} \varphi(s) d s \in(1, \infty]$. By Lemma 24 , (35) has a unique nondecreasing locally bounded solution $\left(m_{t}\right)_{t \geq 0}$, which is of class $C^{1}$. Assume furthermore that $t \mapsto$ $\int_{0}^{t}|d \varphi(s)|$ has at most polynomial growth. Let $\Gamma(t)=\sum_{n \geq 1} \varphi^{\star n}(t)$ and $\Upsilon(t)=$ $\int_{0}^{t} \Gamma(s) d s$.

(a) There is a unique $\alpha_{0}>0$ such that $\mathcal{L}_{\varphi}\left(\alpha_{0}\right)=1$. The function $\Gamma$ is locally bounded. Setting $a_{0}=\mu \alpha_{0}^{-2}\left(\int_{0}^{\infty} t \varphi(t) e^{-\alpha_{0} t} d t\right)^{-1}$, we have, as $t \rightarrow \infty$,

$$
\begin{aligned}
\Gamma(t) & \sim\left(a_{0} \alpha_{0}^{2} / \mu\right) e^{\alpha_{0} t}, \quad \Upsilon(t) \sim\left(a_{0} \alpha_{0} / \mu\right) e^{\alpha_{0} t}, \\
m_{t} & \sim a_{0} e^{\alpha_{0} t}, \quad m_{t}^{\prime} \sim a_{0} \alpha_{0} e^{\alpha_{0} t} .
\end{aligned}
$$

(b) There are some constants $0<c<C$ such that for all $t \geq 0, c e^{\alpha_{0} t} \leq \Gamma(t)+$ $1 \leq C e^{\alpha_{0} t}, c e^{\alpha_{0} t} \leq \Upsilon(t)+1 \leq C e^{\alpha_{0} t}, c e^{\alpha_{0} t} \leq m_{t}+1 \leq C e^{\alpha_{0} t}$ and $c e^{\alpha_{0} t} \leq m_{t}^{\prime}+$ $1 \leq C e^{\alpha_{0} t}$

(c) We also have

$$
\lim _{t \rightarrow \infty} \int_{0}^{t}\left(\frac{\Upsilon(t-s)}{m_{t}}-\frac{\alpha_{0}}{\mu} e^{-\alpha_{0} s}\right)^{2} m_{s}^{\prime} d s=0 .
$$

(d) Consider a real sequence $\left(\eta_{n}\right)_{n \geq 1}$ such that $\lim _{n \rightarrow \infty} \eta_{n}=0$. Then we have the property that $\lim _{t \rightarrow \infty} e^{-\alpha_{0} t} \sum_{n \geq 1} \eta_{n} \int_{0}^{t} \varphi^{\star n}(t-s) e^{\alpha_{0} s / 2} d s=0$.

(e) For any pair of locally bounded functions $u, h:(0, \infty) \mapsto \mathbb{R}$ such that $u=$ $h+u \star \varphi$, there holds $u=h+h \star \Gamma$.

PROOF. We easily deduce from our assumptions on $\varphi$ that there is some constants $C>0, p>0$ such that for all $t \geq 0, \varphi(t) \leq C(1+t)^{p}$ (in particular, $\varphi$ is locally bounded). Hence, its Laplace transform is clearly well defined on $(0, \infty)$, of class $C^{\infty}$, and $\lim _{\alpha \rightarrow \infty} \mathcal{L}_{\varphi}(\alpha)=0$. Furthermore, $\mathcal{L}_{\varphi}(0)=\int_{0}^{\infty} \varphi(t) d t \in(1, \infty]$. Hence, there indeed exists a unique $\alpha_{0}>0$ such that $\mathcal{L}_{\varphi}\left(\alpha_{0}\right)=1$. We now divide the proof into several steps.

Step 1. We first prove that $\Gamma$ is locally bounded. To this end, we introduce $\Gamma_{n}=$ $\sum_{k=1}^{n} \varphi^{\star k}(t)$ and observe that $\Gamma_{n+1}=\varphi+\Gamma_{n} \star \varphi$. Since $\varphi$ is locally bounded, Lemma 23(iii) allows us to conclude that $\sup _{n} \Gamma_{n}$ is locally bounded, whence the result. 
Step 2 . Here, we prove (e). Since $h$ is locally bounded and since $\varphi$ is locally integrable, we easily deduce from Lemma 23(i) that the equation $v=h+v \star \varphi$ (with unknown $v$ ) has at most one locally bounded solution. Since both $u$ and $h+h \star \Gamma$ are locally bounded solutions, the conclusion follows.

Step 3. The aim of this step is to verify that $\Gamma(t) \sim\left(a_{0} \alpha_{0}^{2} / \mu\right) e^{\alpha_{0} t}$ as $t \rightarrow \infty$. Observe that $\Gamma$ solves $\Gamma=\varphi+\Gamma \star \varphi$ and introduce $u(t)=\Gamma(t) e^{-\alpha_{0} t}$ and $f(t)=$ $\varphi(t) e^{-\alpha_{0} t}$. One easily checks that $u=f+u \star f$. We now apply Theorem 4 of Feller [13]. We have $\int_{0}^{\infty} f(t) d t=1$ by definition of $\alpha_{0}$. We set $b_{1}=\int_{0}^{\infty} t f(t) d t=$ $\int_{0}^{\infty} t \varphi(t) e^{-\alpha_{0} t} d t$ and $b_{2}=\int_{0}^{\infty} t^{2} f(t) d t=\int_{0}^{\infty} t^{2} \varphi(t) e^{-\alpha_{0} t} d t$, which clearly both converge, since $\varphi(t) \leq C(1+t)^{p}$. Finally, it is not difficult to check that $f(t)$, $t f(t)$ and $t^{2} f(t)$ have a bounded total variation on $[0, \infty)$ since we have assumed that $t \mapsto \int_{0}^{t}|d \varphi(s)|$ has at most polynomial growth. Thus, Feller [13], Theorem 4, tells us that $u(t) \rightarrow 1 / b_{1}$ as $t \rightarrow \infty$, which gives $\Gamma(t) \sim e^{\alpha_{0} t} / b_{1}$. This completes the proof, since $1 / b_{1}=a_{0} \alpha_{0}^{2} / \mu$ by definition of $a_{0}$.

Step 4. We now conclude the proof of (a) and (b). Recall that $m_{t}=\mu t+$ $\int_{0}^{t} \int_{0}^{s} \varphi(s-u) m_{u}^{\prime} d u d s$, so that $m^{\prime}=\mu+\varphi \star m^{\prime}$. Applying (e), we deduce that $m^{\prime}=\mu+\mu \star \Gamma=\mu(1+\Upsilon)$. By step 3, we know that $\Gamma(t) \sim\left(a_{0} \alpha_{0}^{2} / \mu\right) e^{\alpha_{0} t}$ as $t \rightarrow \infty$. This obviously implies that $\Upsilon(t) \sim\left(a_{0} \alpha_{0} / \mu\right) e^{\alpha_{0} t}$, whence $m_{t}^{\prime} \sim a_{0} \alpha_{0} e^{\alpha_{0} t}$ and finally $m_{t}=\int_{0}^{t} m_{s}^{\prime} d s \sim a_{0} e^{\alpha_{0} t}$. Finally, (b) directly follows from (a) and the facts that $\Gamma, \Upsilon, m$ and $m^{\prime}$ are nonnegative and locally bounded.

Step 5. Point (d) is not very difficult: using that $\varphi(t) \leq C(1+t)^{p}$, we see that $\varphi^{\star n}(t) \leq C_{n}(1+t)^{p_{n}}$ for some constants $C_{n}>0$ and $p_{n}>0$. We next introduce $\varepsilon_{k}=\sup _{n \geq k}\left|\eta_{n}\right|$, which decreases to 0 as $k \rightarrow \infty$, and write, for any $k \geq 1$,

$$
\begin{aligned}
& \limsup _{t \rightarrow \infty} e^{-\alpha_{0} t} \sum_{n \geq 1}\left|\eta_{n}\right| \int_{0}^{t} \varphi^{\star n}(t-s) e^{\alpha_{0} s / 2} d s \\
& \leq \limsup _{t \rightarrow \infty} e^{-\alpha_{0} t} \sum_{n=1}^{k}\left|\eta_{n}\right| \int_{0}^{t} C_{n}(t-s)^{p_{n}} e^{\alpha_{0} s / 2} d s \\
& \quad+\varepsilon_{k} \limsup _{t \rightarrow \infty} e^{-\alpha_{0} t} \sum_{n=k+1}^{\infty} \int_{0}^{t} \varphi^{\star n}(t-s) e^{\alpha_{0} s / 2} d s .
\end{aligned}
$$

The first term on the RHS is of course 0 (for any fixed $k$ ). We can bound the second one, using (b), by

$$
\begin{aligned}
& \varepsilon_{k} \limsup _{t \rightarrow \infty} e^{-\alpha_{0} t} \int_{0}^{t} \Gamma(t-s) e^{\alpha_{0} s / 2} d s \\
& \quad \leq C \varepsilon_{k} \limsup _{t \rightarrow \infty} e^{-\alpha_{0} t} \int_{0}^{t} e^{\alpha_{0}(t-s)} e^{\alpha_{0} s / 2} d s \leq C \varepsilon_{k} .
\end{aligned}
$$

Letting $k$ tend to infinity completes the proof.

Step 6. It only remains to check point (c). We use the Lebesgue dominated convergence theorem. Define $h_{s}^{t}=\left(\Upsilon(t-s) / m_{t}-\alpha_{0} e^{-\alpha_{0} s} / \mu\right)^{2} m_{s}^{\prime} \mathbf{1}_{\{s \leq t\}}$. We have to 
prove that $\lim _{t \rightarrow \infty} \int_{0}^{\infty} h_{s}^{t} d s=0$. First, it is obvious from (a) that for $s>0$ fixed, $\lim _{t \rightarrow \infty} h_{s}^{t}=0$. Next, we use (b) to write (for $t$ large enough so that $m_{t} \geq c e^{\alpha_{0} t}$ ) $h_{s}^{t} \leq C\left(e^{-\alpha_{0} s}\right)^{2} e^{\alpha_{0} s} \leq C e^{-\alpha_{0} s}$, which does not depend on $t$ and is integrable on $(0, \infty)$.

We next consider briefly a vector convolution equation.

LEMMA 27. Consider a family $\boldsymbol{\mu}=\left(\mu_{i}\right)_{i \in \mathbb{Z}^{d}}$ of real numbers such that $0 \leq$ $\mu_{i} \leq C$ for all $i \in \mathbb{Z}^{d}$, the stochastic matrix $(A(i, j))_{i, j \in \mathbb{Z}^{d}}$ defined by $(17)$, and a locally integrable function $\varphi:(0, \infty) \mapsto[0, \infty)$. The equation $\mathbf{m}_{t}=\boldsymbol{\mu} t+\int_{0}^{t} \varphi(t-$ $s)$ A $\mathbf{m}_{s} d s$ with unknown $\mathbf{m}=\left(m_{t}^{i}\right)_{t \geq 0, i \in \mathbb{Z}^{d}}$ has a unique solution such that for all $t \geq 0, \sum_{i \in \mathbb{Z}^{d}} 2^{-|i|} \sup _{[0, t]} m_{s}^{i}<\infty$. Furthermore, $m^{i}$ is of class $C^{1}$ on $[0, \infty)$ for all $i \in \mathbb{Z}^{d}$, and it holds that $\mathbf{m}_{t}^{\prime}=\boldsymbol{\mu}+\int_{0}^{t} \varphi(t-s) A \mathbf{m}_{s}^{\prime} d s$ and $\mathbf{m}_{t}^{\prime}=(I+$ $\left.\sum_{n \geq 1} A^{n} \int_{0}^{t} \varphi^{\star n}(s) d s\right) \mu$. Finally, $u_{t}=\sup _{i \in \mathbb{Z}^{d}} \sup _{[0, t]}\left(m_{s}^{i}\right)^{\prime}$ is finite for all $t \geq 0$ and it holds that $u_{t} \leq C+\int_{0}^{t} \varphi(t-s) u_{s} d s$.

PROOF. We proceed in a few steps.

Step 1 . We first note that for any vector $\mathbf{x}=\left(x_{i}\right)_{i \in \mathbb{Z}^{d}}$, it holds that $\sum_{i \in \mathbb{Z}^{d}} 2^{-|i|} \times$ $\left|(A \mathbf{x})_{i}\right| \leq 2 \sum_{i \in \mathbb{Z}^{d}} 2^{-|i|}\left|x_{i}\right|$. This easily follows from the explicit form of $A$.

Step 2. We next check uniqueness. Consider two solutions $\mathbf{m}$ and $\tilde{\mathbf{m}}$ satisfying the required condition and put $h_{t}=\sum_{i \in \mathbb{Z}^{d}} 2^{-|i|} \sup _{[0, t]}\left|m_{t}^{i}-\tilde{m}_{t}^{i}\right|$. We have $h_{t} \leq \sum_{i \in \mathbb{Z}^{d}} 2^{-|i|} \int_{0}^{t} \varphi(t-s)\left|A\left(\mathbf{m}_{s}-\tilde{\mathbf{m}}_{s}\right)_{i}\right| d s$. Using step 1, we deduce that $h_{t} \leq 2 \int_{0}^{t} \varphi(t-s) h_{s} d s$ and thus $h_{t}=0$ by Lemma 23(i).

Step 3. We define $\mathbf{m}_{t}^{\prime}=\left(I+\sum_{n \geq 1} A^{n} \int_{0}^{t} \varphi^{\star n}(s) d s\right) \mu$. Using that $A$ is stochastic and that $\boldsymbol{\mu}$ is bounded (by $C$ ), we easily deduce that for all $i \in \mathbb{Z}^{d},\left(m_{t}^{i}\right)^{\prime} \leq$ $C\left(1+\sum_{n \geq 1} \int_{0}^{t} \varphi^{\star n}(s) d s\right)$. This function is locally bounded because $\varphi$ is locally integrable: use that $\Upsilon_{k}(t)=\sum_{n=1}^{k} \int_{0}^{t} \varphi^{\star n}(s) d s$ satisfies $\Upsilon_{k}(t) \leq \int_{0}^{t} \varphi(s) d s+$ $\int_{0}^{t} \varphi(t-s) \Upsilon_{k}(s) d s$ and use Lemma 23(iii), which provides a uniform (in $k$ ) bound. Consequently, $u_{t}=\sup _{i \in \mathbb{Z}^{d}} \sup _{[0, t]}\left(m_{s}^{i}\right)^{\prime}$ is finite for all $t \geq 0$. Similar arguments show that $\left(m_{t}^{i}\right)^{\prime}$ is continuous on $[0, \infty)$, because $\left|\left(m_{t+h}^{i}\right)^{\prime}-\left(m_{t}^{i}\right)\right| \leq$ $C \sum_{n \geq 1} \int_{t}^{t+h} \varphi^{\star n}(s) d s$.

Step 4. A straightforward consequence of the definition of $\mathbf{m}_{t}^{\prime}$ is that it solves $\mathbf{m}_{t}^{\prime}=\boldsymbol{\mu}+\int_{0}^{t} \varphi(t-s) A \mathbf{m}_{s}^{\prime} d s$. Using that $A$ is stochastic and that $\boldsymbol{\mu}$ is bounded, we immediately deduce that $u_{t} \leq C+\int_{0}^{t} \varphi(t-s) u_{s} d s$. We finally define, for each $i \in$ $\mathbb{Z}^{d}, m_{t}^{i}=\int_{0}^{t}\left(m_{s}^{i}\right)^{\prime} d s$. Integrating the equation satisfied by $\mathbf{m}^{\prime}$ and using Lemma 22, we find that $\mathbf{m}=\left(m_{t}^{i}\right)_{i \in \mathbb{Z}^{d}, t \geq 0}$ is indeed a solution to $\mathbf{m}_{t}=\boldsymbol{\mu} t+\int_{0}^{t} \varphi(t-s) A \mathbf{m}_{s} d s$. It only remains to check that $\sum_{i \in \mathbb{Z}^{d}} 2^{-|i|} \sup _{[0, t]} m_{t}^{i}<\infty$ for all $t \geq 0$, but this obviously follows from the facts that $u_{t}$ is locally bounded and that $\mathbf{m}_{0}=0$. 


\section{REFERENCES}

[1] Aït-Sahalia, Y., Cacho-Diaz, J. and Laeven, R. J. A. (2015). Modeling financial contagion using mutually exciting jump processes. J. Financial Econ. 117 585-606.

[2] Bacry, E., Delattre, S., Hoffmann, M. and Muzy, J. F. (2013). Modelling microstructure noise with mutually exciting point processes. Quant. Finance 13 65-77. MR3005350

[3] Bacry, E., Delattre, S., Hoffmann, M. and Muzy, J. F. (2013). Some limit theorems for Hawkes processes and application to financial statistics. Stochastic Process. Appl. 123 2475-2499. MR3054533

[4] BACRY, E. and MuZY, J. F. Second order statistics characterization of Hawkes processes and non-parametric estimation. Available at arXiv:1401.0903.

[5] BACRY, E. and MUZY, J.-F. (2014). Hawkes model for price and trades high-frequency dynamics. Quant. Finance 14 1147-1166. MR3219705

[6] BaUwens, L. and HaUtsch, N. (2009). Modelling financial high frequency data using point processes. In Handbook of Financial Time Series (T. Mikosch, J.-P. Kreiss, R. A. Davis and T. G. Andersen, eds.). Springer, Berlin.

[7] Blundell, C., Heller, K. A. and BeCK, J. F. (2012). Modelling reciprocating relationships with Hawkes processes. In Neural Information Processing Systems (NIPS).

[8] Bordenave, C. and Torrisi, G. L. (2007). Large deviations of Poisson cluster processes. Stoch. Models 23 593-625. MR2362700

[9] Brémaud, P. and Massoulié, L. (1996). Stability of nonlinear Hawkes processes. Ann. Probab. 24 1563-1588. MR1411506

[10] BRÉmaUd, P., NAPPO, G. and TORRISI, G. L. (2002). Rate of convergence to equilibrium of marked Hawkes processes. J. Appl. Probab. 39 123-136. MR1895148

[11] Chevallier, J. (2013). Détection de motifs de dépendance entre neurones. Preprint.

[12] Daley, D. J. and Vere-Jones, D. (2003). An Introduction to the Theory of Point Processes. Vol. I: Elementary Theory and Methods, 2nd ed. Springer, New York. MR1950431

[13] Feller, W. (1941). On the integral equation of renewal theory. Ann. Math. Stat. 12 243-267. MR0005419

[14] Fournier, N. and Guillin, A. On the rate of convergence in Wasserstein distance of the empirical measure. Available at arXiv:1312.2128.

[15] Galves, A. and LÖCHERBACH, E. (2013). Infinite systems of interacting chains with memory of variable length—a stochastic model for biological neural nets. J. Stat. Phys. 151896 921. MR3055382

[16] GRIgelionis, B. (1971). The representation of integer-valued random measures as stochastic integrals over the Poisson measure. Litovsk. Mat. Sb. 11 93-108. MR0293703

[17] GrÜn, S., Diedsmann, M. and Aertsen, A. M. (2010). Unitary events analysis. In Analysis of Parallel Spike Trains (S. Grün and S. Rotter, eds.) 191-220. Springer, Berlin.

[18] Hansen, N. R., Reynaud-Bouret, P. and Rivoirard, V. (2015). Lasso and probabilistic inequalities for multivariate point processes. Bernoulli 21 83-143.

[19] Hawkes, A. G. (1971). Spectra of some self-exciting and mutually exciting point processes. Biometrika 58 83-90. MR0278410

[20] Hawkes, A. G. and OAKes, D. (1974). A cluster process representation of a self-exciting process. J. Appl. Probab. 11 493-503. MR0378093

[21] Helmstetter, A. and Sornette, D. (2002). Subcritical and supercritical regimes in epidemic models of earthquake aftershocks. J. Geophys. Res. 1072237.

[22] Hewlett, P. (2006). Clustering of order arrivals, price impact and trade path optimisation. In Workshop on Financial Modeling with Jump Processes. Ecole Polytechnique.

[23] JaCOD, J. (1979). Calcul Stochastique et Problèmes de Martingales. Lecture Notes in Math. 714. Springer, Berlin. MR0542115 
[24] Jacod, J. and ShiRYAev, A. N. (2003). Limit Theorems for Stochastic Processes, 2nd ed. Grundlehren der Mathematischen Wissenschaften [Fundamental Principles of Mathematical Sciences] 288. Springer, Berlin. MR1943877

[25] Jaisson, T. and Rosenbaum, M. Limit theorems for nearly unstable Hawkes processes. Ann. Appl. Probab. To appear. Available at arXiv:1310.2033.

[26] John, F. (1991). Partial Differential Equations, 4th ed. Applied Mathematical Sciences 1. Springer, New York. MR1185075

[27] Kagan, Y. Y. (2010). Statistical distributions of earthquake numers: Consequence of branching process. Geophys. J. Int. $1801313-1328$.

[28] Kerstan, J. (1964). Teilprozesse Poissonscher Prozesse. In Trans. Third Prague Conf. Information Theory, Statist. Decision Functions, Random Processes (Liblice, 1962) 377-403. Publ. House Czech. Acad. Sci., Prague. MR0166826

[29] LaWler, G. F. and Limic, V. (2010). Random Walk: A Modern Introduction. Cambridge Studies in Advanced Mathematics 123. Cambridge Univ. Press, Cambridge. MR2677157

[30] LEWIS, P. A. W. (1969). Asymptotic properties and equilibrium conditions for branching Poisson processes. J. Appl. Probab. 6 355-371. MR0254931

[31] Lewis, P. A. W. and SHEdLER, G. S. (1979). Simulation of nonhomogeneous Poisson processes by thinning. Nav. Res. Logist. Q. 26 403-413. MR0546120

[32] Massoulié, L. (1998). Stability results for a general class of interacting point processes dynamics, and applications. Stochastic Process. Appl. 75 1-30. MR1629010

[33] OAKes, D. (1975). The Markovian self-exciting process. J. Appl. Probab. 12 69-77. MR0362522

[34] OGatA, Y. (1978). The asymptotic behaviour of maximum likelihood estimators for stationary point processes. Ann. Inst. Statist. Math. 30 243-261. MR0514494

[35] OgatA, Y. (1981). On Lewis simulation method for point processes. IEEE Trans. Inform. Theory 27 23-30.

[36] Ogata, Y. (1999). Seismicity analysis through point-process modeling: A review. Pure Appl. Geophys. 155 471-507.

[37] Okatan, M., Wilson, M. A. and Brown, E. N. (2005). Analyzing functional connectivity using a network likelihood model of ensemble neural spiking activity. Neural Comput. 17 1927-1961.

[38] Pillow, J. W., Shlens, J., Paninski, L., Scher, A., Litke, A. M., Chichilnisky, E. J. and SimoncelLI, E. P. (2008). Spatio-temporal correlations and visual signalling in a complete neuronal population. Nature 454 995-999.

[39] Reynaud-Bouret, P., Rivoirard, V., Grammont, F. and Tuleau-Malot, C. (2014). Goodness-of-fit tests and nonparametric adaptive estimation for spike train analysis. J. Math. Neurosci. 4 Art. 3, 41. MR3197017

[40] Reynaud-Bouret, P., Rivoirard, V. and Tuleau-Malot, C. (2013). Inference of functional connectivity in neurosciences via Hawkes processes. In 1st IEEE Global Conference on Signal and Information Processing.

[41] Reynaud-Bouret, P. and Schbath, S. (2010). Adaptive estimation for Hawkes processes; application to genome analysis. Ann. Statist. 38 2781-2822. MR2722456

[42] Simma, A. and Jordan, M. I. Modeling events with cascades of Poisson processes. Available at http://www.eecs.berkeley.edu/Pubs/TechRpts/2010/EECS-2010-109.html.

[43] Sznitman, A.-S. (1991). Topics in propagation of chaos. In École D'Été de Probabilités de Saint-Flour XIX-1989. Lecture Notes in Math. 1464 165-251. Springer, Berlin. MR1108185

[44] Torrisi, G. L. and LeONARdi, E. (2013). Simulating the tail of the interference in a Poisson network model. IEEE Trans. Inform. Theory 59 1773-1787. MR3030752

[45] YANG, S.-H. and ZHA, H. (2013). Mixture of mutually exciting processes for viral diffusion. In Proceedings of the 30th International Conf. on Machine Learning (ICML). 
[46] ZHOU, K., ZHA, H. and SoNG, L. (2013). Learning triggering kernels for multi-dimensional Hawkes processes. In Proceedings of the 30th International Conf. on Machine Learning $(I C M L)$.

[47] ZHU, L. (2013). Central limit theorem for nonlinear Hawkes processes. J. Appl. Probab. 50 760-771. MR3102513

[48] ZHU, L. (2013). Nonlinear Hawkes processes. Ph.D. thesis, New York Univ. Available at arXiv:1304.7531.

[49] Zhu, L. (2015). Large deviations for Markovian nonlinear Hawkes processes. Ann. Appl. Probab. 25 548-581. MR3313748

\section{S. DELATTRE}

LABORATOIRE DE Probabilités ET MOdÈLES AlÉATOIRES, UMR 7599

UNIVERSITÉ PARIS-DIDEROT

CASE COURRIER 7012

AVENUE DE FRANCE

75205 PARIS CEDEX 13

FRANCE

E-MAIL: sylvain.delattre@univ-paris-diderot.fr

\author{
N. FOURNIER \\ LABoratoire DE PRoBABILITÉS ET \\ MOdÈLES AlÉATOIRES, UMR 7599 \\ UNIVERSITÉ PIERRE-ET-MARIE CURIE \\ CASE 188, 4 PLACE JUSSIEU \\ F-75252 PARIS CEDEX 5 \\ FRANCE \\ E-MAIL: nicolas.fournier@upmc.fr
}

\author{
M. HOFFMANN \\ CEREMADE, CNRS-UMR 7534 \\ UNIVERSITÉ PARIS-DAUPHINE \\ Place du Maréchal De LatTre de TASSigny \\ 75775 PARIS CEDEX 16 \\ FRANCE \\ E-MAIL: hoffmann@ceremade.dauphine.fr
}

